\title{
Identification of potential mRNA panels for severe acute respiratory syndrome coronavirus 2 (COVID-19) diagnosis and treatment using microarray dataset and bioinformatics methods
}

\author{
Basavaraj Vastrad $^{1}$ (D) . Chanabasayya Vastrad ${ }^{2}$ D . Anandkumar Tengli ${ }^{3}$ (D)
}

Received: 21 May 2020 / Accepted: 20 August 2020 / Published online: 11 September 2020

(c) King Abdulaziz City for Science and Technology 2020

\begin{abstract}
The goal of the present investigation is to identify the differentially expressed genes (DEGs) between SARS-CoV-2 infected and normal control samples to investigate the molecular mechanisms of infection with SARS-CoV-2. The microarray data of the dataset E-MTAB-8871 were retrieved from the ArrayExpress database. Pathway and Gene Ontology (GO) enrichment study, protein-protein interaction (PPI) network, modules, target gene-miRNA regulatory network, and target gene-TF regulatory network have been performed. Subsequently, the key genes were validated using an analysis of the receiver operating characteristic (ROC) curve. In SARS-CoV-2 infection, a total of 324 DEGs (76 up- and 248 down-regulated genes) were identified and enriched in a number of associated SARS-CoV-2 infection pathways and GO terms. Hub and target genes such as TP53, HRAS, MAPK11, RELA, IKZF3, IFNAR2, SKI, TNFRSF13C, JAK1, TRAF6, KLRF2, CD1A were identified from PPI network, target gene-miRNA regulatory network, and target gene-TF regulatory network. Study of the ROC showed that ten genes (CCL5, IFNAR2, JAK2, MX1, STAT1, BID, CD55, CD80, HAL-B, and HLA-DMA) were substantially involved in SARS-CoV-2 patients. The present investigation identified key genes and pathways that deepen our understanding of the molecular mechanisms of SARS-CoV-2 infection, and could be used for SARS-CoV-2 infection as diagnostic and therapeutic biomarkers.
\end{abstract}

Keywords SARS-CoV-2 infection · Bioinformatics analysis · Biomarkers · Protein-protein interaction (PPI) network · Differentially expressed genes

\section{Introduction}

Severe acute respiratory syndrome coronavirus 2 (SARS$\mathrm{CoV}-2)$ infection is known as novel coronavirus disease-2019 (COVID-19) and has spread widely throughout the globe in an epidemic proportion with the current pandemic risk ( $\mathrm{Li}$ et al. 2020). This infection is related to respiratory diseases, and this virus mainly infects respiratory

Chanabasayya Vastrad

channu.vastrad@gmail.com

1 Department of Pharmaceutics, SET'S College of Pharmacy, Dharwad, Karnataka 580002, India

2 Biostatistics and Bioinformatics, Chanabasava Nilaya, Bharthinagar, Dharwad, Karnataka 580001, India

3 Department of Pharmaceutical Chemistry, JSS College of Pharmacy, Mysuru and JSS Academy of Higher Education \& Research, Mysuru, Karnataka 570015, India epithelial cells and transmits from human to human primarily through the respiratory tract, contributing to more deaths (Zou et al. 2020; Madurai Elavarasan and Pugazhendhi 2020). In the present situation, the survival rate of patients with SARS-CoV-2 infection has been slightly increased, and patients with this infection have no apparent benefit from the current antiviral drugs (Hoffmann et al. 2020). Knowing the molecular pathogenesis of the viral infections and their routes of transmission is completely necessary for the creation of new therapeutic targets.

Present situation for investigating the pathogenesis of SARS-CoV-2 infection is needed in molecular biology. Although the pathogenesis of SARS-CoV-2 infection remains to be clarified, abnormal gene expression in nasal epithelial cells can serve significant roles (Sungnak et al. 2020). Entry factors related genes such as angiotensin-converting enzyme 2 (ACE2) (Zhang et al. 2020); TMPRSS2 (Sungnak et al. 2020); and inflammatory related genes (IL-2, IL-7, IL-10, GCSF, IP-10, MCP-1, MIP-1A, and TNF- $\alpha$ ) (Fu 
et al. 2020) were linked with pathogenesis of SARS-CoV-2 infections. Therefore, targeted regulation of these genes may reveal potential strategies for the treatment of SARS-CoV-2 infections. Therefore, targeted regulation of entry factors and inflammatory-related genes could become potential strategies for the treatment of SARS-CoV-2 infection.

Throughout this investigation, we used bioinformatics methods to examine differentially expressed genes (DEGs) between SARS-CoV-2-infected samples and standard control samples. We performed pathway enrichment and gene ontology (GO) analysis of DEGs, and established the protein-protein interactions (PPI) network, modules analysis, target gene-miRNA regulatory network, and target gene-TF regulatory network to reveal molecular mechanisms in SARS-CoV-2 infection. Finally, we performed validation hub genes by receiver operating characteristic (ROC) curve analysis. Finally, through receiver operating characteristic (ROC) curve analysis, we conducted validation hub genes. The aim of this study is thus to have a better understanding of the exact mechanisms of SARS-CoV-2 infection and to identify potential novel diagnostic or therapeutic targets through bioinformatics analysis.

\section{Materials and methods}

\section{Microarray data selection}

Microarray data of gene expression profile (E-MTAB-8871) was downloaded from ArrayExpress (https://www.ebi. ac.uk/arrayexpress), which is the largest resource of gene expression publicly available (Kolesnikov et al. 2015). Samples from this dataset were RNA extracted from the blood sample and processed for hybridization on NanoString nCounter Human Immunology V2 Panel Array. A total of 32 samples were investigated, including 22 SARS-CoV-2-infected samples, and 10 normal control samples. The study was designed according to the flowchart (Fig. 1).

\section{Identification of DEGs}

The DEGs between the SARS-CoV-2-infected samples and normal control samples were analyzed with various methods including data preparation (data normalization and summarization) and DEGs identification (up- and downregulated genes). The limma package in $\mathrm{R}$ Software was used for background correction, quantile normalization and probe summarization, and limma package was also applied for DEGs identification (Ritchie et al. 2015). The development of DEGs choice included model design, linear model fitness, contrast matrix generation, bayesian model building and gene filtering, all of which were managed by the functions in the limma package. Genes with the $p<0.05$, Ilog $\mathrm{Fcl}$ (fold change) $>1.5$ were considered as DEGs (up- and down-regulated genes).

\section{Pathway enrichment analysis for DEGs}

BIOCYC (https://biocyc.org/) (Caspi et al. 2016), Kyoto Encyclopedia of Genes and Genomes (KEGG) (http://www. genome.jp/kegg/pathway.html) (Kanehisa et al. 2019), Pathway Interaction Database (PID) (https://wiki.nci.nih.gov/
Fig. 1 The workflow representing the methodology and the major outcome of the study. SARS-CoV-2-Severe acute respiratory syndrome coronavirus 2 infection - breast cancer, GO_-gene ontology, miRNAMicroRNA, TF-transcription factor, DEGs-deferential expressed genes

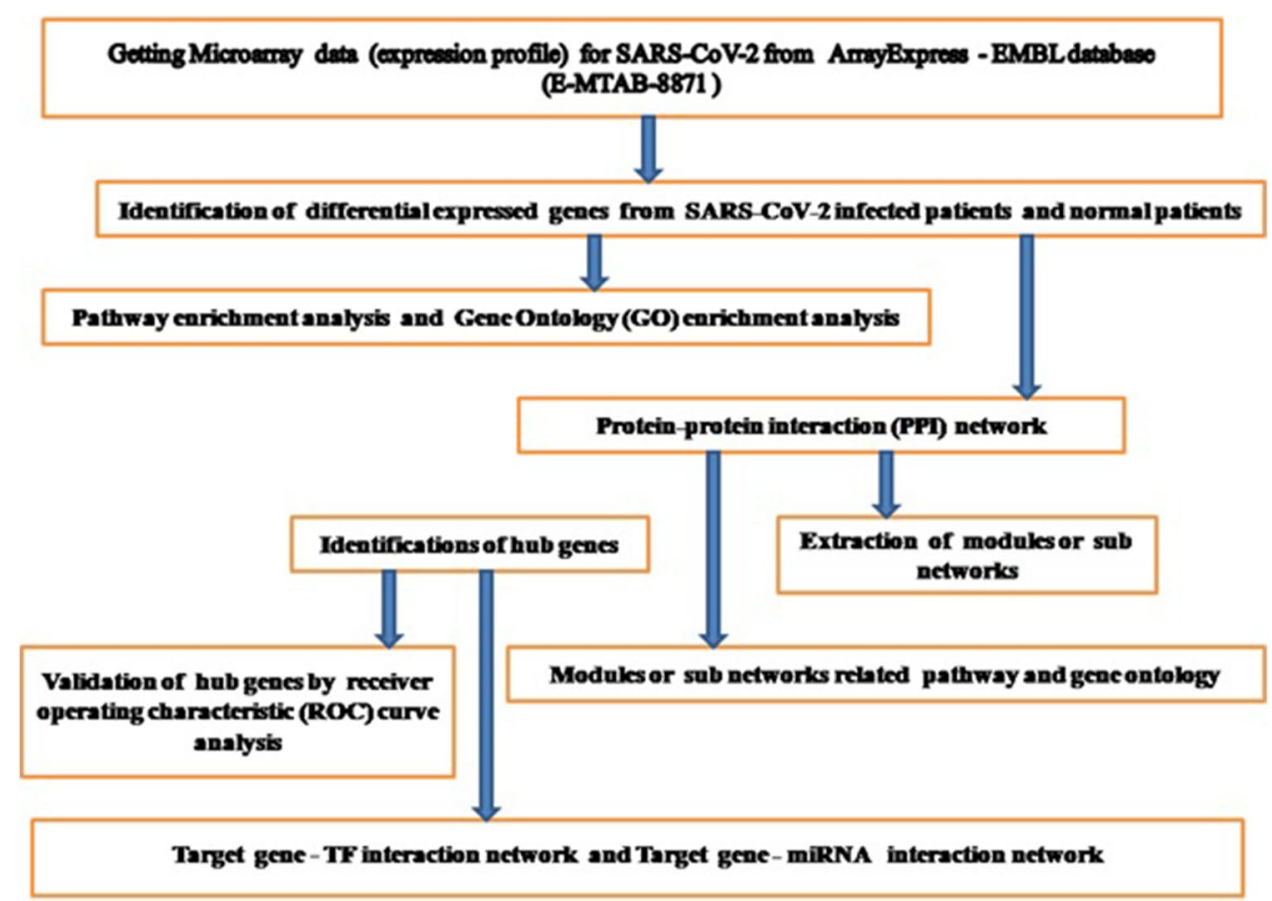


pages/viewpage.action?pageId=315491760) (Schaefer et al. 2009), REACTOME (https://reactome.org/) (Fabregat et al. 2018), GenMAPP (http://www.genmapp.org/) (Dahlquist et al. 2002), MSigDB C2 BIOCARTA (http://software.broad institute.org/gsea/msigdb/collections.jsp) (Subramanian et al. 2005), PantherDB (http://www.pantherdb.org/) (Mi et al. 2017), Pathway Ontology (http://www.obofoundry.org/ ontology/pw.html) (Petri et al. 2014) and Small Molecule Pathway Database (SMPDB) (http://smpdb.ca/) (Jewison et al. 2014) are a data resource for genes and genomes with assigned corresponding functional importance. The ToppGene (ToppFun) (https://toppgene.cchmc.org/enrichment .jsp) (Chen et al. 2009) is an online resource for interpreting genes originating from genomic investigation with bioinformatics data. The $p$ value $<0.05$ was considered statistically significant.

\section{Gene ontology (GO) enrichment analysis for DEGs}

GO (http://www.geneontology.org/) (Lewis et al. 2017) was used to determine gene actions in three aspects: biological process (BP), cellular component (CC) and molecular function (MF). ToppGene (ToppFun) (https://toppgene.cchmc .org/enrichment.jsp) (Chen et al. 2009) is an online website that provides an extensive set of functional annotation tools to understand the biological meaning behind a massive list of genes. In the current investigation, the $\mathrm{GO}$ enrichment analyses for statistically important DEGs. The $p$ value $<0.05$ was considered statistically significant.

\section{PPI network construction and module analysis}

The common up and down-regulated genes of E-MTAB-8871 was analyzed using the online website STRING (https://string-db.org/, version 11) (Szklarczyk et al. 2019), with 0.700 (moderate confidence) as the minimum required interaction score. Then, the software Cytoscape (http://www.cytoscape.org/, version 3.8.0) (Shannon et al. 2003) was used to establish a PPI network. The Network Analyzer in Cytoscape was utilized to calculate node degree (Przulj et al. 2004), betweenness centrality (Nguyen et al. 2011), stress centrality (Shi and Zhang 2011), closeness centrality (Nguyen and Liu 2011) and clustering coefficient (Wang et al. 2012). PEWCC1 (http://apps.cytos cape.org/apps/PEWCC1) (Zaki et al. 2013) was used to perform module analysis.

\section{Construction of target gene-miRNA regulatory network}

miRNet database (https://www.mirnet.ca/) (Fan and Xia 2018) provides certain target gene-miRNA regulatory association pairs, which are verified by experiments and predicted by ten programs, including TarBase (http://diana .imis.athena-innovation.gr/DianaTools/index.php? $\mathrm{r}=$ tarba se/index) (Vlachos et al. 2015), miRTarBase (http://mirta rbase.mbc.nctu.edu.tw/php/download.php) (Chou et al. 2018), miRecords (http://miRecords.umn.edu/miRecords) (Xiao et al. 2009), miR2Disease (http://www.mir2diseas e.org/) (Jiang et al. 2009), HMDD (http://www.cuilab.cn/ hmdd) (Huang et al. 2019), PhenomiR (http://mips.helmh oltz-muenchen.de/phenomir/) (Ruepp et al. 2010), SM2miR (http://bioinfo.hrbmu.edu.cn/SM2miR/) (Liu et al. 2013), PharmacomiR (http://www.pharmaco-mir.org/) (Rukov et al. 2014), EpimiR (http://bioinfo.hrbmu.edu.cn/EpimiR/) (Dai et al. 2014) and starBase (http://starbase.sysu.edu.cn/) (Li et al. 2014). This investigation inputted the up- and downregulated genes into the database to examine the regulatory association pairs between target gene and miRNA. Target gene-miRNA regulatory network was constructed and visualized by Cytoscape 3.8.0 software to show the target genes and miRNA. Therefore, these target genes and miRNA might play a potential role in the pathogenesis and treatment of SARS-CoV-2 infection.

\section{Construction of target gene-TF regulatory network}

NetworkAnalyst database (https://www.networkanalyst.ca/) (Zhou et al. 2019) provides certain target gene-TF regulatory association pairs, which are verified by experiments and predicted by JASPAR (http://jaspar.genereg.net/) (Khan et al. 2018) database. This investigation inputted the up- and down-regulated genes into the database to examine the regulatory association pairs between target gene and TF. Target gene-TF regulatory network was constructed and visualized by Cytoscape 3.8.0 software to show the target genes and TF. Therefore, these target genes and TF may play a potential role in the pathogenesis and treatment of SARS-CoV-2 infection.

\section{Validation of hub genes}

Receiver-operating characteristic (ROC) analyses were operated to calculate the diagnostic value of the hub genes for SARS-CoV-2 infection. The ROC curve with area under curve (AUC) was determined using R "pROC" package (Robin et al. 2011).

\section{Results}

\section{Identification of DEGs}

Microarray dataset (E-MTAB-8871) was obtained from ArrayExpress database and normalized mRNA expression data through R language (Fig. 2). Volcano plot was

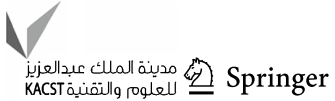



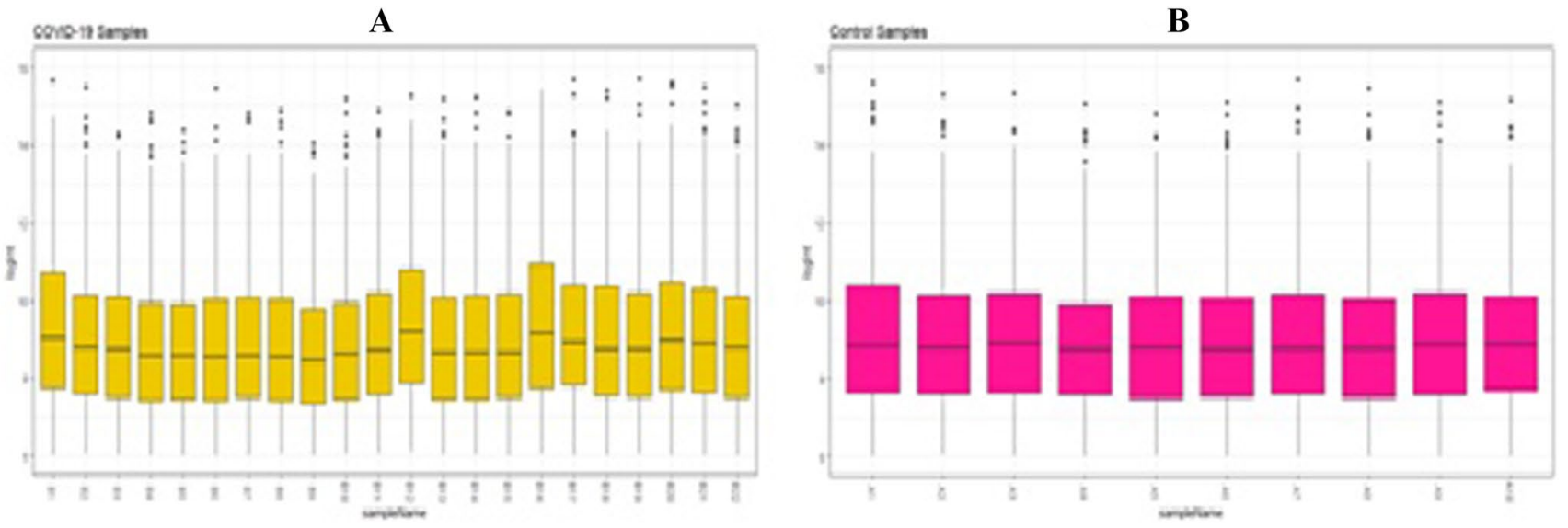

$\square$ =SARS-CoV-2 infected samples $\square$ = Normal control samples

Fig. 2 Box plots of the normalized data. a 22 SARS-CoV-2 infected samples b 10 normal control samples. Horizontal axis represents the sample symbol and the vertical axis represents the gene expression values. The black line in the box plot represents the median value of gene expression

generated to manifest up-regulated (green) and down-regulated (red) genes between SARS-CoV-2-infected samples and normal controls samples (Fig. 3) and were also visualized on a heatmap for up- and down-regulated genes (Figs. 4, 5). This approach indicated presence of a total of 324 statistically significant genes $(P<0.05, \log \mathrm{Fcl}$ (fold change) $>1.5$ ), of which 76 genes were up-regulated and 248 genes were down-regulated (Table 1).

\section{Pathway enrichment analysis for DEGs}

Pathway enrichment analysis of integrated DEGs showed the up-regulated genes were mainly involved in measles, herpes simplex infection, IL12-mediated signaling events, IL2mediated signaling events, cytokine signaling in immune system, innate immune system, IL22 soluble receptor signaling pathway, bioactive peptide-induced signaling pathway, JAK/STAT signaling pathway, Inflammation mediated by chemokine and cytokine signaling pathway, $G$ protein signaling, platelet-derived growth factor signaling, intracellular signalling through adenosine receptor A2a and adenosine,

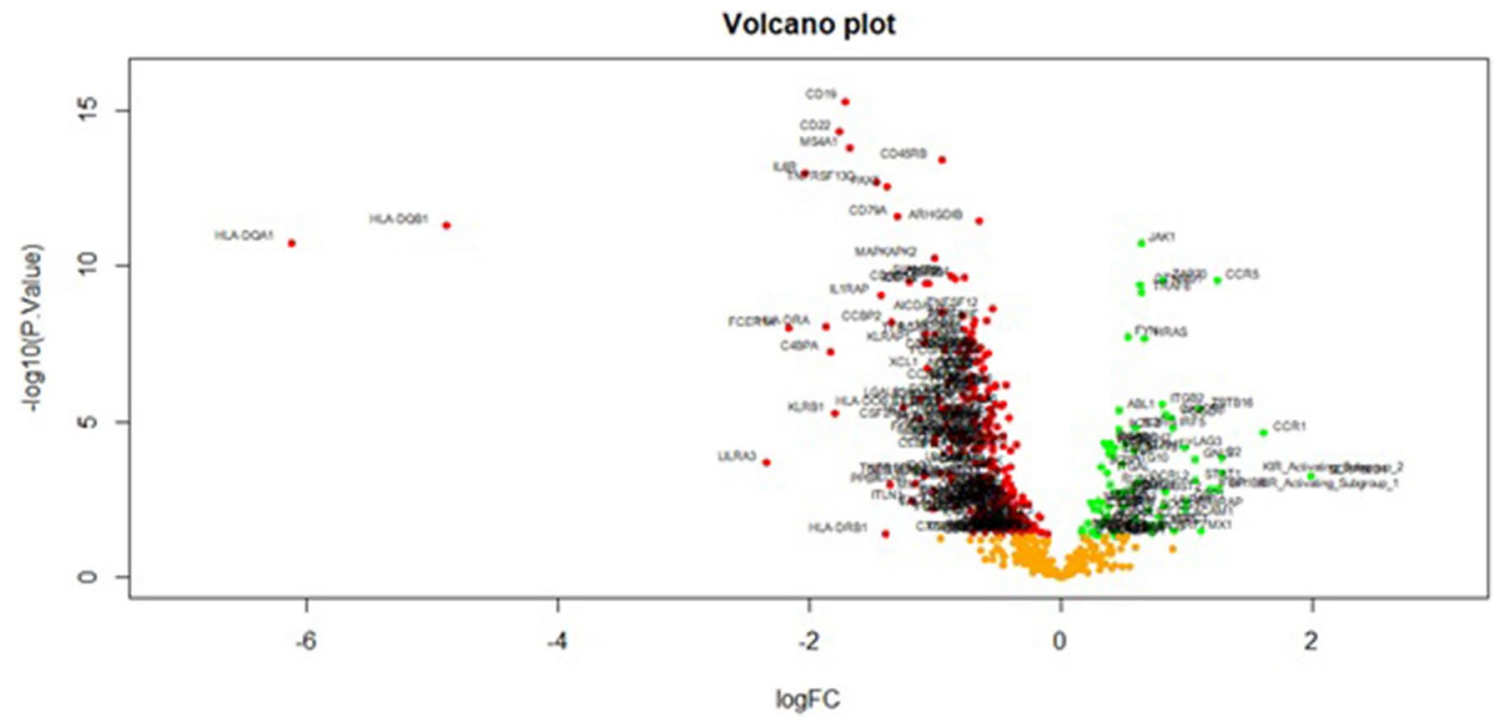

Fig. 3 Volcano plot of differentially expressed genes. Genes with a significant change of more than twofold were selected. Green dot on right side $(\square)$ represented up regulated significant genes and red dot on left side ( ) represented down regulated significant genes 

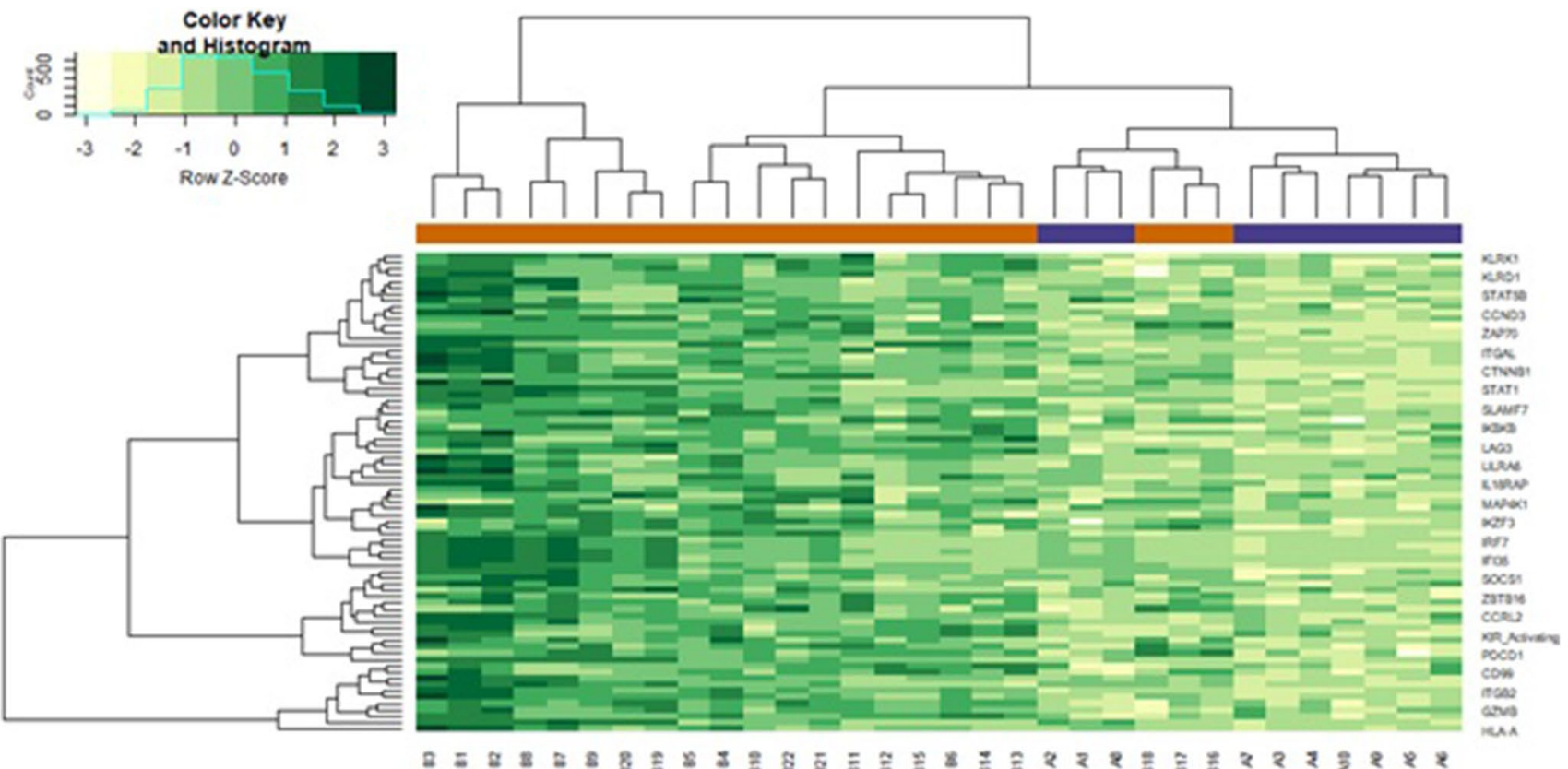

Fig. 4 Heat map of up regulated differentially expressed genes. Legend on the top left indicate log fold change of genes. White represents decreased expression of genes; light green represents not significant expression of genes; dark green represents increased expression of genes. $(\mathrm{A} 1-\mathrm{A} 10=$ Normal control samples; B1-B22 $=$ SARS-CoV-2 infected samples)
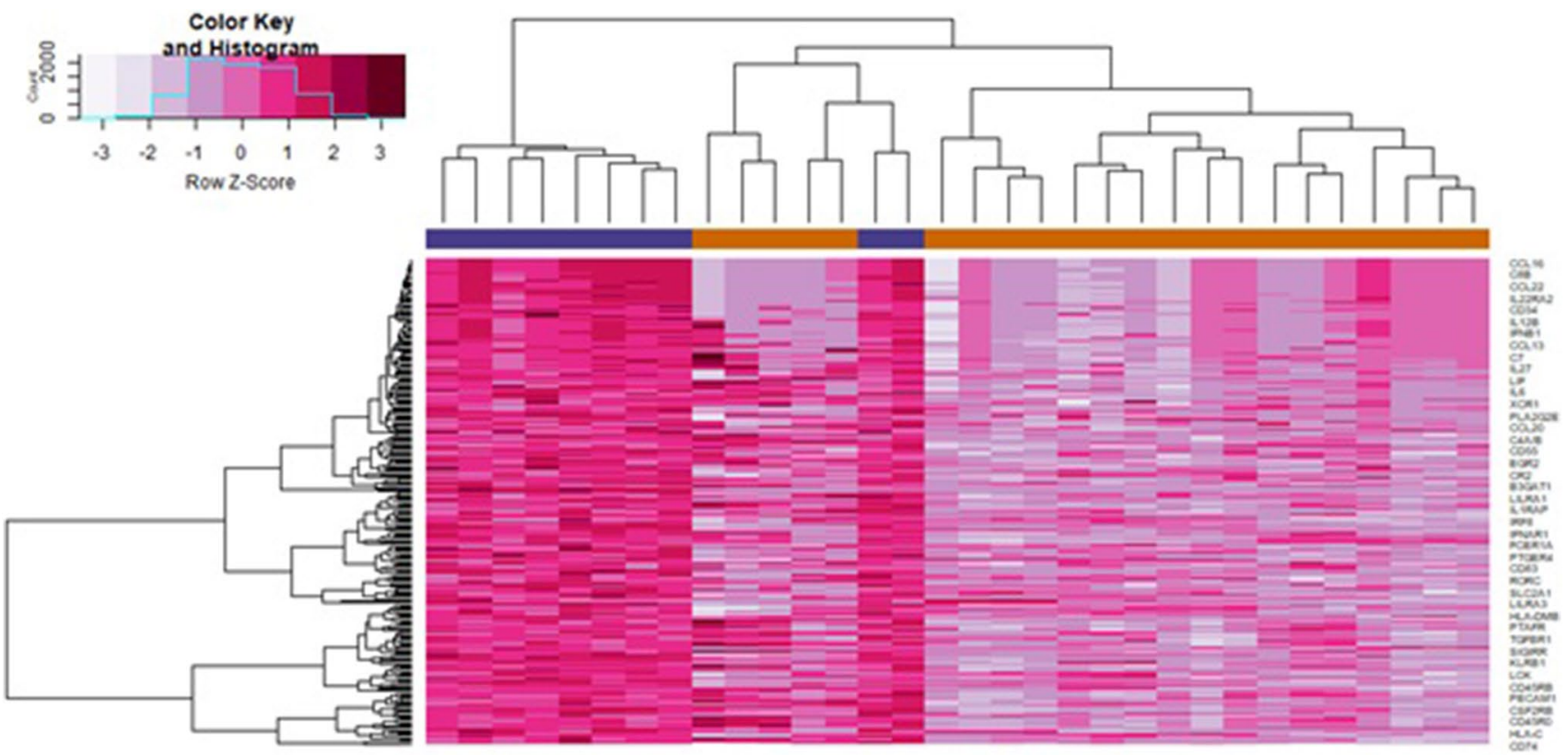

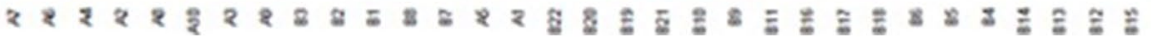

Fig. 5 Heat map of down regulated differentially expressed genes. Legend on the top left indicate log fold change of genes. White represents decreased expression of genes; light pink represents not signifi-

insulin signalling and other pathways (Table 2); the downregulated genes were mainly involved in citrulline-nitric oxide cycle, phospholipases, cytokine-cytokine receptor cant expression of genes; dark pink represents increased expression of genes. $(\mathrm{A} 1-\mathrm{A} 10=$ Normal control samples; B1-B22 $=$ SARS-CoV-2 infected samples)

interaction, hematopoietic cell lineage, IL4-mediated signaling events, IL12-mediated signaling events, cytokine signaling in immune system, signaling by interleukins, 
Table 1 The statistical metrics for key differentially expressed genes (DEGs)

\begin{tabular}{|c|c|c|c|c|c|c|}
\hline Gene Symbol & $\log \mathrm{FC}$ & pValue & adj.P.Val & $\mathrm{t}$ value & Regulation & Gene Name \\
\hline JAK1 & 0.641818 & $1.84 \mathrm{E}-11$ & $1.84 \mathrm{E}-11$ & 9.973193 & Up & Janus kinase 1 \\
\hline ZAP70 & 0.810545 & $2.91 \mathrm{E}-10$ & $2.91 \mathrm{E}-10$ & 8.897328 & Up & $\begin{array}{l}\text { Zeta chain of } T \text { cell receptor associated } \\
\text { protein kinase } 70\end{array}$ \\
\hline CCR5 & 1.239182 & $3.04 \mathrm{E}-10$ & $3.04 \mathrm{E}-10$ & 8.880753 & Up & $\begin{array}{l}\mathrm{C}-\mathrm{C} \text { motif chemokine receptor } 5 \text { (gene/ } \\
\text { pseudogene) }\end{array}$ \\
\hline CTNNB1 & 0.633545 & $4.11 \mathrm{E}-10$ & $4.11 \mathrm{E}-10$ & 8.766907 & Up & Catenin beta 1 \\
\hline TRAF6 & 0.644773 & $7.11 \mathrm{E}-10$ & $7.11 \mathrm{E}-10$ & 8.561082 & Up & TNF receptor associated factor 6 \\
\hline FYN & 0.536182 & $1.96 \mathrm{E}-08$ & $1.96 \mathrm{E}-08$ & 7.357891 & Up & $\begin{array}{l}\text { FYN proto-oncogene, Src family tyrosine } \\
\text { kinase }\end{array}$ \\
\hline HRAS & 0.668773 & $2.14 \mathrm{E}-08$ & $2.14 \mathrm{E}-08$ & 7.325507 & Up & HRas proto-oncogene, GTPase \\
\hline ITGB2 & 0.805182 & $2.8 \mathrm{E}-06$ & $2.8 \mathrm{E}-06$ & 5.644273 & Up & Integrin subunit beta 2 \\
\hline ZBTB16 & 1.099955 & $3.88 \mathrm{E}-06$ & $3.88 \mathrm{E}-06$ & 5.53388 & Up & Zinc finger and BTB domain containing 16 \\
\hline ABL1 & 0.468091 & $4.46 \mathrm{E}-06$ & $4.46 \mathrm{E}-06$ & 5.486138 & Up & $\begin{array}{l}\text { ABL proto-oncogene } 1 \text {, non-receptor tyros- } \\
\text { ine kinase }\end{array}$ \\
\hline CX3CR1 & 0.834227 & $6.3 \mathrm{E}-06$ & $6.3 \mathrm{E}-06$ & 5.369313 & Up & $\mathrm{C}-\mathrm{X} 3-\mathrm{C}$ motif chemokine receptor 1 \\
\hline PDCD1 & 0.880955 & $7.79 \mathrm{E}-06$ & $7.79 \mathrm{E}-06$ & 5.297093 & Up & Programmed cell death 1 \\
\hline IRF5 & 0.884364 & $1.56 \mathrm{E}-05$ & $1.56 \mathrm{E}-05$ & 5.062275 & Up & Interferon regulatory factor 5 \\
\hline IL2RG & 0.590818 & $1.7 \mathrm{E}-05$ & $1.7 \mathrm{E}-05$ & 5.032467 & Up & Interleukin 2 receptor subunit gamma \\
\hline IKZF3 & 0.467273 & $1.78 \mathrm{E}-0 \mathrm{E}-05$ & $1.78 \mathrm{E}-05$ & 5.016592 & Up & IKAROS family zinc finger 3 \\
\hline CCR1 & 1.613727 & $2.3 \mathrm{E}-05$ & $2.3 \mathrm{E}-05$ & 4.929543 & Up & $\mathrm{C}-\mathrm{C}$ motif chemokine receptor 1 \\
\hline CD99 & 0.398773 & $4.76 \mathrm{E}-05$ & $4.76 \mathrm{E}-05$ & 4.681138 & Up & CD99 molecule (Xg blood group) \\
\hline SMAD5 & 0.349818 & $5.02 \mathrm{E}-05$ & $5.02 \mathrm{E}-05$ & 4.662446 & Up & SMAD family member 5 \\
\hline CD247 & 0.51 & $5.34 \mathrm{E}-05$ & $5.34 \mathrm{E}-05$ & 4.641555 & Up & CD247 molecule \\
\hline TP53 & 0.372273 & $5.85 \mathrm{E}-05$ & $5.85 \mathrm{E}-05$ & 4.609909 & Up & Tumor protein $\mathrm{p} 53$ \\
\hline LAG3 & 0.988591 & $6.64 \mathrm{E}-05$ & $6.64 \mathrm{E}-05$ & 4.566916 & Up & Lymphocyte activating 3 \\
\hline LCP2 & 0.432682 & $6.74 \mathrm{E}-05$ & $6.74 \mathrm{E}-05$ & 4.561553 & Up & Lymphocyte cytosolic protein 2 \\
\hline SLAMF7 & 0.577409 & $8.58 \mathrm{E}-05$ & $8.58 \mathrm{E}-05$ & 4.478485 & Up & SLAM family member 7 \\
\hline TMEM173 & 0.377318 & 0.000103 & 0.000103 & 4.416144 & Up & Transmembrane protein 173 \\
\hline CUL9 & 0.4155 & 0.000119 & 0.000119 & 4.366647 & Up & Cullin 9 \\
\hline $\mathrm{C} 2$ & 1.280182 & 0.000153 & 0.000153 & 4.279415 & Up & Complement $\mathrm{C} 2$ \\
\hline GNLY & 1.067364 & 0.000167 & 0.000167 & 4.248083 & Up & Granulysin \\
\hline ATG10 & 0.490955 & 0.000234 & 0.000234 & 4.130655 & Up & Autophagy related 10 \\
\hline IKZF1 & 0.321682 & 0.000285 & 0.000285 & 4.061503 & Up & IKAROS family zinc finger 1 \\
\hline KIR_Activating_Subgroup_2 & 1.279318 & 0.00043 & 0.00043 & 3.915229 & Up & $\begin{array}{l}\text { Killer-cell immunoglobulin-like receptor- } \\
\text { Subgroup } 2\end{array}$ \\
\hline ITGAL & 0.370273 & 0.000461 & 0.000461 & 3.890576 & Up & Integrin subunit alpha L \\
\hline SERPING1 & 1.992773 & 0.00057 & 0.00057 & 3.815201 & Up & Serpin family G member 1 \\
\hline STAT1 & 1.066864 & 0.000762 & 0.000762 & 3.711251 & Up & $\begin{array}{l}\text { signal transducer and activator of transcrip- } \\
\text { tion } 1\end{array}$ \\
\hline CCRL2 & 0.621773 & 0.000844 & 0.000844 & 3.674266 & Up & $\mathrm{C}-\mathrm{C}$ motif chemokine receptor like 2 \\
\hline RUNX1 & 0.392409 & 0.001035 & 0.001035 & 3.600334 & Up & RUNX family transcription factor 1 \\
\hline KIR_Activating_Subgroup_1 & 1.243455 & 0.001444 & 0.001444 & 3.478418 & Up & $\begin{array}{l}\text { Killer-cell immunoglobulin-like receptor } \\
\text { Subgroup } 1\end{array}$ \\
\hline IFIH1 & 1.189 & 0.001492 & 0.001492 & 3.466339 & Up & Interferon induced with helicase $\mathrm{C}$ domain 1 \\
\hline GP1BB & 1.252364 & 0.001517 & 0.001517 & 3.460324 & Up & Glycoprotein Ib platelet subunit beta \\
\hline TBX21 & 0.557591 & 0.001716 & 0.001716 & 3.414736 & Up & T-box transcription factor 21 \\
\hline BST2 & 0.825727 & 0.00183 & 0.00183 & 3.390875 & Up & Bone marrow stromal cell antigen 2 \\
\hline JAK2 & 0.436727 & 0.001927 & 0.001927 & 3.371677 & Up & Janus kinase 2 \\
\hline PSMB9 & 0.490864 & 0.001928 & 0.001928 & 3.371431 & Up & Proteasome 20S subunit beta 9 \\
\hline XBP1 & 0.485455 & 0.002051 & 0.002051 & 3.348399 & Up & $\mathrm{X}$-box binding protein 1 \\
\hline GBP1 & 0.999545 & 0.003627 & 0.003627 & 3.133218 & Up & Guanylate binding protein 1 \\
\hline
\end{tabular}


Table 1 (continued)

\begin{tabular}{|c|c|c|c|c|c|c|}
\hline Gene Symbol & $\log \mathrm{FC}$ & pValue & adj.P.Val & t value & Regulation & Gene Name \\
\hline STAT4 & 0.295591 & 0.003667 & 0.003667 & 3.129061 & Up & $\begin{array}{l}\text { Signal transducer and activator of transcrip- } \\
\text { tion } 4\end{array}$ \\
\hline MAP4K1 & 0.2385 & 0.003959 & 0.003959 & 3.099644 & Up & $\begin{array}{l}\text { Mitogen-activated protein kinase kinaseki- } \\
\text { nasekinase } 1\end{array}$ \\
\hline CCND3 & 0.326591 & 0.004076 & 0.004076 & 3.08846 & Up & cyclin D3 \\
\hline LILRA6 & 0.817091 & 0.004839 & 0.004839 & 3.022071 & Up & Leukocyte immunoglobulin like receptor A6 \\
\hline GFI1 & 0.495591 & 0.005741 & 0.005741 & 2.955427 & Up & $\begin{array}{l}\text { Growth factor independent } 1 \text { transcriptional } \\
\text { repressor }\end{array}$ \\
\hline HLA-A & 0.385909 & 0.005824 & 0.005824 & 2.949825 & Up & Major histocompatibility complex, class I, A \\
\hline IL18RAP & 0.982227 & 0.006135 & 0.006135 & 2.929331 & Up & Interleukin 18 receptor accessory protein \\
\hline C1QBP & 0.264182 & 0.006145 & 0.006145 & 2.928686 & Up & Complement $\mathrm{C} 1 \mathrm{q}$ binding protein \\
\hline CCL5 & 0.3105 & 0.006563 & 0.006563 & 2.902767 & Up & $\mathrm{C}-\mathrm{C}$ motif chemokine ligand 5 \\
\hline SOCS1 & 0.704318 & 0.007115 & 0.007115 & 2.870801 & Up & Suppressor of cytokine signaling 1 \\
\hline STAT3 & 0.370545 & 0.010179 & 0.010179 & 2.72698 & Up & $\begin{array}{l}\text { Signal transducer and activator of transcrip- } \\
\text { tion } 3\end{array}$ \\
\hline CEACAM1 & 0.784909 & 0.011682 & 0.011682 & 2.670737 & Up & CEA cell adhesion molecule 1 \\
\hline TLR2 & 0.589773 & 0.013287 & 0.013287 & 2.617647 & Up & toll like receptor 2 \\
\hline KLRK1 & 0.358136 & 0.014239 & 0.014239 & 2.588898 & Up & killer cell lectin like receptor $\mathrm{K} 1$ \\
\hline MAP4K2 & 0.221227 & 0.018828 & 0.018828 & 2.471244 & Up & $\begin{array}{l}\text { Mitogen-activated protein kinase kinaseki- } \\
\text { nasekinase } 2\end{array}$ \\
\hline KLRC1 & 0.7945 & 0.019896 & 0.019896 & 2.4477 & Up & Killer cell lectin like receptor $\mathrm{C} 1$ \\
\hline ATG5 & 0.327 & 0.020137 & 0.020137 & 2.44253 & Up & Autophagy related 5 \\
\hline IL18R1 & 0.713136 & 0.020905 & 0.020905 & 2.426467 & Up & Interleukin 18 receptor 1 \\
\hline IKBKB & 0.1695 & 0.027641 & 0.027641 & 2.304841 & Up & $\begin{array}{l}\text { Inhibitor of nuclear factor kappa B kinase } \\
\text { subunit beta }\end{array}$ \\
\hline STAT5B & 0.2575 & 0.032278 & 0.032278 & 2.235871 & Up & $\begin{array}{l}\text { Signal transducer and activator of transcrip- } \\
\text { tion 5B }\end{array}$ \\
\hline MX1 & 1.111727 & 0.032817 & 0.032817 & 2.228441 & Up & MX dynamin like GTPase 1 \\
\hline IRF7 & 0.898909 & 0.033248 & 0.033248 & 2.222589 & Up & Interferon regulatory factor 7 \\
\hline TRAF2 & 0.171955 & 0.034273 & 0.034273 & 2.208908 & Up & TNF receptor associated factor 2 \\
\hline IFI35 & 0.725909 & 0.035093 & 0.035093 & 2.198229 & Up & Interferon induced protein 35 \\
\hline IKBKE & 0.236545 & 0.035149 & 0.035149 & 2.197514 & Up & $\begin{array}{l}\text { Inhibitor of nuclear factor kappa B kinase } \\
\text { subunit epsilon }\end{array}$ \\
\hline CLEC7A & 0.421227 & 0.037828 & 0.037828 & 2.164155 & Up & C-type lectin domain containing 7A \\
\hline LTB4R & 0.465227 & 0.03902 & 0.03902 & 2.149988 & Up & Leukotriene B4 receptor \\
\hline GZMB & 0.437364 & 0.040654 & 0.040654 & 2.131179 & Up & Granzyme B \\
\hline NLRP3 & 0.290364 & 0.04286 & 0.04286 & 2.106829 & Up & NLR family pyrin domain containing 3 \\
\hline LILRB2 & 0.299364 & 0.042931 & 0.042931 & 2.106059 & Up & Leukocyte immunoglobulin like receptor B2 \\
\hline IFNAR2 & 0.184136 & 0.048633 & 0.048633 & 2.047965 & Up & Interferon alpha and beta receptor subunit 2 \\
\hline KLRD1 & 0.4455 & 0.049759 & 0.049759 & 2.037205 & Up & Killer cell lectin like receptor D1 \\
\hline CD19 & -1.7175 & $5.18 \mathrm{E}-16$ & $5.18 \mathrm{E}-16$ & -14.7177 & Down & CD19 molecule \\
\hline CD22 & -1.757 & $4.93 \mathrm{E}-15$ & $4.93 \mathrm{E}-15$ & -13.592 & Down & CD22 molecule \\
\hline MS4A1 & -1.67741 & $1.67 \mathrm{E}-14$ & $1.67 \mathrm{E}-14$ & -13.0082 & Down & Membrane spanning 4-domains A1 \\
\hline CD45RB & -0.94709 & $4.17 \mathrm{E}-14$ & $4.17 \mathrm{E}-14$ & -12.5832 & Down & $\begin{array}{l}\text { Receptor-Type Tyrosine-Protein Phosphatase } \\
\text { C }\end{array}$ \\
\hline IL6R & -2.0375 & $1.05 \mathrm{E}-13$ & $1.05 \mathrm{E}-13$ & -12.164 & Down & interleukin 6 receptor \\
\hline TNFRSF13C & -1.46186 & $2.18 \mathrm{E}-13$ & $2.18 \mathrm{E}-13$ & -11.8375 & Down & TNF receptor superfamily member $13 \mathrm{C}$ \\
\hline PAX5 & -1.37636 & $2.92 \mathrm{E}-13$ & $2.92 \mathrm{E}-13$ & -11.7085 & Down & Paired box 5 \\
\hline CD79A & -1.29577 & $2.53 \mathrm{E}-12$ & $2.53 \mathrm{E}-12$ & -10.7841 & Down & CD79a molecule \\
\hline ARHGDIB & -0.65064 & $3.68 \mathrm{E}-12$ & $3.68 \mathrm{E}-12$ & -10.6278 & Down & Rho GDP dissociation inhibitor beta \\
\hline HLA-DQB1 & -4.88305 & $5.18 \mathrm{E}-12$ & $5.18 \mathrm{E}-12$ & -10.4865 & Down & $\begin{array}{l}\text { Major histocompatibility complex, class II, } \\
\text { DQ beta } 1\end{array}$ \\
\hline
\end{tabular}


Table 1 (continued)

\begin{tabular}{|c|c|c|c|c|c|c|}
\hline Gene Symbol & $\log \mathrm{FC}$ & pValue & adj.P.Val & t value & Regulation & Gene Name \\
\hline HLA-DQA1 & -6.1155 & $1.8 \mathrm{E}-11$ & $1.8 \mathrm{E}-11$ & -9.98021 & Down & $\begin{array}{l}\text { Major histocompatibility complex, class II, } \\
\text { DQ alpha } 1\end{array}$ \\
\hline MAPKAPK2 & -0.99755 & $5.49 \mathrm{E}-11$ & $5.49 \mathrm{E}-11$ & -9.53915 & Down & MAPK activated protein kinase 2 \\
\hline SLAMF6 & -0.87414 & $2.16 \mathrm{E}-10$ & $2.16 \mathrm{E}-10$ & -9.01008 & Down & SLAM family member 6 \\
\hline PTGER4 & -0.76932 & $2.43 \mathrm{E}-10$ & $2.43 \mathrm{E}-10$ & -8.96519 & Down & Prostaglandin E receptor 4 \\
\hline CD79B & -0.84082 & $2.75 \mathrm{E}-10$ & $2.75 \mathrm{E}-10$ & -8.91788 & Down & CD79b molecule \\
\hline CD97 & -1.1985 & $3.22 \mathrm{E}-10$ & $3.22 \mathrm{E}-10$ & -8.85915 & Down & Leukocyte antigen CD97 \\
\hline IL1RL2 & -1.04873 & $3.57 \mathrm{E}-10$ & $3.57 \mathrm{E}-10$ & -8.81964 & Down & Interleukin 1 receptor like 2 \\
\hline CD1A & -1.07114 & $3.6 \mathrm{E}-10$ & $3.6 \mathrm{E}-10$ & -8.81626 & Down & CD1a molecule \\
\hline IL1RAP & -1.43418 & $8.92 \mathrm{E}-10$ & $8.92 \mathrm{E}-10$ & -8.47672 & Down & Interleukin 1 receptor accessory protein \\
\hline TNFSF12 & -0.53718 & $2.42 \mathrm{E}-09$ & $2.42 \mathrm{E}-09$ & -8.10915 & Down & TNF superfamily member 12 \\
\hline AICDA & -0.94759 & $2.89 \mathrm{E}-09$ & $2.89 \mathrm{E}-09$ & -8.04473 & Down & Activation induced cytidinedeaminase \\
\hline MBP & -0.77614 & $4.02 \mathrm{E}-09$ & $4.02 \mathrm{E}-09$ & -7.92468 & Down & myelin basic protein \\
\hline TRAF4 & -0.67777 & $5.69 \mathrm{E}-09$ & $5.69 \mathrm{E}-09$ & -7.79888 & Down & TNF receptor associated factor 4 \\
\hline MIF & -0.59309 & $5.94 \mathrm{E}-09$ & $5.94 \mathrm{E}-09$ & -7.78344 & Down & Macrophage migration inhibitory factor \\
\hline СCBP2 & -1.34027 & $6.31 \mathrm{E}-09$ & $6.31 \mathrm{E}-09$ & -7.76207 & Down & Chemokine-binding protein 2 \\
\hline CCL22 & -0.68986 & $8.3 \mathrm{E}-09$ & $8.3 \mathrm{E}-09$ & -7.66358 & Down & $\mathrm{C}-\mathrm{C}$ motif chemokine ligand 22 \\
\hline HLA-DRA & -1.87223 & $9.28 \mathrm{E}-09$ & $9.28 \mathrm{E}-09$ & -7.62328 & Down & $\begin{array}{l}\text { Major histocompatibility complex, class II, } \\
\text { DR alpha }\end{array}$ \\
\hline FCER1A & -2.15768 & $9.79 \mathrm{E}-09$ & $9.79 \mathrm{E}-09$ & -7.60411 & Down & Fc fragment of IgE receptor Ia \\
\hline LILRB5 & -0.76595 & $1.11 \mathrm{E}-08$ & $1.11 \mathrm{E}-08$ & -7.55809 & Down & Leukocyte immunoglobulin like receptor B5 \\
\hline CCL15 & -0.7105 & $1.48 \mathrm{E}-08$ & $1.48 \mathrm{E}-08$ & -7.4567 & Down & $\mathrm{C}-\mathrm{C}$ motif chemokine ligand 15 \\
\hline IL12B & -0.99886 & $1.5 \mathrm{E}-08$ & $1.5 \mathrm{E}-08$ & -7.45174 & Down & Interleukin 12B \\
\hline TFRC & -1.07782 & $1.61 \mathrm{E}-08$ & $1.61 \mathrm{E}-08$ & -7.42698 & Down & Transferrin receptor \\
\hline EBI3 & -0.72195 & $1.86 \mathrm{E}-08$ & $1.86 \mathrm{E}-08$ & -7.37653 & Down & Epstein-Barr virus induced 3 \\
\hline IL4 & -0.69941 & $2.72 \mathrm{E}-08$ & $2.72 \mathrm{E}-08$ & -7.24189 & Down & Interleukin 4 \\
\hline ICAM2 & -0.74745 & $2.86 \mathrm{E}-08$ & $2.86 \mathrm{E}-08$ & -7.22422 & Down & Intercellular adhesion molecule 2 \\
\hline KLRAP1 & -1.0865 & $3.09 \mathrm{E}-08$ & $3.09 \mathrm{E}-08$ & -7.19683 & Down & $\begin{array}{l}\text { Killer cell lectin-like receptor subfamily A } \\
\text { pseudogene } 1\end{array}$ \\
\hline CD40 & -0.79436 & $3.29 \mathrm{E}-08$ & $3.29 \mathrm{E}-08$ & -7.17439 & Down & CD40 molecule \\
\hline IL22RA2 & -0.6365 & $4.34 \mathrm{E}-08$ & $4.34 \mathrm{E}-08$ & -7.07766 & Down & Interleukin 22 receptor subunit alpha 2 \\
\hline IL2 & -0.64395 & $4.65 \mathrm{E}-08$ & $4.65 \mathrm{E}-08$ & -7.05298 & Down & Interleukin 2 \\
\hline IL29 & -0.64395 & $4.65 \mathrm{E}-08$ & $4.65 \mathrm{E}-08$ & -7.05298 & Down & Interleukin 29 \\
\hline $\mathrm{CD} 3 \mathrm{E}$ & -0.73018 & $5.24 \mathrm{E}-08$ & $5.24 \mathrm{E}-08$ & -7.01159 & Down & CD3e molecule \\
\hline CD55 & -0.92114 & $5.29 \mathrm{E}-08$ & $5.29 \mathrm{E}-08$ & -7.00828 & Down & CD55 molecule (Cromer blood group) \\
\hline IL19 & -0.6555 & $5.33 \mathrm{E}-08$ & $5.33 \mathrm{E}-08$ & -7.00515 & Down & Interleukin 19 \\
\hline NOS2 & -0.81432 & $5.6 \mathrm{E}-08$ & $5.6 \mathrm{E}-08$ & -6.98829 & Down & Nitric oxide synthase 2 \\
\hline C4BPA & -1.83359 & $5.78 \mathrm{E}-08$ & $5.78 \mathrm{E}-08$ & -6.97697 & Down & $\begin{array}{l}\text { Complement component } 4 \text { binding protein } \\
\text { alpha }\end{array}$ \\
\hline CCL26 & -0.57677 & $6.5 \mathrm{E}-08$ & $6.5 \mathrm{E}-08$ & -6.93596 & Down & $\mathrm{C}-\mathrm{C}$ motif chemokine ligand 26 \\
\hline $\mathrm{CDH} 5$ & -0.60586 & $7.09 \mathrm{E}-08$ & $7.09 \mathrm{E}-08$ & -6.90577 & Down & Cadherin 5 \\
\hline IL9 & -0.60586 & $7.09 \mathrm{E}-08$ & $7.09 \mathrm{E}-08$ & -6.90577 & Down & Interleukin 9 \\
\hline FCGRT & -0.75709 & $8.18 \mathrm{E}-08$ & $8.18 \mathrm{E}-08$ & -6.85603 & Down & $\mathrm{Fc}$ fragment of $\mathrm{IgG}$ receptor and transporter \\
\hline $\mathrm{C} 8 \mathrm{~B}$ & -0.6555 & $1.1 \mathrm{E}-07$ & $1.1 \mathrm{E}-07$ & -6.75159 & Down & Complement $\mathrm{C} 8$ beta chain \\
\hline IL5 & -0.64086 & $1.16 \mathrm{E}-07$ & $1.16 \mathrm{E}-07$ & -6.7344 & Down & Interleukin 5 \\
\hline PIGR & -0.66695 & $1.33 \mathrm{E}-07$ & $1.33 \mathrm{E}-07$ & -6.68821 & Down & Polymeric immunoglobulin receptor \\
\hline XCL1 & -1.06423 & $1.98 \mathrm{E}-07$ & $1.98 \mathrm{E}-07$ & -6.54928 & Down & $\mathrm{X}-\mathrm{C}$ motif chemokine ligand 1 \\
\hline AIRE & -0.78032 & $2.01 \mathrm{E}-07$ & $2.01 \mathrm{E}-07$ & -6.5441 & Down & Autoimmune regulator \\
\hline IL3 & -0.60723 & $2.08 \mathrm{E}-07$ & $2.08 \mathrm{E}-07$ & -6.53283 & Down & Interleukin 3 \\
\hline CCL16 & -0.6195 & $2.31 \mathrm{E}-07$ & $2.31 \mathrm{E}-07$ & -6.4973 & Down & $\mathrm{C}-\mathrm{C}$ motif chemokine ligand 16 \\
\hline
\end{tabular}


Table 1 (continued)

\begin{tabular}{|c|c|c|c|c|c|c|}
\hline Gene Symbol & $\operatorname{logFC}$ & pValue & adj.P.Val & $\mathrm{t}$ value & Regulation & Gene Name \\
\hline CCL7 & -0.6195 & $2.31 \mathrm{E}-07$ & $2.31 \mathrm{E}-07$ & -6.4973 & Down & $\mathrm{C}-\mathrm{C}$ motif chemokine ligand 7 \\
\hline CSF2 & -0.6195 & $2.31 \mathrm{E}-07$ & $2.31 \mathrm{E}-07$ & -6.4973 & Down & Colony stimulating factor 2 \\
\hline ITLN2 & -0.6195 & $2.31 \mathrm{E}-07$ & $2.31 \mathrm{E}-07$ & -6.4973 & Down & Intelectin 2 \\
\hline THY1 & -0.6195 & $2.31 \mathrm{E}-07$ & $2.31 \mathrm{E}-07$ & -6.4973 & Down & Thy- 1 cell surface antigen \\
\hline IL21 & -0.70245 & $3.28 \mathrm{E}-07$ & $3.28 \mathrm{E}-07$ & -6.37574 & Down & Interleukin 21 \\
\hline BCL2 & -0.7385 & $4.11 \mathrm{E}-07$ & $4.11 \mathrm{E}-07$ & -6.29854 & Down & BCL2 apoptosis regulator \\
\hline EDNRB & -0.60541 & $4.11 \mathrm{E}-07$ & $4.11 \mathrm{E}-07$ & -6.29833 & Down & Endothelin receptor type B \\
\hline CCR6 & -0.88205 & $4.78 \mathrm{E}-07$ & $4.78 \mathrm{E}-07$ & -6.24666 & Down & $\mathrm{C}-\mathrm{C}$ motif chemokine receptor 6 \\
\hline TIRAP & -0.70182 & $5.91 \mathrm{E}-07$ & $5.91 \mathrm{E}-07$ & -6.17398 & Down & TIR domain containing adaptor protein \\
\hline STAT6 & -0.52186 & $6.37 \mathrm{E}-07$ & $6.37 \mathrm{E}-07$ & -6.14848 & Down & $\begin{array}{l}\text { Signal transducer and activator of transcrip- } \\
\text { tion } 6\end{array}$ \\
\hline PSMB10 & -0.43945 & $6.43 \mathrm{E}-07$ & $6.43 \mathrm{E}-07$ & -6.14519 & Down & Proteasome 20S subunit beta 10 \\
\hline SKI & -0.52182 & $7.16 \mathrm{E}-07$ & $7.16 \mathrm{E}-07$ & -6.10886 & Down & SKI proto-oncogene \\
\hline RAG2 & -0.56723 & $8.47 \mathrm{E}-07$ & $8.47 \mathrm{E}-07$ & -6.05149 & Down & Recombination activating 2 \\
\hline CD209 & -0.7285 & $8.56 \mathrm{E}-07$ & $8.56 \mathrm{E}-07$ & -6.04758 & Down & CD209 molecule \\
\hline VTN & -0.68305 & $9.43 \mathrm{E}-07$ & $9.43 \mathrm{E}-07$ & -6.01488 & Down & Vitronectin \\
\hline IFNB1 & -0.57768 & $9.97 \mathrm{E}-07$ & $9.97 \mathrm{E}-07$ & -5.99573 & Down & Interferon beta 1 \\
\hline EOMES & -0.77505 & $1.47 \mathrm{E}-06$ & $1.47 \mathrm{E}-06$ & -5.8643 & Down & Eomesodermin \\
\hline CD74 & -0.69905 & $1.52 \mathrm{E}-06$ & $1.52 \mathrm{E}-06$ & -5.85145 & Down & CD74 molecule \\
\hline CCR10 & -0.60368 & $1.7 \mathrm{E}-06$ & $1.7 \mathrm{E}-06$ & -5.81503 & Down & $\mathrm{C}-\mathrm{C}$ motif chemokine receptor 10 \\
\hline LGALS3 & -1.12309 & $1.85 \mathrm{E}-06$ & $1.85 \mathrm{E}-06$ & -5.78563 & Down & Galectin 3 \\
\hline PDGFB & -0.64241 & $1.86 \mathrm{E}-06$ & $1.86 \mathrm{E}-06$ & -5.78395 & Down & Platelet derived growth factor subunit B \\
\hline ICAM4 & -0.96814 & $1.96 \mathrm{E}-06$ & $1.96 \mathrm{E}-06$ & -5.76557 & Down & $\begin{array}{l}\text { Intercellular adhesion molecule } 4 \text { (Land- } \\
\text { steiner-Wiener blood group) }\end{array}$ \\
\hline ICOSLG & -0.74791 & $2.07 \mathrm{E}-06$ & $2.07 \mathrm{E}-06$ & -5.74698 & Down & Inducible $\mathrm{T}$ cell costimulator ligand \\
\hline C9 & -0.52132 & $2.66 \mathrm{E}-06$ & $2.66 \mathrm{E}-06$ & -5.66169 & Down & Complement C9 \\
\hline IL16 & -0.45973 & $2.88 \mathrm{E}-06$ & $2.88 \mathrm{E}-06$ & -5.63467 & Down & Interleukin 16 \\
\hline RELA & -0.47205 & $3.09 \mathrm{E}-06$ & $3.09 \mathrm{E}-06$ & -5.61097 & Down & RELA proto-oncogene, NF-kB subunit \\
\hline DEFB1 & -0.8105 & $3.38 \mathrm{E}-06$ & $3.38 \mathrm{E}-06$ & -5.58051 & Down & Defensin beta 1 \\
\hline IL13RA1 & -0.90159 & $3.41 \mathrm{E}-06$ & $3.41 \mathrm{E}-06$ & -5.57755 & Down & Interleukin 13 receptor subunit alpha 1 \\
\hline HLA-DOB & -1.24595 & $3.65 \mathrm{E}-06$ & $3.65 \mathrm{E}-06$ & -5.55493 & Down & $\begin{array}{l}\text { Major histocompatibility complex, class II, } \\
\text { DO beta }\end{array}$ \\
\hline KIR3DL3 & -0.56905 & $3.78 \mathrm{E}-06$ & $3.78 \mathrm{E}-06$ & -5.54244 & Down & $\begin{array}{l}\text { Killer cell immunoglobulin like receptor, } \\
\text { three Ig domains and long cytoplasmic } \\
\text { tail } 3\end{array}$ \\
\hline HLA-C & -0.93941 & $3.97 \mathrm{E}-06$ & $3.97 \mathrm{E}-06$ & -5.52568 & Down & Major histocompatibility complex, class I, C \\
\hline KLRB1 & -1.79632 & $5.31 \mathrm{E}-06$ & $5.31 \mathrm{E}-06$ & -5.42732 & Down & Killer cell lectin like receptor B1 \\
\hline HLA-DMB & -0.85005 & $5.86 \mathrm{E}-06$ & $5.86 \mathrm{E}-06$ & -5.39367 & Down & $\begin{array}{l}\text { Major histocompatibility complex, class II, } \\
\text { DM beta }\end{array}$ \\
\hline KLRF2 & -0.53859 & $6 \mathrm{E}-06$ & $6 \mathrm{E}-06$ & -5.3857 & Down & Killer cell lectin like receptor F2 \\
\hline ICAM3 & -0.66718 & $6.38 \mathrm{E}-06$ & $6.38 \mathrm{E}-06$ & -5.36481 & Down & Intercellular adhesion molecule 3 \\
\hline IL28A & -0.55718 & $7.03 \mathrm{E}-06$ & $7.03 \mathrm{E}-06$ & -5.33194 & Down & Interleukin $28 \mathrm{~A}$ \\
\hline SIGIRR & -0.41545 & $7.52 \mathrm{E}-06$ & $7.52 \mathrm{E}-06$ & -5.30925 & Down & Single Ig and TIR domain containing \\
\hline MASP2 & -0.68927 & $7.93 \mathrm{E}-06$ & $7.93 \mathrm{E}-06$ & -5.29109 & Down & Mannan binding lectin serine peptidase 2 \\
\hline CSF2RB & -1.12332 & $8.36 \mathrm{E}-06$ & $8.36 \mathrm{E}-06$ & -5.27318 & Down & $\begin{array}{l}\text { Colony stimulating factor } 2 \text { receptor beta } \\
\text { common subunit }\end{array}$ \\
\hline CD46 & -0.59905 & $9.11 \mathrm{E}-06$ & $9.11 \mathrm{E}-06$ & -5.24408 & Down & CD46 molecule \\
\hline S1PR1 & -0.60436 & $9.23 \mathrm{E}-06$ & $9.23 \mathrm{E}-06$ & -5.23966 & Down & Sphingosine-1-phosphate receptor 1 \\
\hline TLR9 & -0.56841 & $1.05 \mathrm{E}-05$ & $1.05 \mathrm{E}-05$ & -5.19438 & Down & Toll like receptor 9 \\
\hline HLA-DPB 1 & -0.70064 & $1.07 \mathrm{E}-05$ & $1.07 \mathrm{E}-05$ & -5.19046 & Down & $\begin{array}{l}\text { Major histocompatibility complex, class II, } \\
\text { DP beta } 1\end{array}$ \\
\hline
\end{tabular}


Table 1 (continued)

\begin{tabular}{|c|c|c|c|c|c|c|}
\hline Gene Symbol & $\log \mathrm{FC}$ & pValue & adj.P.Val & $\mathrm{t}$ value & Regulation & Gene Name \\
\hline$\overline{\text { CCL13 }}$ & -0.57018 & $1.16 \mathrm{E}-05$ & $1.16 \mathrm{E}-05$ & -5.16161 & Down & $\mathrm{C}-\mathrm{C}$ motif chemokine ligand 13 \\
\hline PLA2G2E & -0.67155 & $1.19 \mathrm{E}-05$ & $1.19 \mathrm{E}-05$ & -5.15362 & Down & Phospholipase A2 group IIE \\
\hline IL20 & -0.67032 & $1.29 \mathrm{E}-05$ & $1.29 \mathrm{E}-05$ & -5.12687 & Down & Interleukin 20 \\
\hline PTAFR & -0.91373 & $1.37 \mathrm{E}-05$ & $1.37 \mathrm{E}-05$ & -5.10671 & Down & Platelet activating factor receptor \\
\hline TGFBI & -0.59209 & $1.44 \mathrm{E}-05$ & $1.44 \mathrm{E}-05$ & -5.08943 & Down & Transforming growth factor beta induced \\
\hline IL26 & -0.50514 & $1.52 \mathrm{E}-05$ & $1.52 \mathrm{E}-05$ & -5.07029 & Down & Interleukin 26 \\
\hline IFNAR1 & -0.78755 & $2.03 \mathrm{E}-05$ & $2.03 \mathrm{E}-05$ & -4.97214 & Down & Interferon alpha and beta receptor subunit 1 \\
\hline LTA & -0.56282 & $2.17 \mathrm{E}-05$ & $2.17 \mathrm{E}-05$ & -4.94915 & Down & Lymphotoxin alpha \\
\hline FCGR2B & -0.85355 & $2.4 \mathrm{E}-05$ & $2.4 \mathrm{E}-05$ & -4.91443 & Down & Fc fragment of IgG receptor IIb \\
\hline EGR1 & -0.63082 & $2.41 \mathrm{E}-05$ & $2.41 \mathrm{E}-05$ & -4.9133 & Down & Early growth response 1 \\
\hline CD86 & -0.76691 & $2.54 \mathrm{E}-05$ & $2.54 \mathrm{E}-05$ & -4.89525 & Down & CD86 molecule \\
\hline $\mathrm{CD} 82$ & -0.99568 & $2.76 \mathrm{E}-05$ & $2.76 \mathrm{E}-05$ & -4.86674 & Down & CD82 molecule \\
\hline CD34 & -0.56045 & $2.79 \mathrm{E}-05$ & $2.79 \mathrm{E}-05$ & -4.86293 & Down & CD34 molecule \\
\hline TRAF3 & -0.46214 & $2.87 \mathrm{E}-05$ & $2.87 \mathrm{E}-05$ & -4.85429 & Down & TNF receptor associated factor 3 \\
\hline CTLA4 & -0.53577 & $3 \mathrm{E}-05$ & $3 E-05$ & -4.83886 & Down & $\begin{array}{l}\text { Cytotoxic T-Lymphocyte Associated Protein } \\
4\end{array}$ \\
\hline HAMP & -0.61205 & $3 E-05$ & $3 E-05$ & -4.83856 & Down & Hepcidin antimicrobial peptide \\
\hline EGR2 & -0.78873 & $3.15 \mathrm{E}-05$ & $3.15 \mathrm{E}-05$ & -4.82228 & Down & Early growth response 2 \\
\hline ICAM5 & -0.53214 & $3.22 \mathrm{E}-05$ & $3.22 \mathrm{E}-05$ & -4.81493 & Down & Intercellular adhesion molecule 5 \\
\hline CSF1R & -0.70273 & $3.45 \mathrm{E}-05$ & $3.45 \mathrm{E}-05$ & -4.79056 & Down & Colony stimulating factor 1 receptor \\
\hline NT5E & -0.85836 & $3.68 \mathrm{E}-05$ & $3.68 \mathrm{E}-05$ & -4.76884 & Down & 5'-nucleotidase ecto \\
\hline IL7 & -0.74464 & $4.3 \mathrm{E}-05$ & $4.3 \mathrm{E}-05$ & -4.71548 & Down & Interleukin 7 \\
\hline CTSS & -0.62991 & $4.34 \mathrm{E}-05$ & $4.34 \mathrm{E}-05$ & -4.71277 & Down & Cathepsin S \\
\hline IL17B & -0.55268 & $4.82 \mathrm{E}-05$ & $4.82 \mathrm{E}-05$ & -4.6769 & Down & Interleukin 17B \\
\hline CR2 & -1.00786 & $4.86 \mathrm{E}-05$ & $4.86 \mathrm{E}-05$ & -4.67369 & Down & Complement $\mathrm{C} 3 \mathrm{~d}$ receptor 2 \\
\hline CD44 & -0.35164 & $5.25 \mathrm{E}-05$ & $5.25 \mathrm{E}-05$ & -4.64701 & Down & CD44 molecule (Indian blood group) \\
\hline PLA2G2A & -0.53655 & $5.77 \mathrm{E}-05$ & $5.77 \mathrm{E}-05$ & -4.61492 & Down & Phospholipase A2 group IIA \\
\hline BTK & -0.45495 & $5.87 \mathrm{E}-05$ & $5.87 \mathrm{E}-05$ & -4.60896 & Down & Bruton tyrosine kinase \\
\hline C6 & -0.65964 & $5.95 \mathrm{E}-05$ & $5.95 \mathrm{E}-05$ & -4.60439 & Down & Complement C6 \\
\hline IRGM & -0.62291 & $6.21 \mathrm{E}-05$ & $6.21 \mathrm{E}-05$ & -4.58953 & Down & Immunity related GTPase $M$ \\
\hline IL22 & -0.65314 & $7.15 \mathrm{E}-05$ & $7.15 \mathrm{E}-05$ & -4.54121 & Down & Interleukin 22 \\
\hline CEBPB & -0.88709 & $7.69 \mathrm{E}-05$ & $7.69 \mathrm{E}-05$ & -4.51625 & Down & CCAAT enhancer binding protein beta \\
\hline IL6 & -0.69105 & $7.91 \mathrm{E}-05$ & $7.91 \mathrm{E}-05$ & -4.50639 & Down & Interleukin 6 \\
\hline IFNA2 & -0.77586 & $8.23 \mathrm{E}-05$ & $8.23 \mathrm{E}-05$ & -4.49296 & Down & Interferon alpha 2 \\
\hline TGFBR1 & -0.40336 & $8.66 \mathrm{E}-05$ & $8.66 \mathrm{E}-05$ & -4.47511 & Down & Transforming growth factor beta receptor 1 \\
\hline IL17F & -0.54009 & $8.83 \mathrm{E}-05$ & $8.83 \mathrm{E}-05$ & -4.4686 & Down & Interleukin $17 \mathrm{~F}$ \\
\hline IL28A/B & -0.55282 & 0.000163 & 0.000163 & -4.25674 & Down & Interleukin 28A/B \\
\hline IL17A & -0.53723 & 0.00018 & 0.00018 & -4.22236 & Down & Interleukin 17A \\
\hline LILRA2 & -0.68495 & 0.000195 & 0.000195 & -4.194 & Down & Leukocyte immunoglobulin like receptor A2 \\
\hline LILRA3 & -2.33691 & 0.000203 & 0.000203 & -4.18055 & Down & Leukocyte immunoglobulin like receptor A3 \\
\hline VCAM1 & -0.60605 & 0.000219 & 0.000219 & -4.15259 & Down & Vascular cell adhesion molecule 1 \\
\hline SPP1 & -0.6555 & 0.000221 & 0.000221 & -4.1496 & Down & Secreted phosphoprotein 1 \\
\hline CARD9 & -0.55227 & 0.000269 & 0.000269 & -4.0818 & Down & $\begin{array}{l}\text { Caspase recruitment domain family member } \\
9\end{array}$ \\
\hline LCK & -0.40364 & 0.000299 & 0.000299 & -4.04398 & Down & $\begin{array}{l}\text { LCK proto-oncogene, Src family tyrosine } \\
\text { kinase }\end{array}$ \\
\hline SELE & -0.52332 & 0.000306 & 0.000306 & -4.03551 & Down & selectin $\mathrm{E}$ \\
\hline SLC2A1 & -0.44118 & 0.00036 & 0.00036 & -3.97865 & Down & solute carrier family 2 member 1 \\
\hline LIF & -0.50555 & 0.000368 & 0.000368 & -3.97037 & Down & LIF interleukin 6 family cytokine \\
\hline IDO1 & -0.95541 & 0.000417 & 0.000417 & -3.92685 & Down & Indoleamine 2,3-dioxygenase 1 \\
\hline
\end{tabular}


Table 1 (continued)

\begin{tabular}{|c|c|c|c|c|c|c|}
\hline Gene Symbol & $\log \mathrm{FC}$ & pValue & adj.P.Val & $\mathrm{t}$ value & Regulation & Gene Name \\
\hline TNFRSF10C & -0.88564 & 0.000471 & 0.000471 & -3.88294 & Down & TNF receptor superfamily member $10 \mathrm{c}$ \\
\hline IL23A & -0.87541 & 0.000557 & 0.000557 & -3.82374 & Down & interleukin 23 subunit alpha \\
\hline CD80 & -0.58964 & 0.000567 & 0.000567 & -3.81702 & Down & CD80 molecule \\
\hline GPR183 & -1.088 & 0.00057 & 0.00057 & -3.81527 & Down & G protein-coupled receptor 183 \\
\hline $\mathrm{C} 1 \mathrm{R}$ & -0.41727 & 0.000592 & 0.000592 & -3.8018 & Down & Complement $\mathrm{C} 1 \mathrm{r}$ \\
\hline CCL11 & -0.55055 & 0.000611 & 0.000611 & -3.79064 & Down & $\mathrm{C}-\mathrm{C}$ motif chemokine ligand 11 \\
\hline HLA-DPA1 & -0.6385 & 0.000637 & 0.000637 & -3.77577 & Down & $\begin{array}{l}\text { major histocompatibility complex, class II, } \\
\text { DP alpha } 1\end{array}$ \\
\hline IFNG & -0.877 & 0.00073 & 0.00073 & -3.72658 & Down & Interferon gamma \\
\hline DUSP4 & -0.70741 & 0.000789 & 0.000789 & -3.69861 & Down & Dual specificity phosphatase 4 \\
\hline IL27 & -0.44905 & 0.000852 & 0.000852 & -3.67089 & Down & Interleukin 27 \\
\hline CD48 & -1.15805 & 0.000953 & 0.000953 & -3.6304 & Down & CD48 molecule \\
\hline FN1 & -0.56177 & 0.001005 & 0.001005 & -3.61111 & Down & fibronectin 1 \\
\hline CD244 & -0.39427 & 0.001018 & 0.001018 & -3.60646 & Down & CD244 molecule \\
\hline РPBP & -1.35464 & 0.001091 & 0.001091 & -3.58105 & Down & pro-platelet basic protein \\
\hline IL13 & -0.599 & 0.001204 & 0.001204 & -3.54515 & Down & interleukin 13 \\
\hline CCL24 & -0.45395 & 0.001221 & 0.001221 & -3.53987 & Down & $\mathrm{C}-\mathrm{C}$ motif chemokine ligand 24 \\
\hline GATA3 & -0.55041 & 0.001228 & 0.001228 & -3.53797 & Down & GATA binding protein 3 \\
\hline BID & -0.46636 & 0.001281 & 0.001281 & -3.52243 & Down & $\mathrm{BH} 3$ interacting domain death agonist \\
\hline BCL2L11 & -0.40091 & 0.001565 & 0.001565 & -3.44881 & Down & BCL2 like 11 \\
\hline KIT & -0.52868 & 0.001589 & 0.001589 & -3.44316 & Down & $\begin{array}{l}\text { KIT proto-oncogene, receptor tyrosine } \\
\text { kinase }\end{array}$ \\
\hline MME & -1.00591 & 0.001596 & 0.001596 & -3.44151 & Down & Membrane metalloendopeptidase \\
\hline ZEB1 & -0.38082 & 0.001667 & 0.001667 & -3.42548 & Down & Zinc finger E-box binding homeobox 1 \\
\hline $\mathrm{C} 4 \mathrm{~A} / \mathrm{B}$ & -0.62314 & 0.001738 & 0.001738 & -3.40994 & Down & Complement C4A/B \\
\hline FCAR & -0.50423 & 0.00198 & 0.00198 & -3.36169 & Down & $\mathrm{Fc}$ fragment of $\mathrm{IgA}$ receptor \\
\hline BTLA & -0.45623 & 0.002033 & 0.002033 & -3.35183 & Down & B and T lymphocyte associated \\
\hline TAGAP & -0.38886 & 0.002261 & 0.002261 & -3.31206 & Down & $\begin{array}{l}\text { T cell activation RhoGTPase activating } \\
\text { protein }\end{array}$ \\
\hline CD83 & -0.39914 & 0.002292 & 0.002292 & -3.307 & Down & CD83 molecule \\
\hline SELPLG & -0.37564 & 0.002362 & 0.002362 & -3.29575 & Down & Selectin P ligand \\
\hline B3GAT1 & -0.66791 & 0.002423 & 0.002423 & -3.28614 & Down & Beta-1,3-glucuronyltransferase 1 \\
\hline CCRL1 & -0.39195 & 0.002543 & 0.002543 & -3.26788 & Down & $\mathrm{C}-\mathrm{C}$ chemokine receptor type 11 \\
\hline TNFRSF14 & -0.29986 & 0.002557 & 0.002557 & -3.26588 & Down & TNF receptor superfamily member 14 \\
\hline CSF3R & -0.65318 & 0.003003 & 0.003003 & -3.20507 & Down & Colony stimulating factor 3 receptor \\
\hline CD9 & -0.87091 & 0.003004 & 0.003004 & -3.20497 & Down & CD9 molecule \\
\hline $\mathrm{C} 1 \mathrm{~S}$ & -0.52059 & 0.003211 & 0.003211 & -3.17969 & Down & complement $\mathrm{C} 1 \mathrm{~s}$ \\
\hline PECAM1 & -0.30873 & 0.003251 & 0.003251 & -3.17496 & Down & $\begin{array}{l}\text { platelet and endothelial cell adhesion mol- } \\
\text { ecule } 1\end{array}$ \\
\hline DEFB103B & -0.4315 & 0.003466 & 0.003466 & -3.15055 & Down & Defensin beta 103B \\
\hline ITLN1 & -1.17682 & 0.003488 & 0.003488 & -3.14815 & Down & Intelectin 1 \\
\hline CXCL13 & -0.46586 & 0.003554 & 0.003554 & -3.14101 & Down & $\mathrm{C}-\mathrm{X}-\mathrm{C}$ motif chemokine ligand 13 \\
\hline RAG1 & -0.47836 & 0.003917 & 0.003917 & -3.10376 & Down & Recombination activating 1 \\
\hline TNFRSF4 & -0.55123 & 0.004154 & 0.004154 & -3.08112 & Down & TNF receptor superfamily member 4 \\
\hline C14orf166 & -0.35727 & 0.004255 & 0.004255 & -3.07189 & Down & Chromosome 14 open reading frame 166 \\
\hline IL10RA & -0.26059 & 0.004423 & 0.004423 & -3.0569 & Down & Interleukin 10 receptor subunit alpha \\
\hline POU2F2 & -0.292 & 0.004614 & 0.004614 & -3.04059 & Down & POU class 2 homeobox 2 \\
\hline $\mathrm{C} 7$ & -0.41223 & 0.004688 & 0.004688 & -3.03441 & Down & complement C7 \\
\hline RORC & -0.52768 & 0.005006 & 0.005006 & -3.00891 & Down & RAR related orphan receptor $\mathrm{C}$ \\
\hline CXCL11 & -0.83891 & 0.005151 & 0.005151 & -2.99784 & Down & $\mathrm{C}-\mathrm{X}-\mathrm{C}$ motif chemokine ligand 11 \\
\hline
\end{tabular}


Table 1 (continued)

\begin{tabular}{|c|c|c|c|c|c|c|}
\hline Gene Symbol & $\log \mathrm{FC}$ & pValue & adj.P.Val & t value & Regulation & Gene Name \\
\hline MASP1 & -0.50677 & 0.005182 & 0.005182 & -2.99543 & Down & Mannan binding lectin serine peptidase 1 \\
\hline MAP4K4 & -0.37777 & 0.005449 & 0.005449 & -2.97583 & Down & $\begin{array}{l}\text { Mitogen-activated protein kinase kinaseki- } \\
\text { nasekinase } 4\end{array}$ \\
\hline CX3CL1 & -0.50445 & 0.005597 & 0.005597 & -2.96535 & Down & $\mathrm{C}-\mathrm{X} 3-\mathrm{C}$ motif chemokine ligand 1 \\
\hline BATF3 & -0.62586 & 0.005862 & 0.005862 & -2.94725 & Down & $\begin{array}{l}\text { Basic leucine zipper ATF-like transcription } \\
\text { factor } 3\end{array}$ \\
\hline CCR8 & -0.51627 & 0.005946 & 0.005946 & -2.94164 & Down & $\mathrm{C}-\mathrm{C}$ motif chemokine receptor 8 \\
\hline TAL1 & -1.01455 & 0.006016 & 0.006016 & -2.93707 & Down & $\begin{array}{l}\text { TAL bHLH transcription factor 1, erythroid } \\
\text { differentiation factor }\end{array}$ \\
\hline NFIL3 & -0.723 & 0.007 & 0.007 & -2.87725 & Down & Nuclear factor, interleukin 3 regulated \\
\hline CD8A & -0.50595 & 0.007337 & 0.007337 & -2.85858 & Down & CD8a molecule \\
\hline CLEC6A & -0.62323 & 0.007445 & 0.007445 & -2.85274 & Down & C-type lectin domain containing $6 \mathrm{~A}$ \\
\hline TCF4 & -0.37827 & 0.007904 & 0.007904 & -2.8289 & Down & Transcription factor 4 \\
\hline FCGR2A & -0.664 & 0.009089 & 0.009089 & -2.77283 & Down & Fc fragment of IgG receptor IIa \\
\hline HLA-B & -0.31677 & 0.00918 & 0.00918 & -2.76877 & Down & major histocompatibility complex, class I, B \\
\hline IRF8 & -0.39477 & 0.009346 & 0.009346 & -2.76157 & Down & Interferon regulatory factor 8 \\
\hline MAPK11 & -0.60427 & 0.010152 & 0.010152 & -2.72804 & Down & Mitogen-activated protein kinase 11 \\
\hline ILF3 & -0.17055 & 0.010366 & 0.010366 & -2.71958 & Down & Interleukin enhancer binding factor 3 \\
\hline XCR1 & -0.34541 & 0.01065 & 0.01065 & -2.70856 & Down & $\mathrm{X}-\mathrm{C}$ motif chemokine receptor 1 \\
\hline ITGAE & -0.33509 & 0.011424 & 0.011424 & -2.67988 & Down & Integrin subunit alpha E \\
\hline IL4R & -0.69064 & 0.012538 & 0.012538 & -2.64162 & Down & Interleukin 4 receptor \\
\hline CTSC & -0.16718 & 0.012619 & 0.012619 & -2.63897 & Down & Cathepsin C \\
\hline ETS1 & -0.39405 & 0.013255 & 0.013255 & -2.61865 & Down & ETS proto-oncogene 1, transcription factor \\
\hline CFI & -0.30082 & 0.013335 & 0.013335 & -2.61614 & Down & Complement factor I \\
\hline STAT5A & -0.27168 & 0.014802 & 0.014802 & -2.57273 & Down & $\begin{array}{l}\text { Signal transducer and activator of transcrip- } \\
\text { tion } 5 \mathrm{~A}\end{array}$ \\
\hline $\mathrm{C} 8 \mathrm{~A}$ & -0.37964 & 0.016204 & 0.016204 & -2.53478 & Down & Complement C8 alpha chain \\
\hline DEFB4A & -0.36691 & 0.017835 & 0.017835 & -2.49428 & Down & Defensin beta $4 \mathrm{~A}$ \\
\hline RELB & -0.45095 & 0.019351 & 0.019351 & -2.45958 & Down & RELB proto-oncogene, NF-kB subunit \\
\hline ATG7 & -0.32859 & 0.01984 & 0.01984 & -2.4489 & Down & Autophagy related 7 \\
\hline DPP4 & -0.38823 & 0.01999 & 0.01999 & -2.44568 & Down & Dipeptidyl peptidase 4 \\
\hline GPI & -0.21382 & 0.020424 & 0.020424 & -2.43646 & Down & Glucose-6-phosphate isomerase \\
\hline CD59 & -0.24032 & 0.020561 & 0.020561 & -2.43359 & Down & CD59 molecule (CD59 blood group) \\
\hline CASP3 & -0.40573 & 0.025629 & 0.025629 & -2.33807 & Down & Caspase 3 \\
\hline TIGIT & -0.3615 & 0.025963 & 0.025963 & -2.33239 & Down & $\begin{array}{l}\text { T cell immunoreceptor with Ig and ITIM } \\
\text { domains }\end{array}$ \\
\hline CFD & -0.36627 & 0.026073 & 0.026073 & -2.33053 & Down & Complement factor D \\
\hline CCL18 & -0.42077 & 0.026944 & 0.026944 & -2.3161 & Down & $\mathrm{C}-\mathrm{C}$ motif chemokine ligand 18 \\
\hline PLAU & -0.34086 & 0.028132 & 0.028132 & -2.29706 & Down & Plasminogen activator, urokinase \\
\hline PTPN22 & -0.20086 & 0.028829 & 0.028829 & -2.28623 & Down & $\begin{array}{l}\text { Protein tyrosine phosphatase non-receptor } \\
\text { type } 22\end{array}$ \\
\hline TOLLIP & -0.27377 & 0.028911 & 0.028911 & -2.28497 & Down & Toll interacting protein \\
\hline CXCR2 & -0.49195 & 0.029438 & 0.029438 & -2.27696 & Down & C-X-C motif chemokine receptor 2 \\
\hline CCL20 & -0.34555 & 0.029871 & 0.029871 & -2.27048 & Down & $\mathrm{C}-\mathrm{C}$ motif chemokine ligand 20 \\
\hline IL12A & -0.40759 & 0.03046 & 0.03046 & -2.26179 & Down & Interleukin $12 \mathrm{~A}$ \\
\hline IL10 & -0.33555 & 0.03205 & 0.03205 & -2.23904 & Down & Interleukin 10 \\
\hline LILRA1 & -0.26991 & 0.033542 & 0.033542 & -2.21862 & Down & leukocyte immunoglobulin like receptor A1 \\
\hline PTPN6 & -0.16109 & 0.034722 & 0.034722 & -2.20304 & Down & $\begin{array}{l}\text { protein tyrosine phosphatase non-receptor } \\
\text { type } 6\end{array}$ \\
\hline CCL19 & -0.37718 & 0.035605 & 0.035605 & -2.19168 & Down & $\mathrm{C}-\mathrm{C}$ motif chemokine ligand 19 \\
\hline IKBKAP & -0.15505 & 0.035624 & 0.035624 & -2.19144 & Down & IкB kinase complex-associated protein \\
\hline
\end{tabular}


Table 1 (continued)

\begin{tabular}{|c|c|c|c|c|c|c|}
\hline Gene Symbol & $\log \mathrm{FC}$ & pValue & adj.P.Val & $\mathrm{t}$ value & Regulation & Gene Name \\
\hline TNFRSF9 & -0.50786 & 0.036235 & 0.036235 & -2.18372 & Down & TNF receptor superfamily member 9 \\
\hline TCF7 & -0.60091 & 0.036564 & 0.036564 & -2.17963 & Down & Transcription factor 7 \\
\hline HLA-DMA & -0.30777 & 0.036925 & 0.036925 & -2.17516 & Down & $\begin{array}{l}\text { Major histocompatibility complex, class II, } \\
\text { DM alpha }\end{array}$ \\
\hline CXCL12 & -0.69291 & 0.037168 & 0.037168 & -2.17218 & Down & $\mathrm{C}-\mathrm{X}-\mathrm{C}$ motif chemokine ligand 12 \\
\hline MBL2 & -0.37009 & 0.037623 & 0.037623 & -2.16664 & Down & Mannose binding lectin 2 \\
\hline IKZF2 & -0.46655 & 0.039489 & 0.039489 & -2.14452 & Down & IKAROS family zinc finger 2 \\
\hline BCAP31 & -0.10632 & 0.04075 & 0.04075 & -2.13009 & Down & B cell receptor associated protein 31 \\
\hline HLA-DRB1 & -1.39495 & 0.041403 & 0.041403 & -2.12278 & Down & $\begin{array}{l}\text { Major histocompatibility complex, class II, } \\
\text { DR beta } 1\end{array}$ \\
\hline IRAK4 & -0.23727 & 0.041428 & 0.041428 & -2.12251 & Down & Interleukin 1 receptor associated kinase 4 \\
\hline CXCR1 & -0.49741 & 0.041833 & 0.041833 & -2.11802 & Down & $\mathrm{C}-\mathrm{X}-\mathrm{C}$ motif chemokine receptor 1 \\
\hline PTK2 & -0.33609 & 0.043242 & 0.043242 & -2.10272 & Down & Protein tyrosine kinase 2 \\
\hline CD45R0 & -0.46514 & 0.043373 & 0.043373 & -2.10132 & Down & $\begin{array}{l}\text { A member of leucocyte common antigen } \\
\text { family }\end{array}$ \\
\hline
\end{tabular}

phenylalanine tyrosine and tryptophan biosynthesis, MAP kinase activity, genes encoding secreted soluble factors, cytokine network, interleukin signaling pathway, inflammation mediated by chemokine and cytokine signaling pathway, intrinsic apoptotic, interleukin-10 signaling, sulindac pathway, glycolysis and other pathways (Table 3).

\section{Gene ontology (G0) enrichment analysis for DEGs}

The GO enrichment analysis of up- and down-regulated genes can be split into three groups: BP, CC, and MF are listed in Tables 4, 5. In terms of BP, the up-regulated genes were mainly involved in regulation of immune system process, response to biotic stimulus and other functions; the down-regulated genes were mainly associated in regulation of immune system process, cytokine-mediated signaling pathway and other functions. As far as CC is concerned, the up-regulated genes were mainly involved in the side of membrane, receptor complex and other functions; the down-regulated genes were mainly located in the cell surface, leaflet of membrane layers and other functions. As for MF, the up-regulated genes mainly participated in kinase binding, signaling receptor binding and other functions; the down-regulated genes mainly participated in cytokine receptor binding, signaling receptor binding and other functions (Tables 4, 5).

\section{PPI network construction and module analysis}

To determine the expression relationships among up- and down-regulated genes, we inputted the up- and down-regulated genes to STRING PPI database. Then, PPI networks were visualized using the cytoscape software. As a result, a PPI network for up-regulated genes had 2912 nodes and
5967 edges (Fig. 6). Among these nodes, TP53, HRAS, CTNNB1, FYN, ABL1, STAT3, STAT1, JAK2, C1QBP, XBP1, BST2, CD99 and IFI35 were identified as hub genes with highest node degree distribution, betweenness centrality, stress centrality, closeness centrality and lowest clustering coefficient are listed in Table 6. The scatter plots for this network are shown in Fig. 7a-e. Enrichment analysis showed that the genes were mainly associated with measles, natural killer cell mediated cytotoxicity, HTLVI infection, regulation of immune system process, viral myocarditis, Jak-STAT signaling pathway, herpes simplex infection, hemostasis, response to biotic stimulus, cytokine signaling in immune system, integral component of plasma membrane and response to biotic stimulus. A PPI network for down-regulated genes had 3083 nodes and 6491 edges (Fig. 8). Among these nodes, MAPK11, RELA, LCK, KIT, EGR1, IL20, ILF3, CASP3, IL19, ATG7, GPI and S1PR1 were identified as hub genes with highest node degree distribution, betweenness centrality, stress centrality, closeness centrality and lowest clustering coefficient are listed in Table 6. The scatter plots for this network are shown in Fig. 9a-e. Enrichment analysis showed that the genes were mainly associated with tuberculosis, inflammatory bowel disease (IBD), HTLV-I infection, cytokine-cytokine receptor interaction, cytokine-mediated signaling pathway, regulation of immune system process, response to biotic stimulus, response to cytokine, cytokine production, innate immune system, glycolysis, gluconeogenesis and the extracellular signal-regulated RAF/MEK/ERK signaling.

Based on STRING database, plug-ins PEWCC1 was used to carry out module analysis in Cytoscape software. We identified total 566 and 548 modules from the PPI network of up- and down-regulated genes according to the degree of importance and further analyzed with the plug-in 


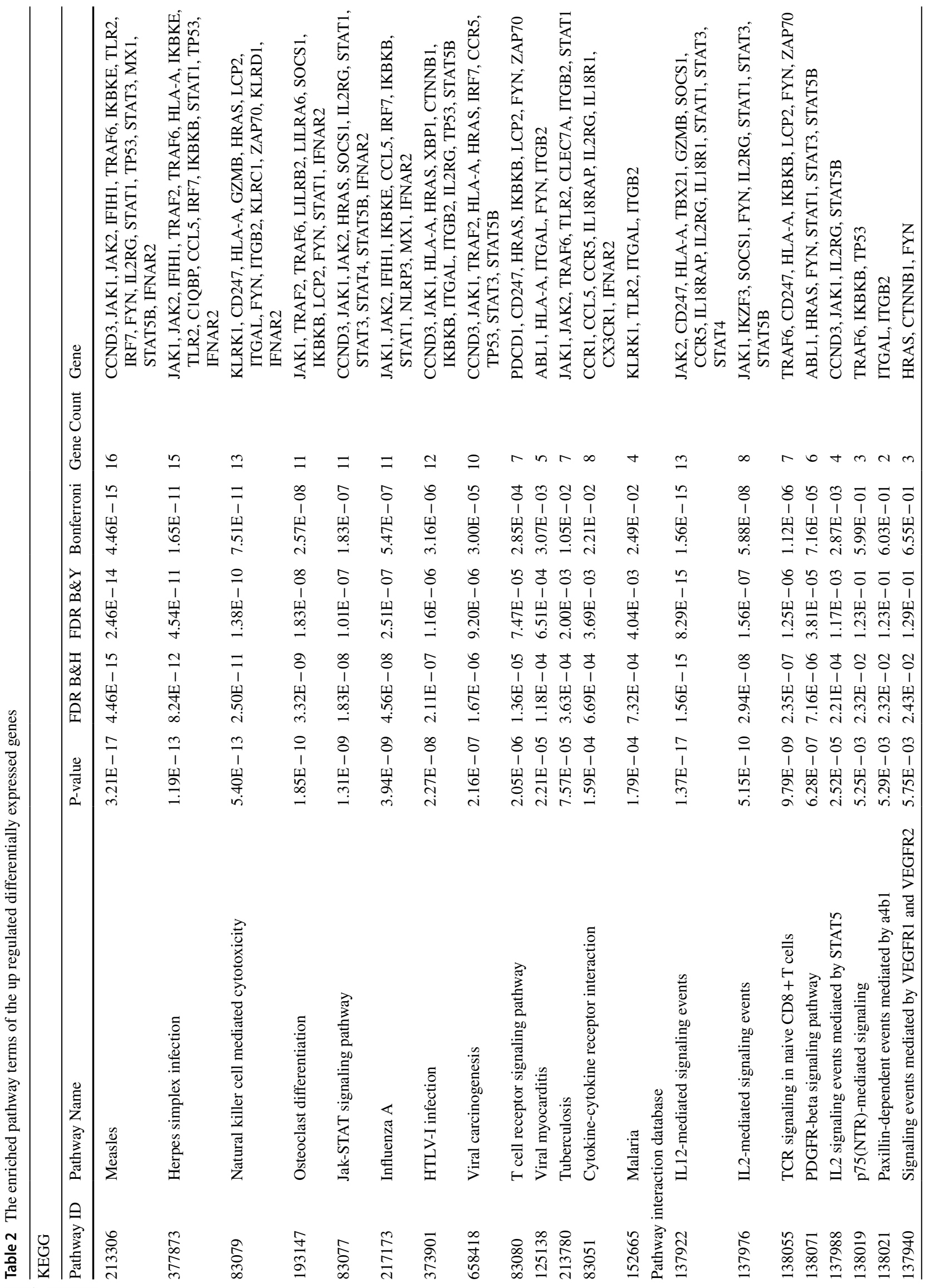




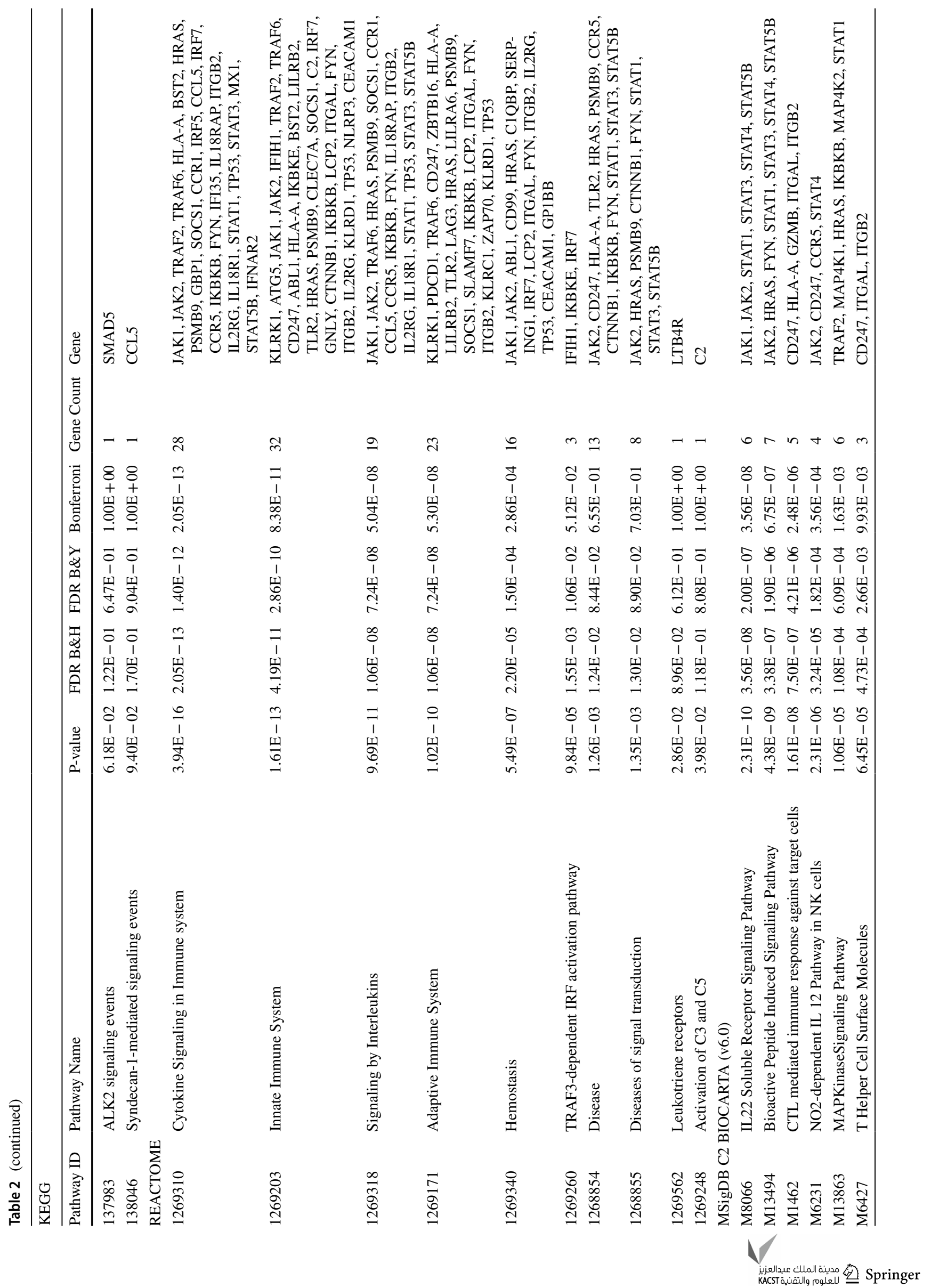




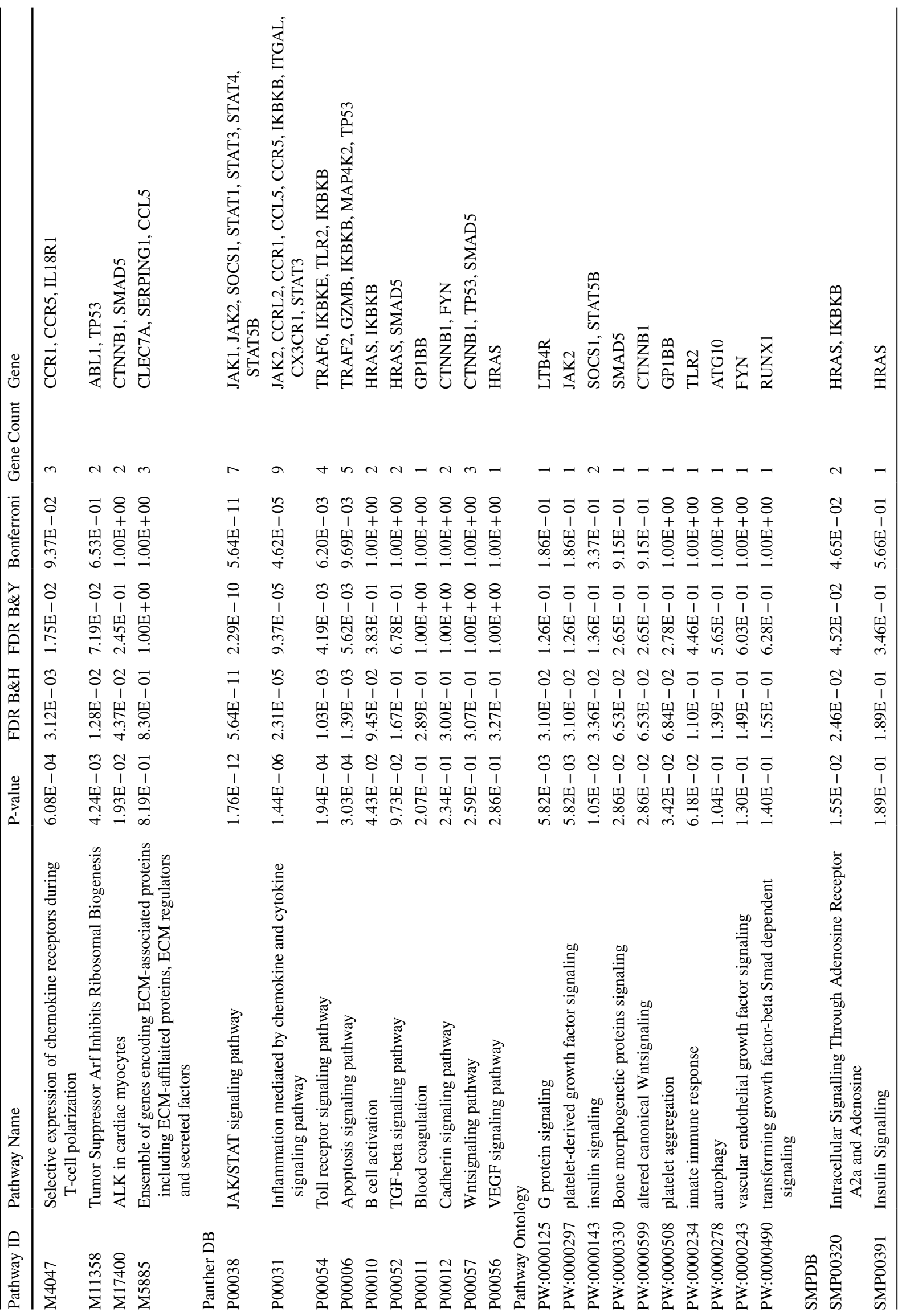




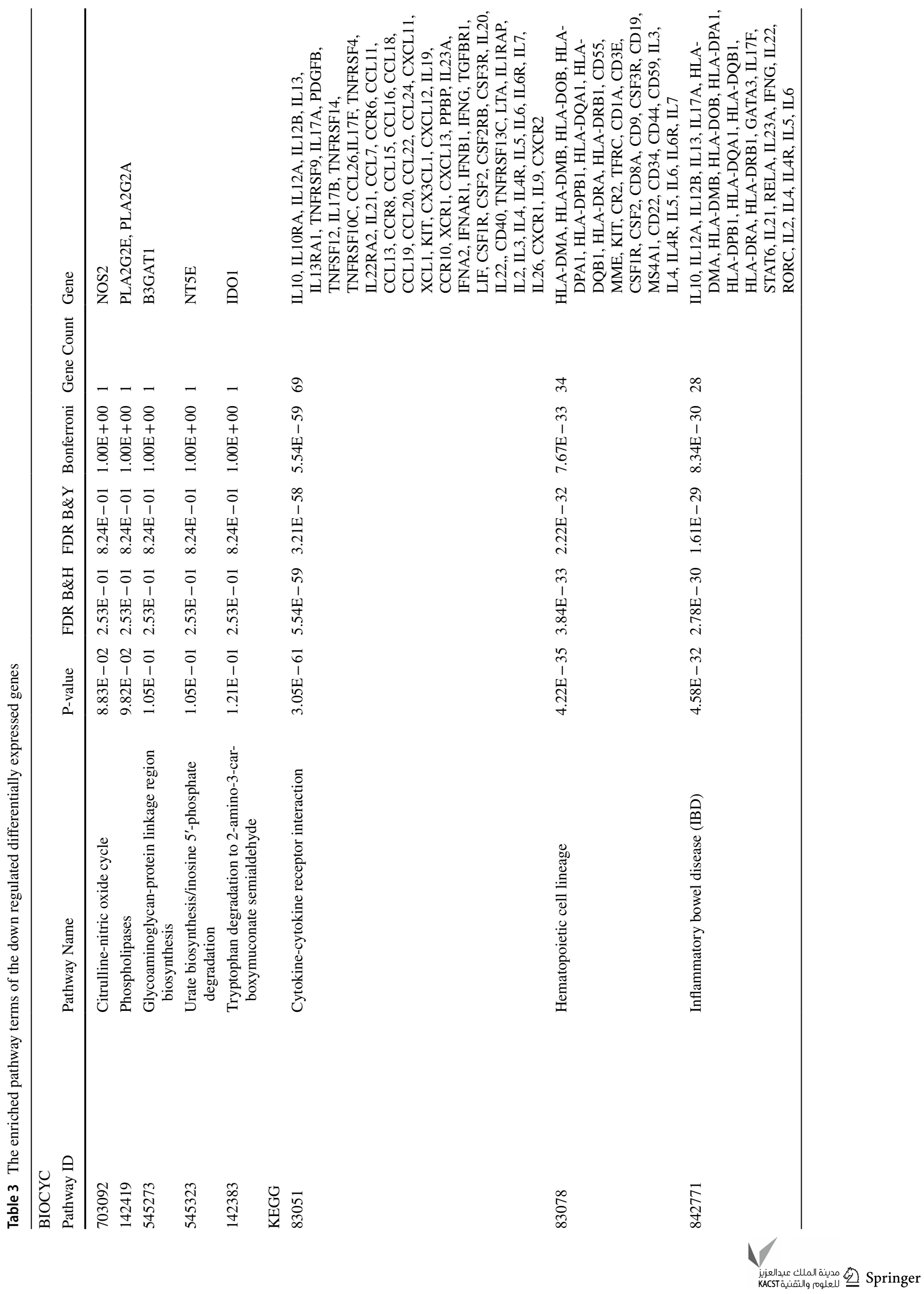




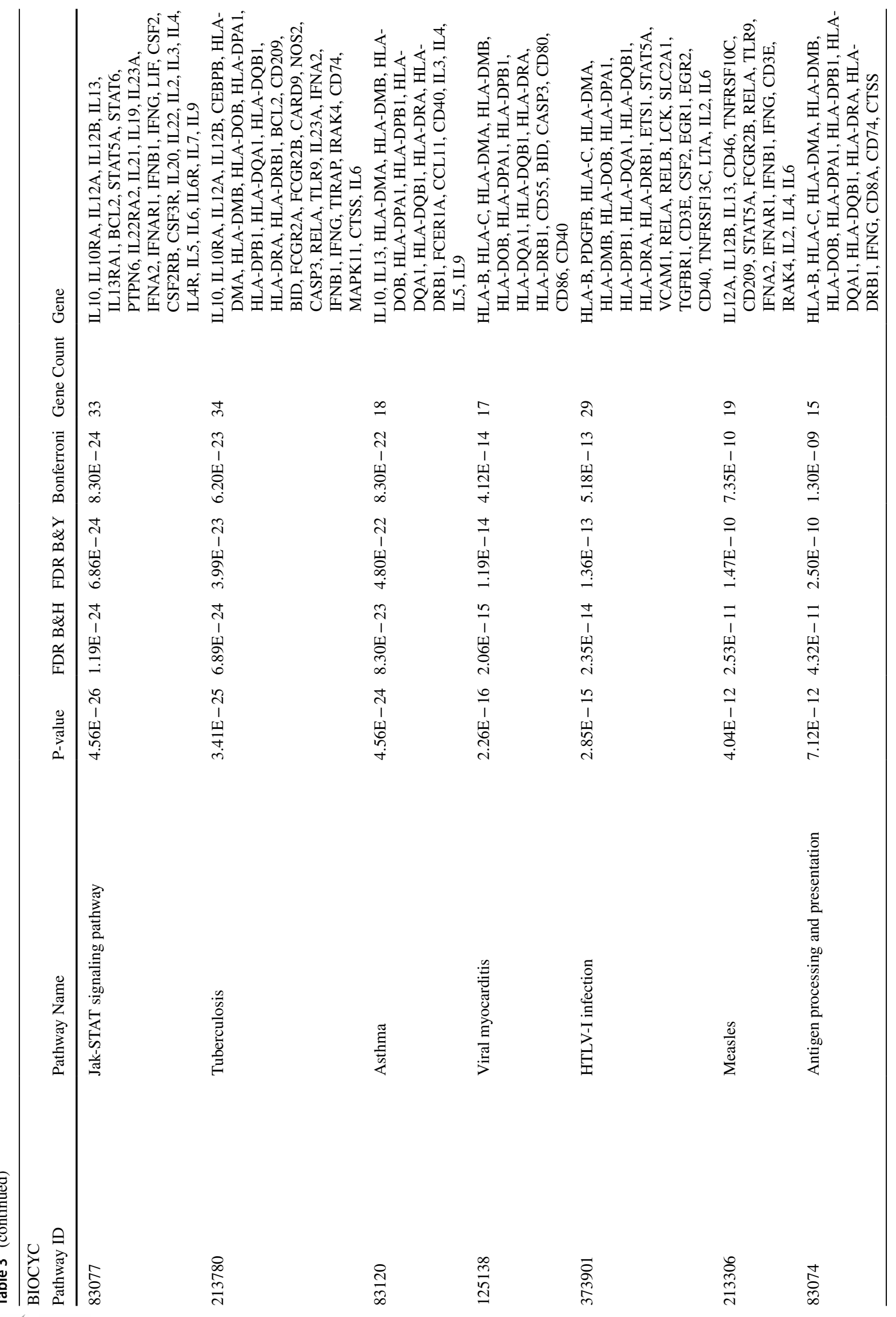




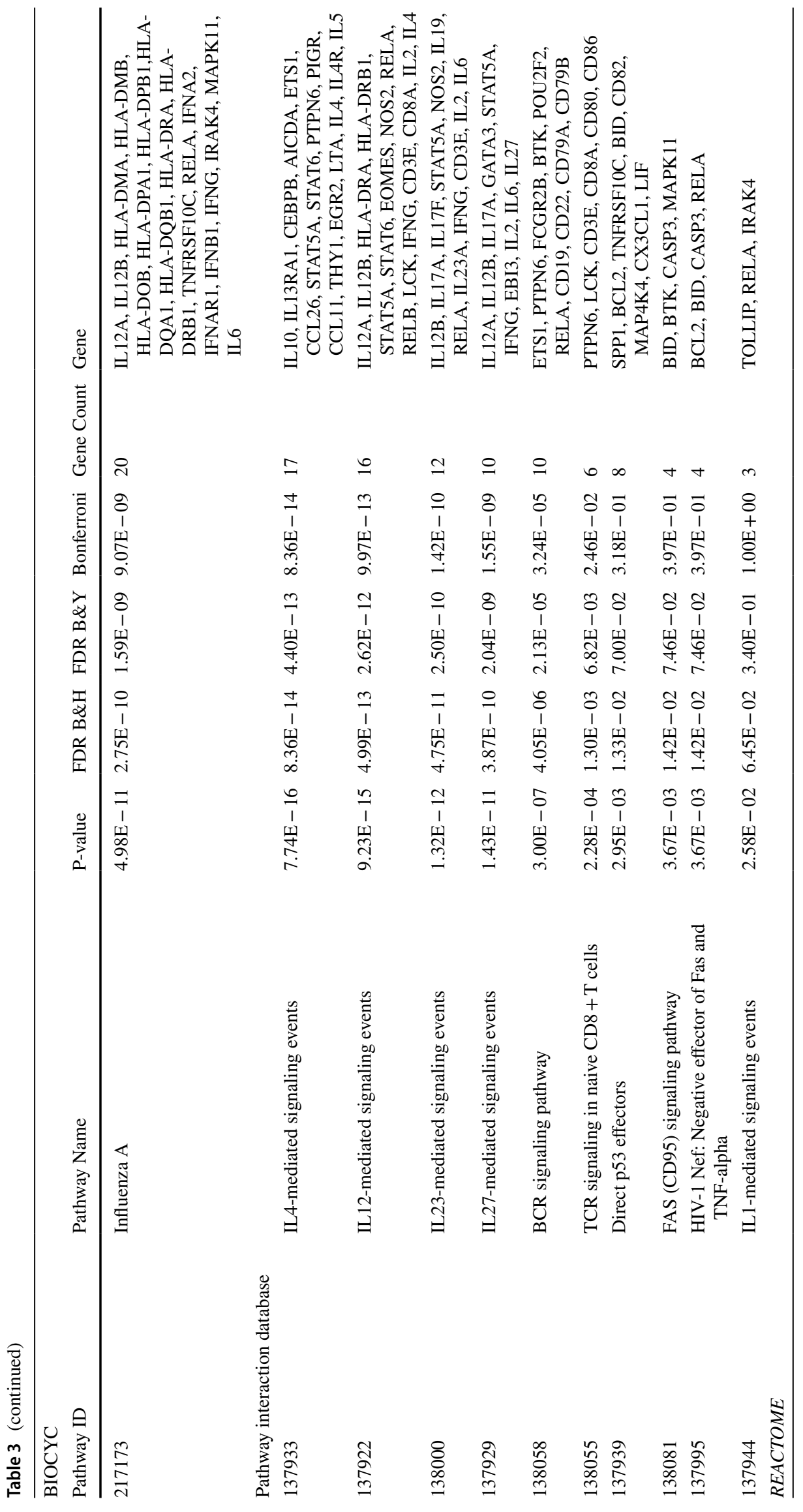


422 Page 20 of 65

3 Biotech (2020) 10:422

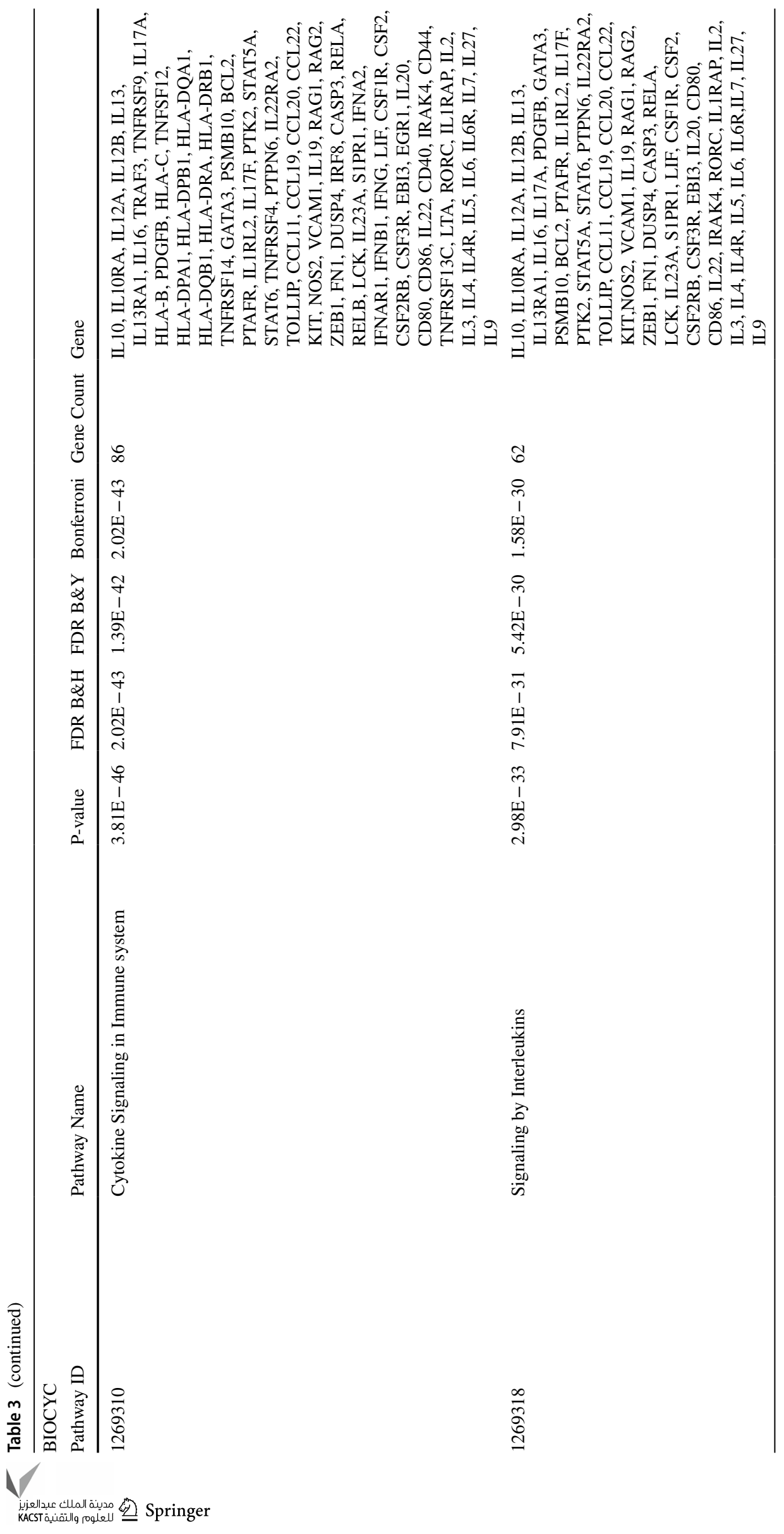




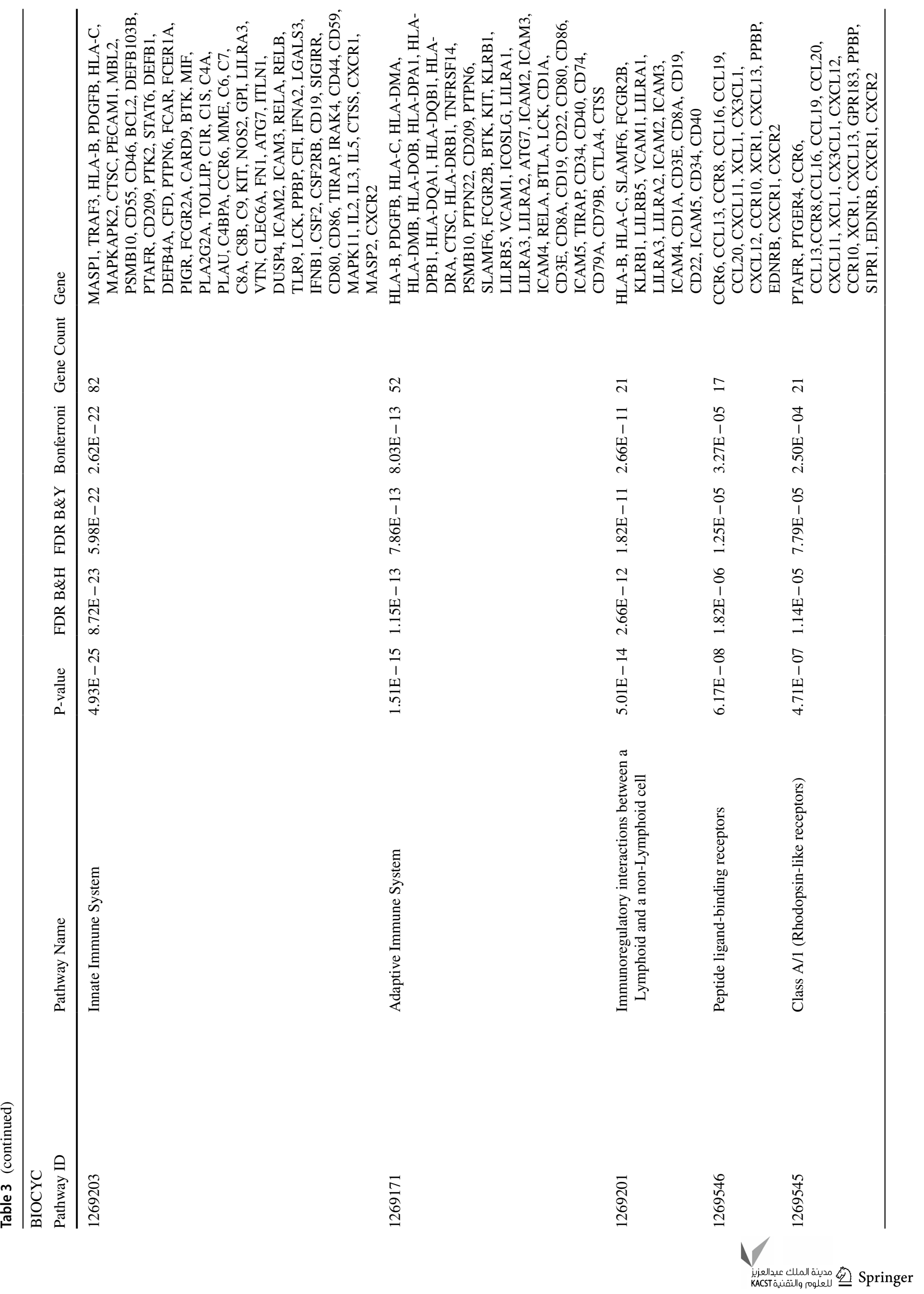




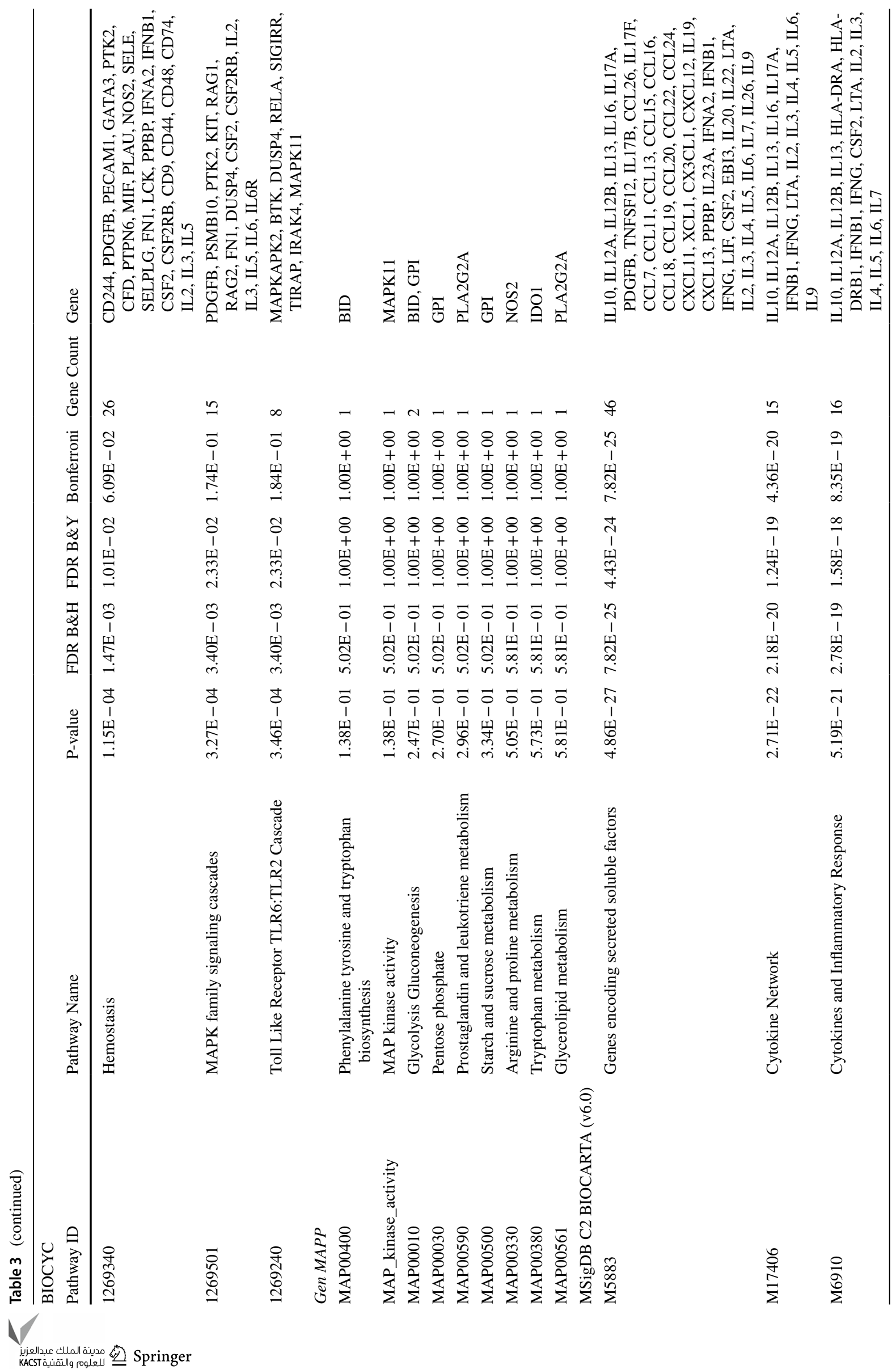




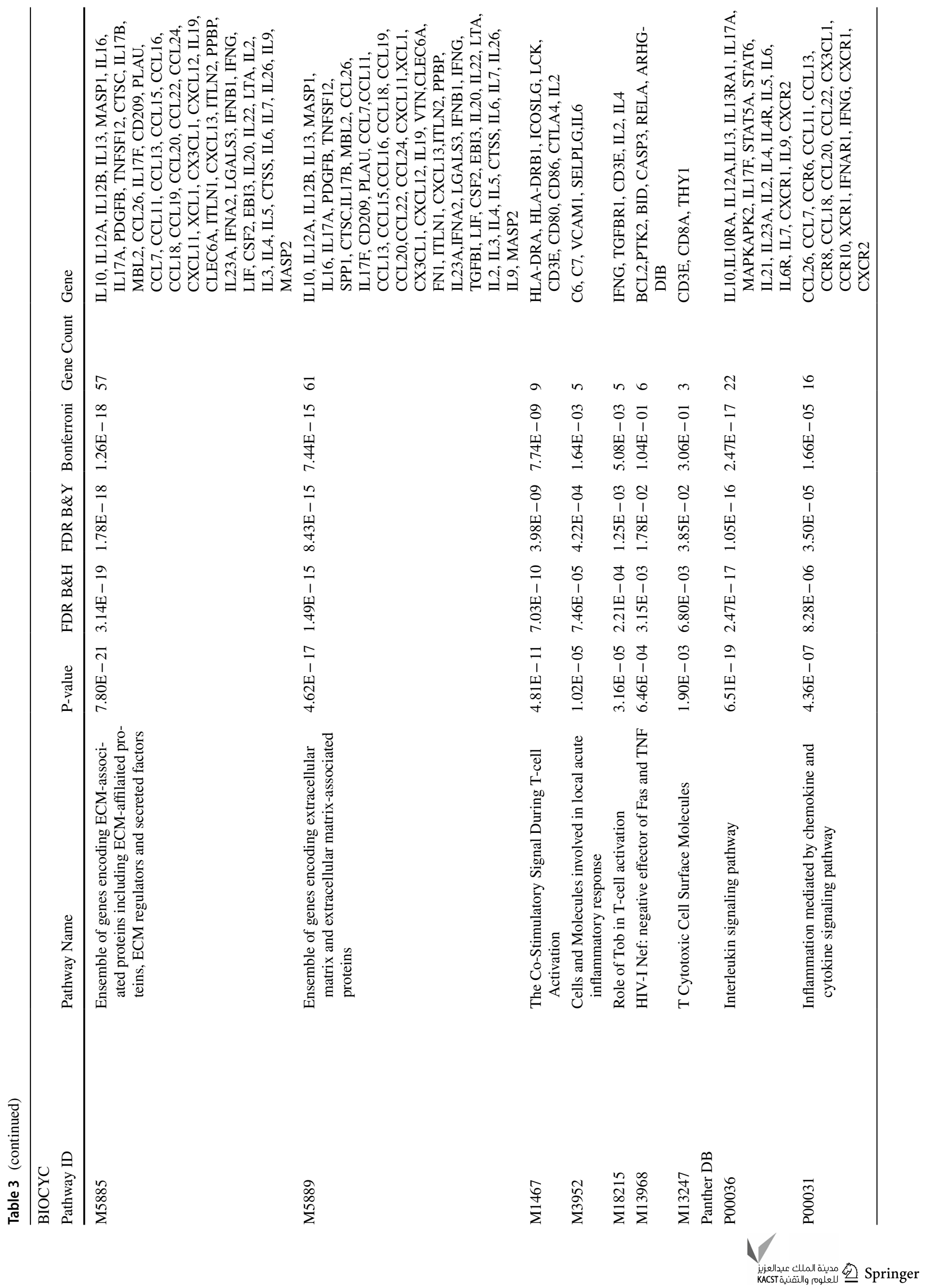




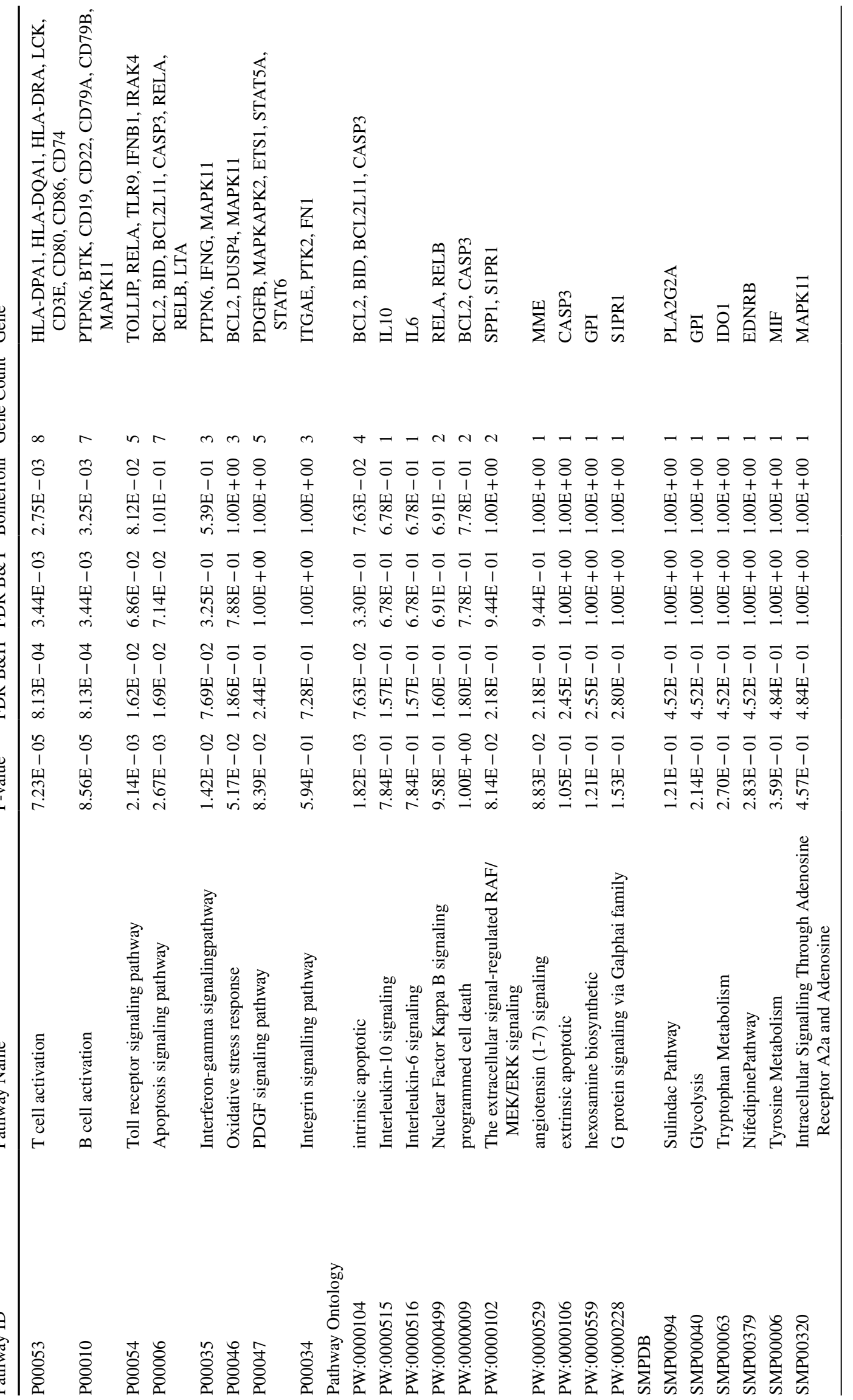




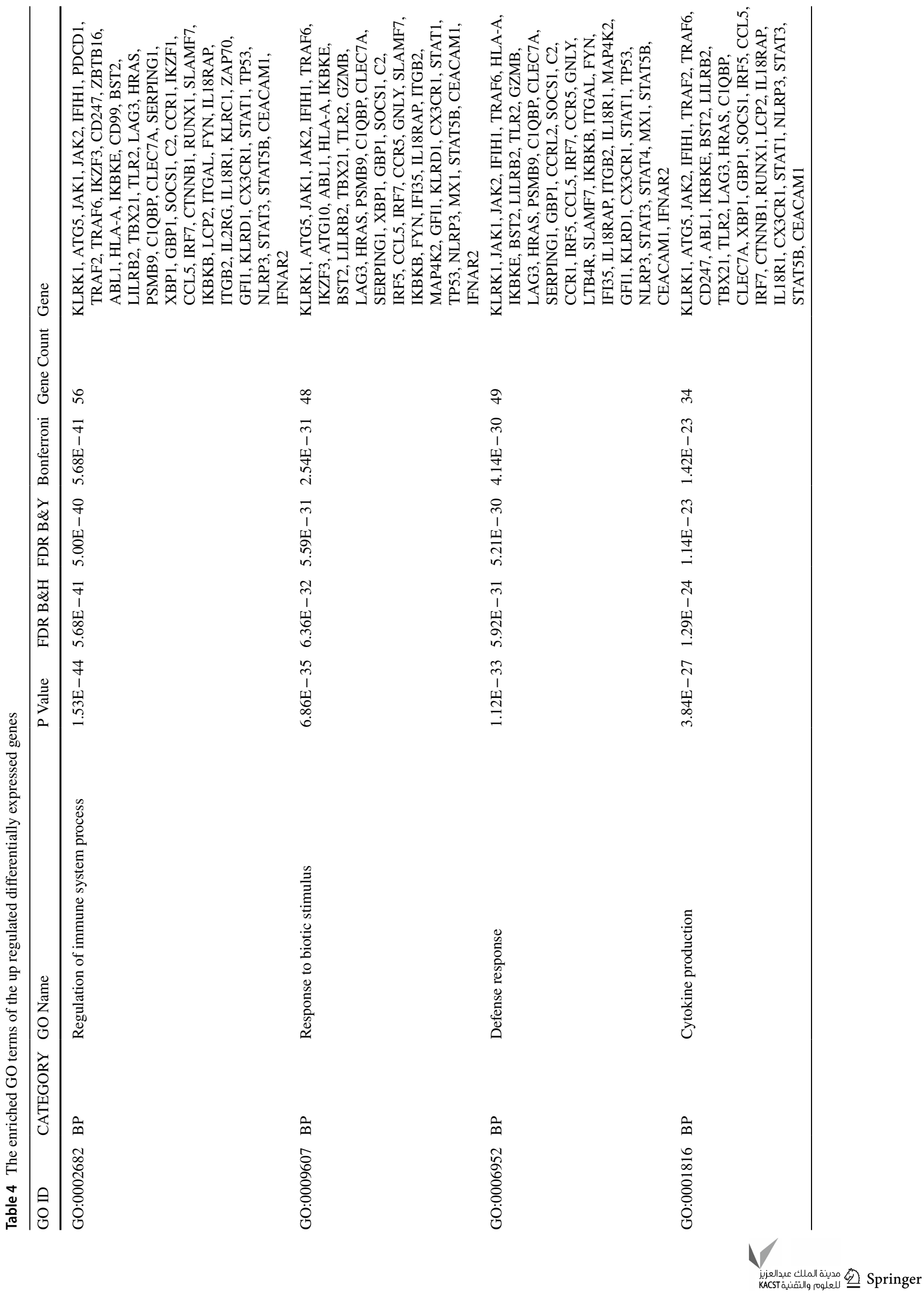




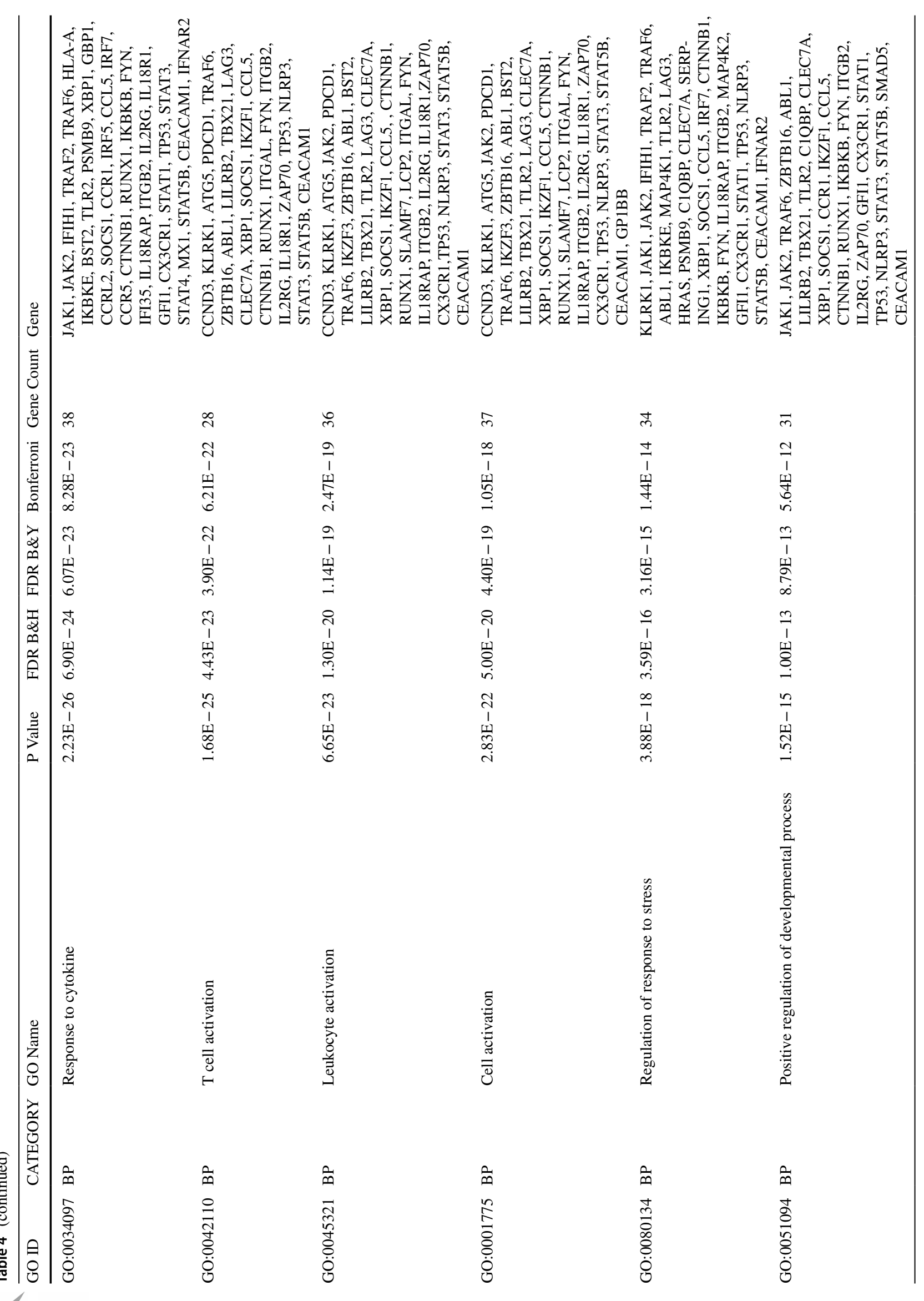




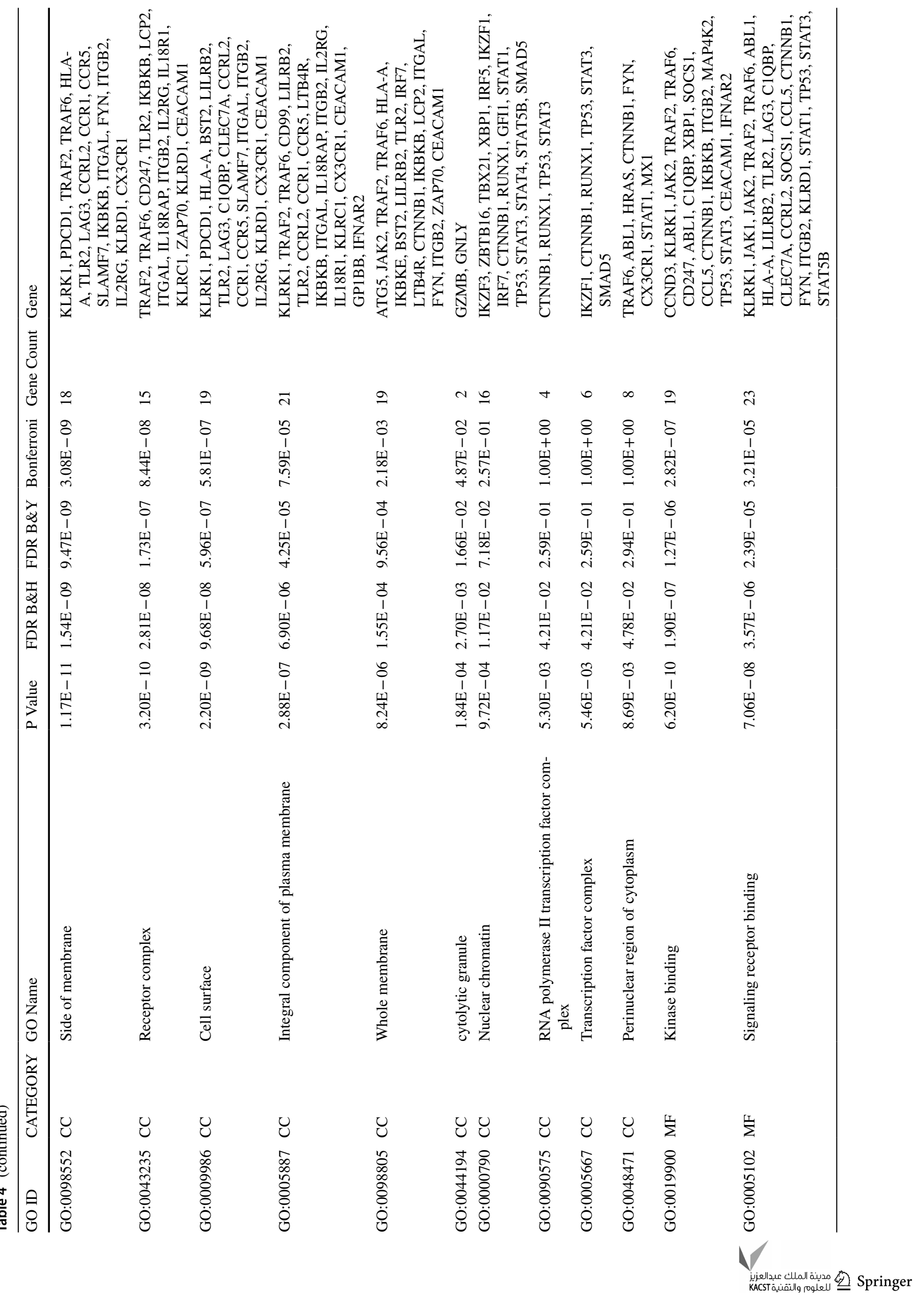




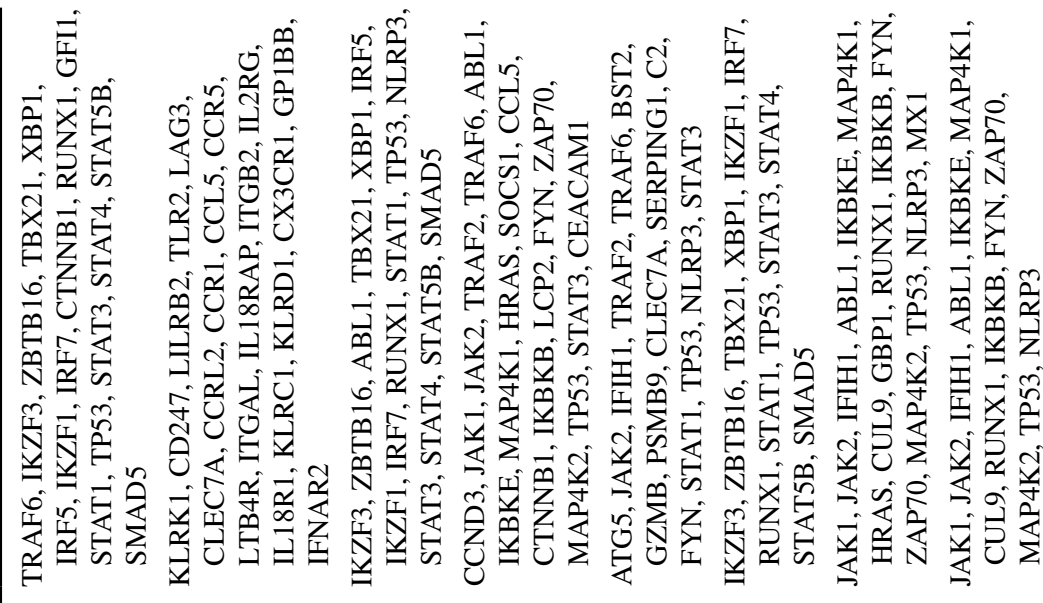

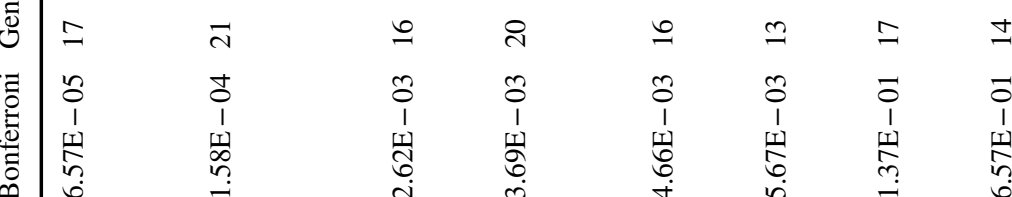

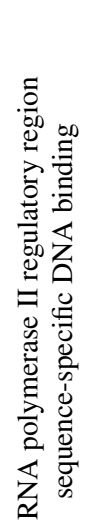

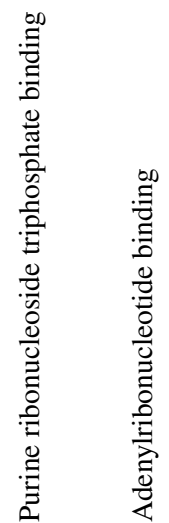


3 Biotech (2020) 10:422

Page 29 of $65 \quad \mathbf{4 2 2}$

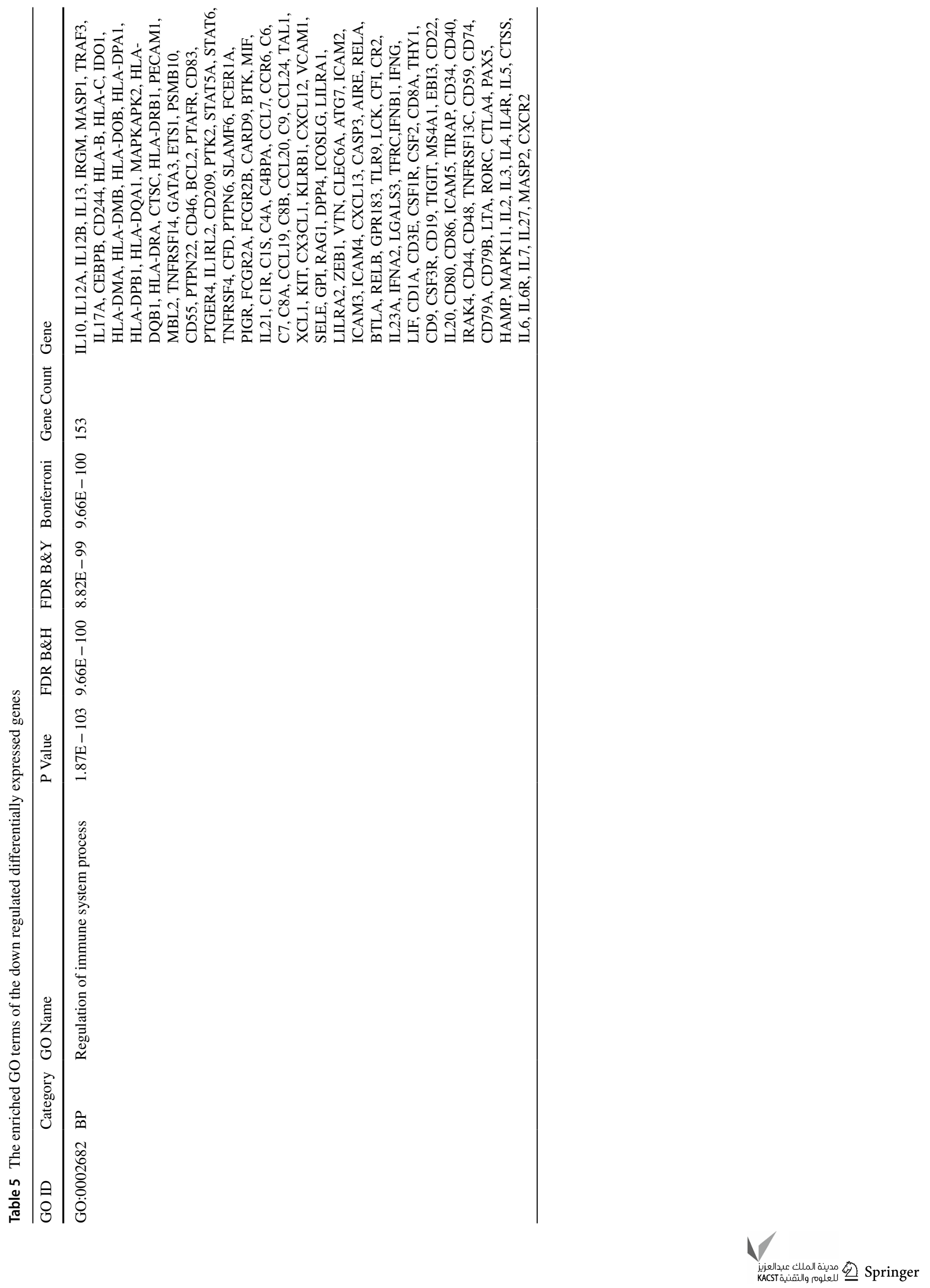




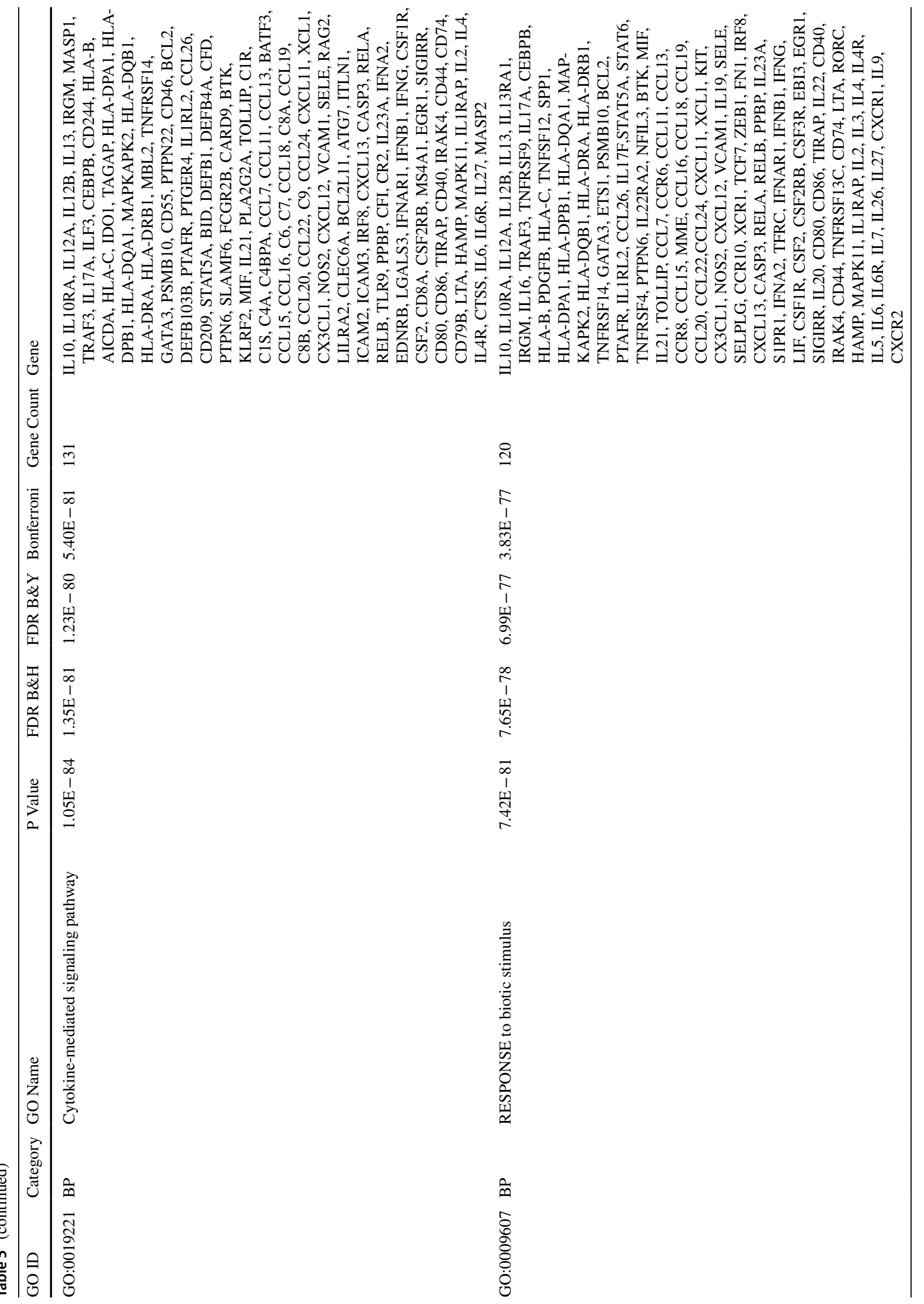




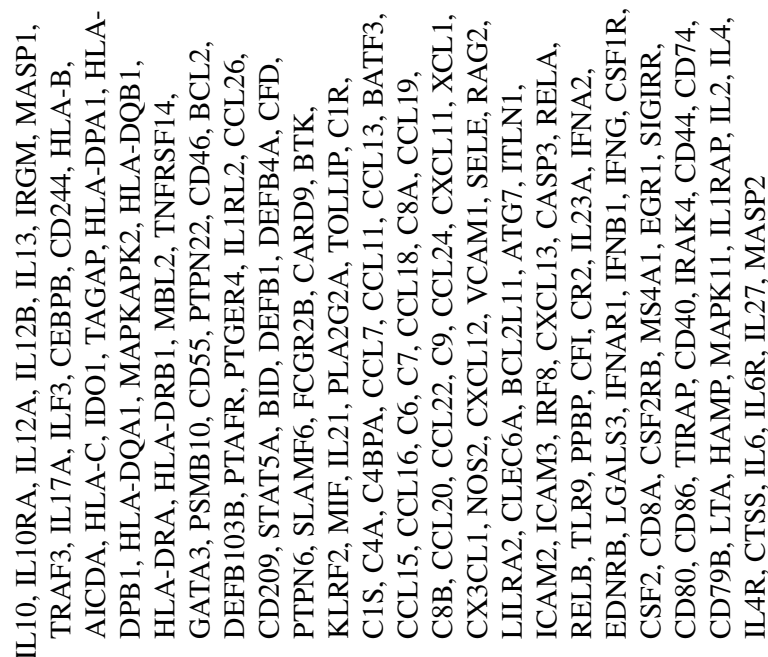

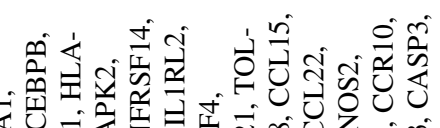
我害官

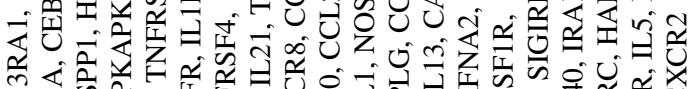

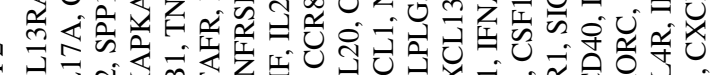

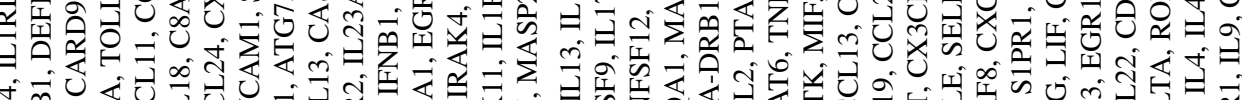

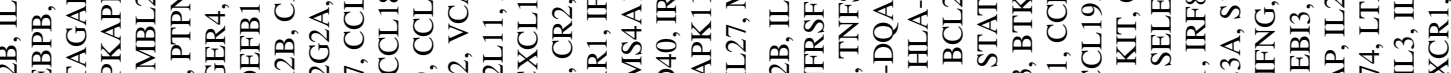

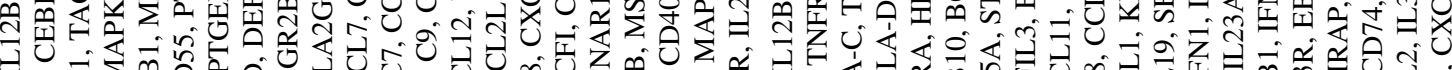

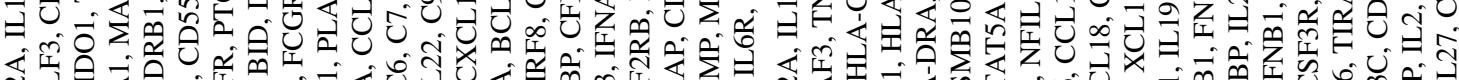

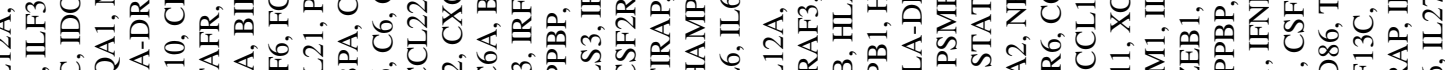

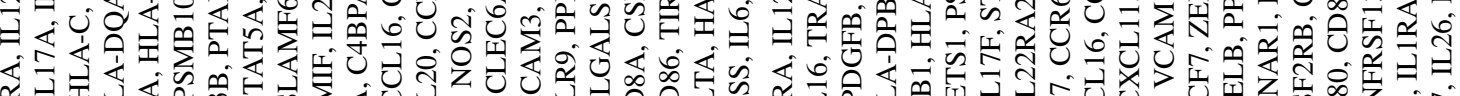

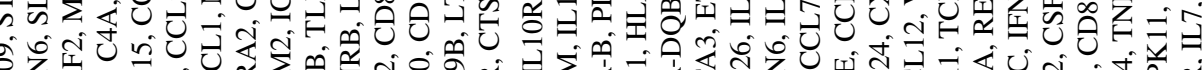

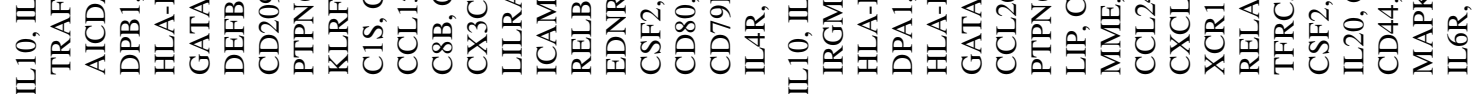

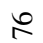

站

$\overline{0}$

떵

딘

$\infty$

岳

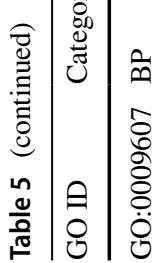

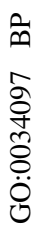

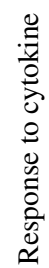




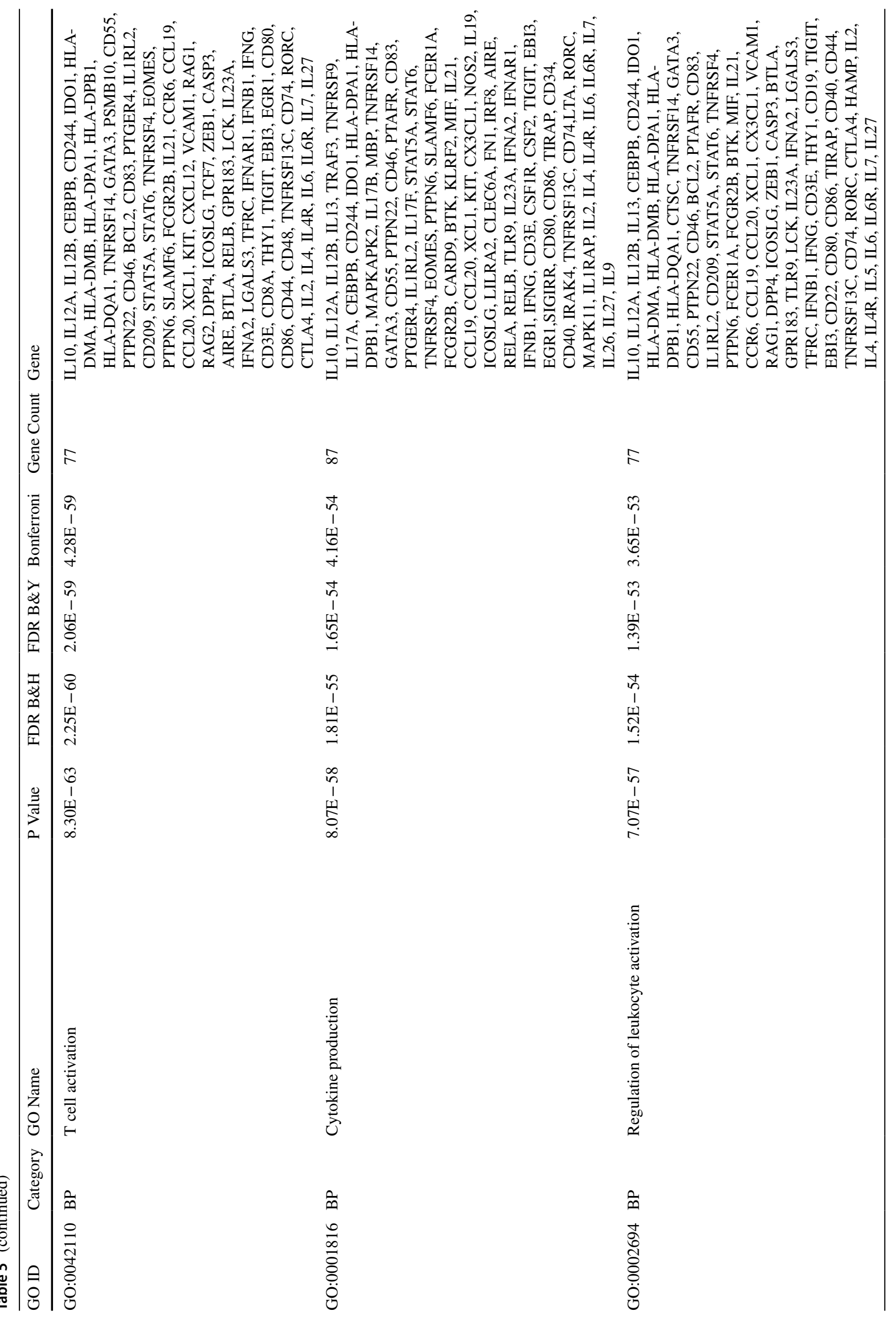


3 Biotech (2020) 10:422

Page 33 of $65 \quad \mathbf{4 2 2}$

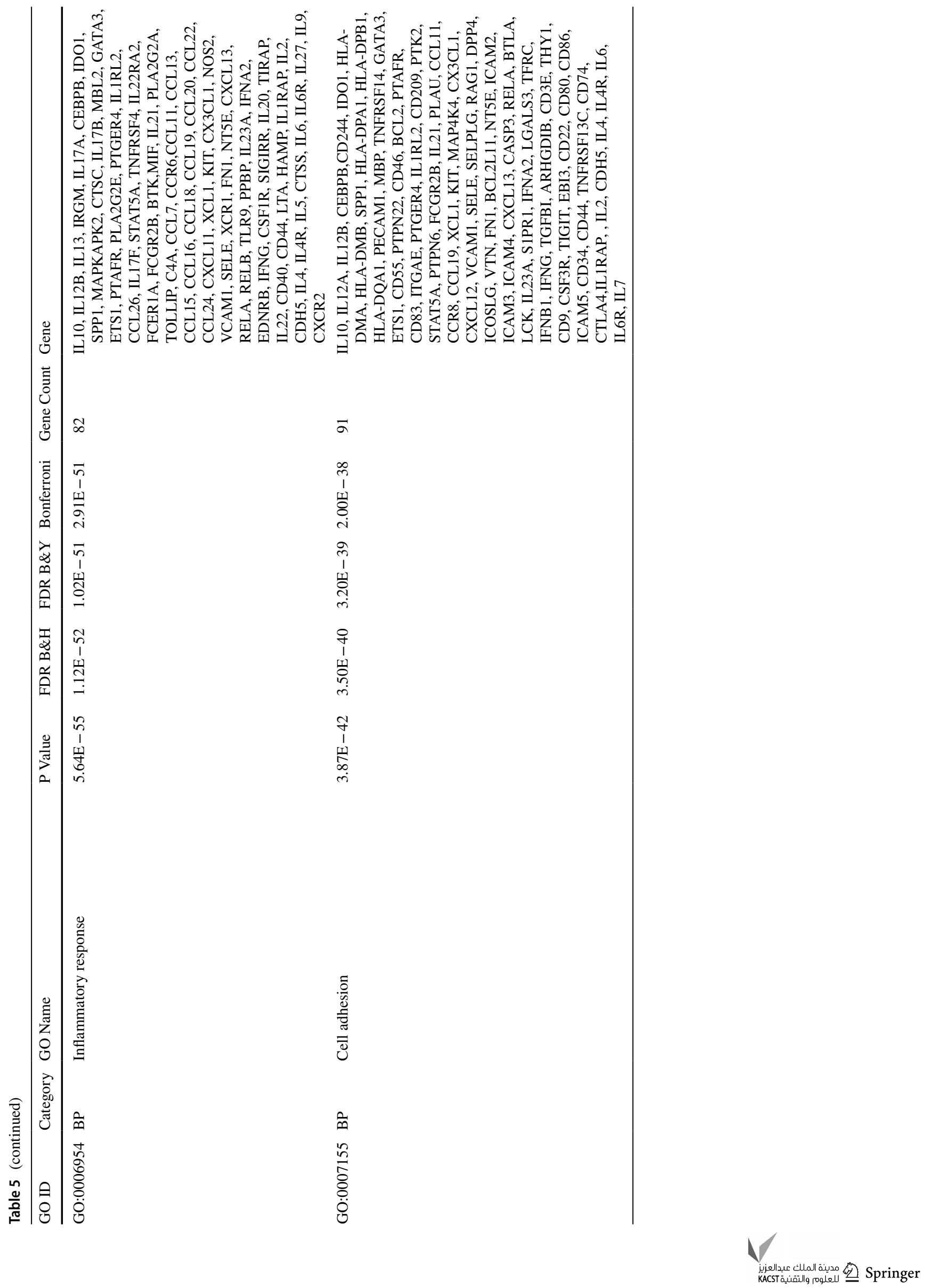




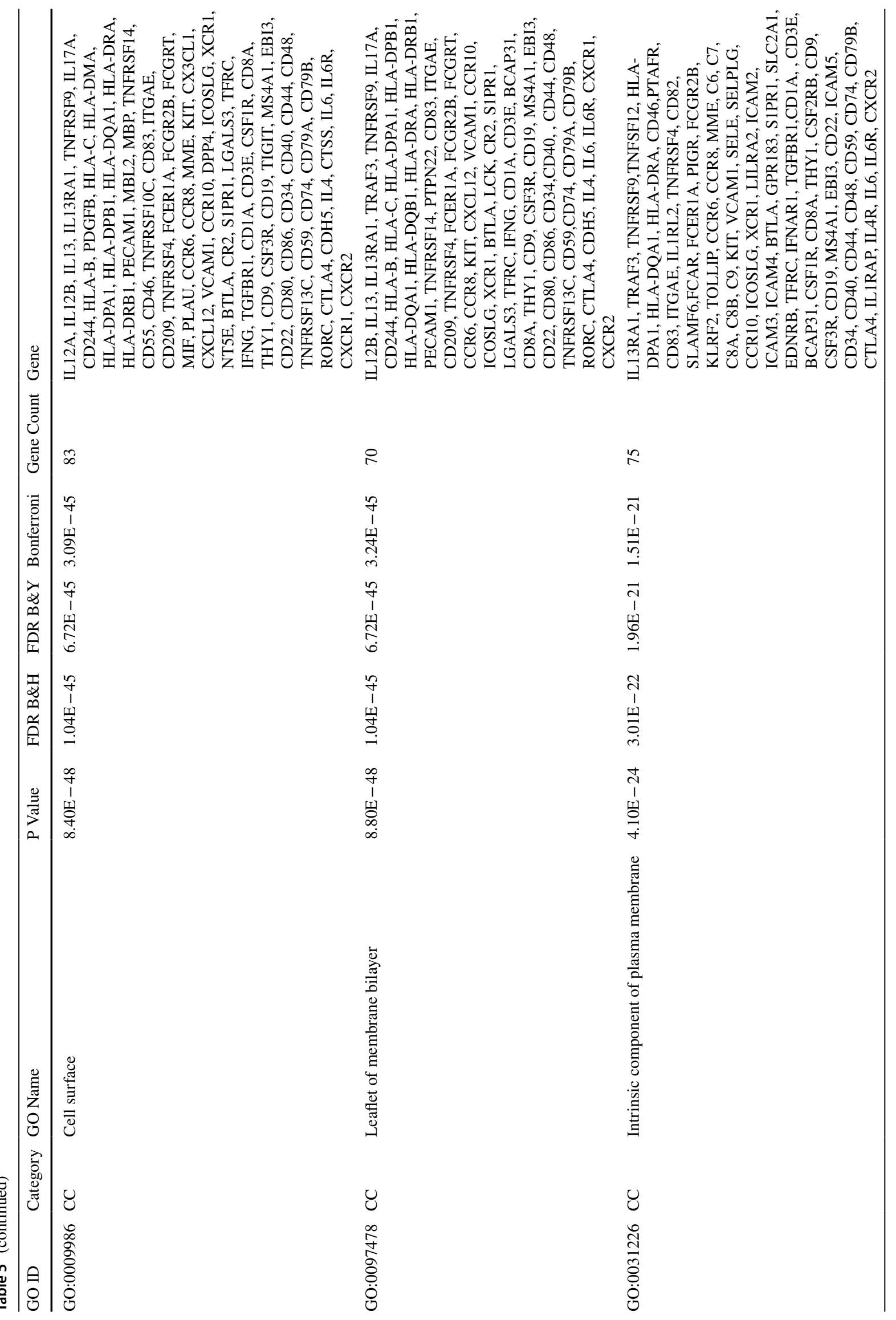




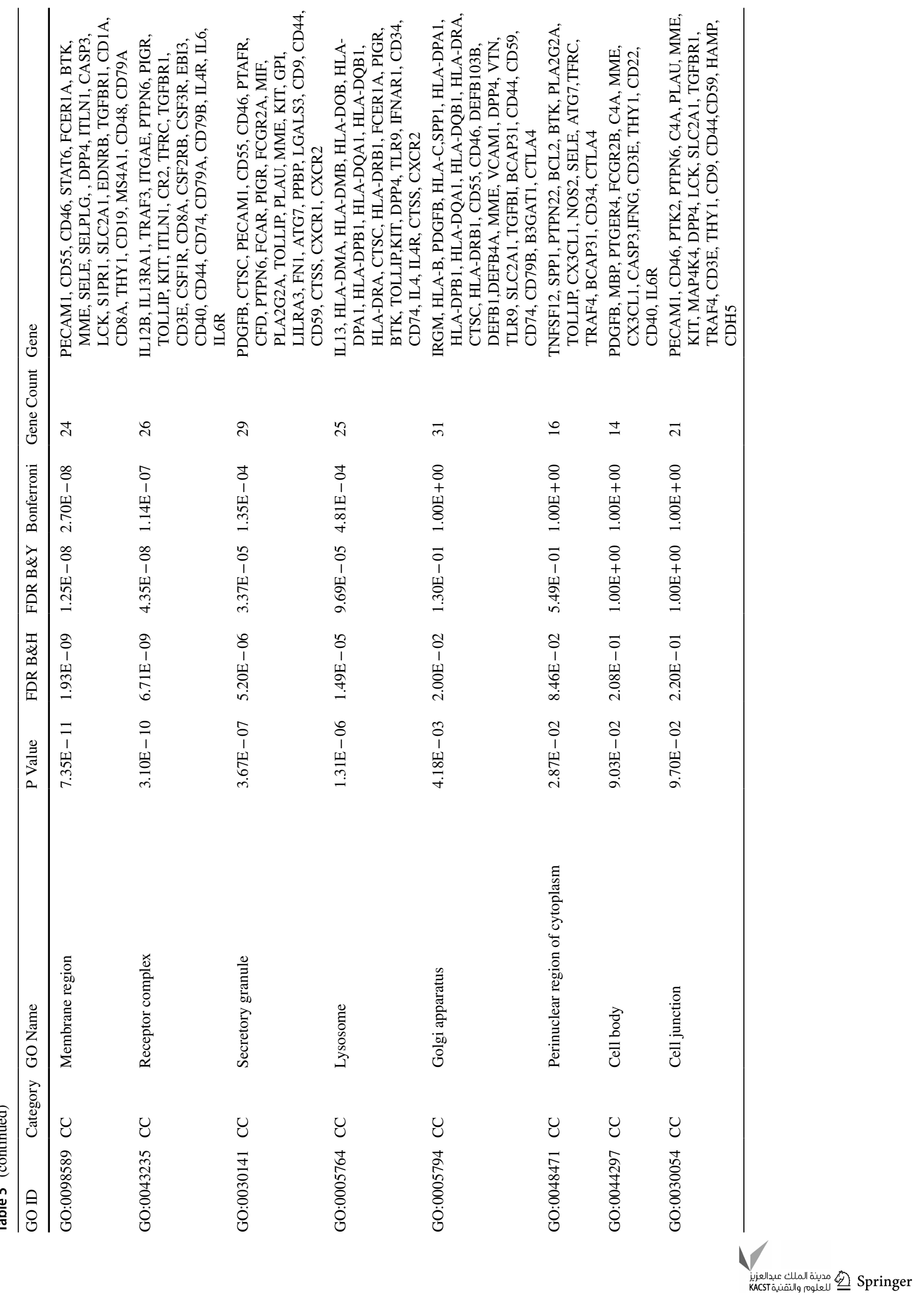




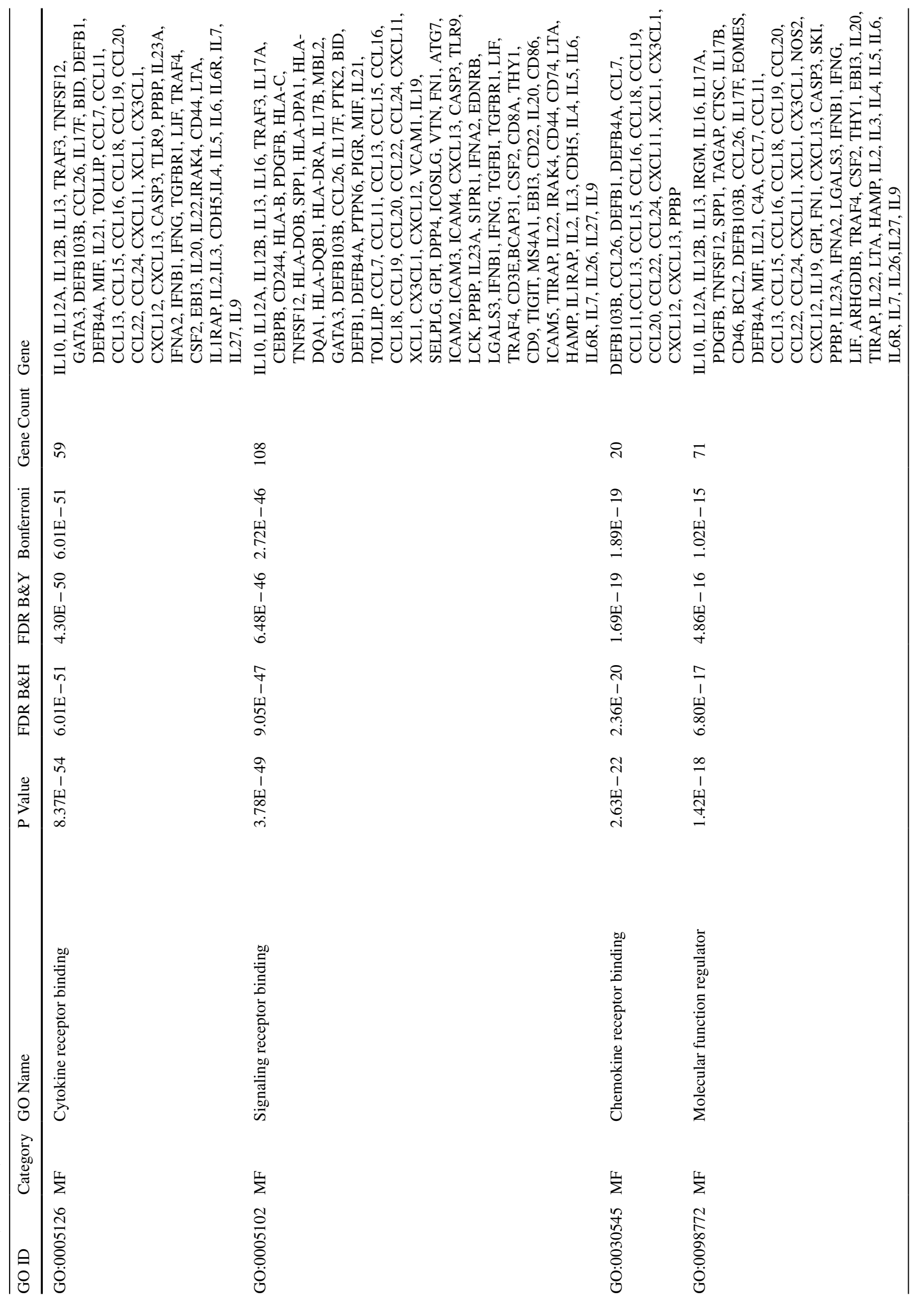




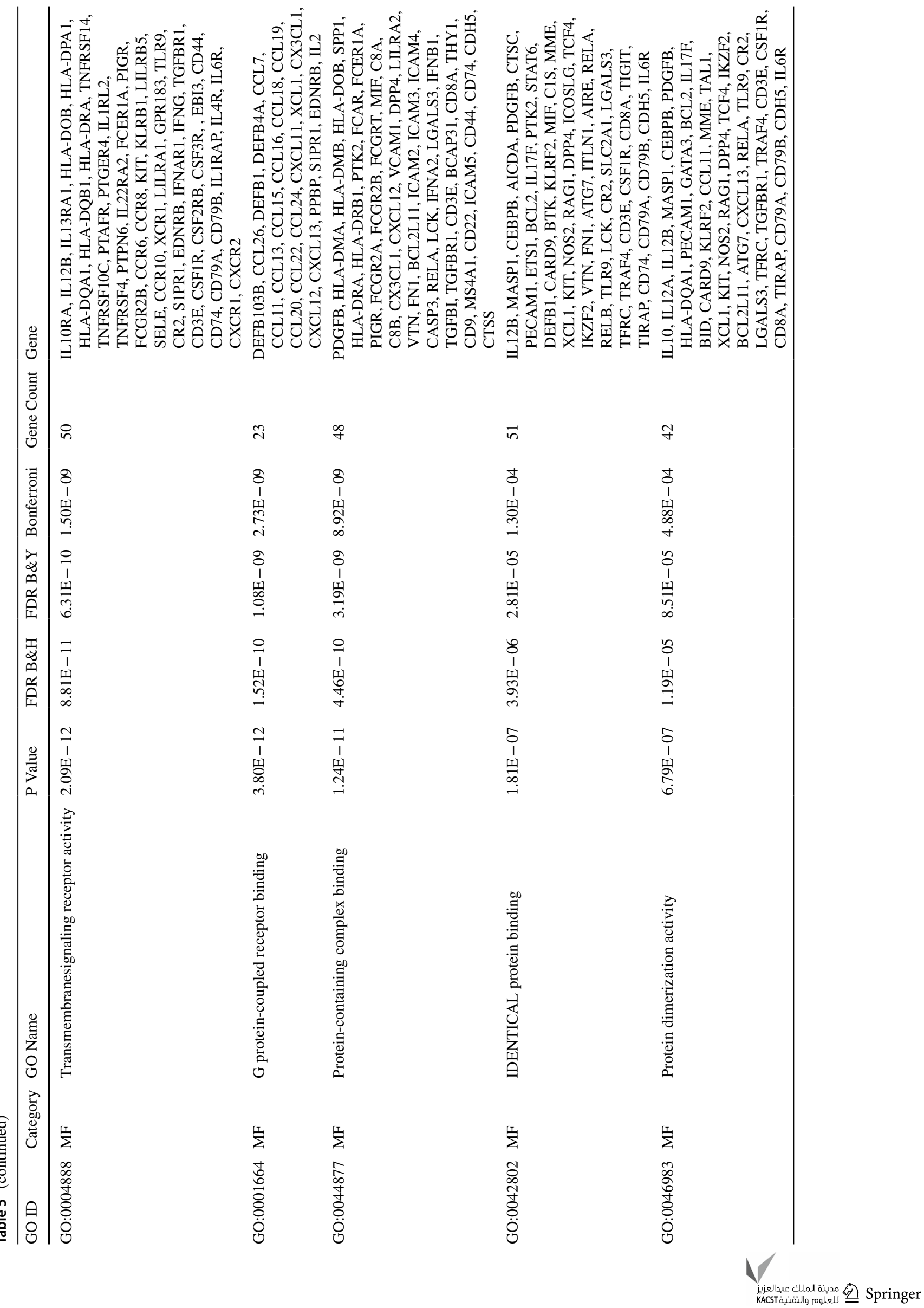


PEWCC1. The top four significant modules of up-regulated were selected for further analysis (Fig. 10). Module 48 had 10 nodes and 34 edges, module 50 had 10 nodes and 33 edges, Module 64 had 10 nodes and 17 edges and module 65 had 10 nodes and 17 edges, respectively. Enrichment analysis showed that the genes in these modules were mainly involved in natural killer cell-mediated cytotoxicity, measles, Jak-STAT signaling pathway, viral myocarditis, herpes simplex infection, influenza A, osteoclast differentiation, HTLVI infection, IL12-mediated signaling events, IL2-mediated signaling events, tuberculosis, malaria, paxillin-dependent events mediated by a4b1, TCR signaling in naive CD $8+T$ cells and cytokine signaling in Immune system. The top four significant modules of down-regulated were selected for further analysis (Fig. 11). Module 18 had 17 nodes and 44 edges, module 23 had 15 nodes and 20 edges, module 58 had 9 nodes and 22 edges and module 104 had 7 nodes and 12 edges, respectively. Enrichment analysis showed that the genes in these modules were mainly involved in apoptosis signaling pathway, tuberculosis, viral myocarditis, Jak-STAT signaling pathway, cytokine signaling in immune system, measles, innate immune system, cytokine-cytokine receptor interaction, influenza A, hematopoietic cell lineage, HTLV-I infection, signaling by interleukins, adaptive immune system, IL12-mediated signaling events, interleukin signaling pathway, inflammation mediated by chemokine and cytokine signaling pathway, inflammatory bowel disease (IBD), FAS (CD95) signaling pathway, cytokine-mediated signaling pathway, response to biotic stimulus, IL4-mediated signaling events, MAPK family signaling cascades and regulation of immune system process.

\section{Construction of target gene-miRNA regulatory network}

Using the miRNet database, target gene-miRNA regulatory network for up-regulated genes had 1008 nodes and 1613 interactions (Fig. 12). The network marked that each target genes have interactions with miRNAs. IKZF3 regulates 134 miRNAs (ex, hsa-mir-6860), TP53 regulates 130 miRNAs (ex, hsa-mir-5703), IFNAR2 regulates 109 miRNAs (ex, hsa-mir - 4510), SMAD5 regulates 83 miRNAs (ex, hsa-mir-6086) and STAT3 regulates by 80 miRNAs (ex, hsa-mir -4270) are listed in Table 8. Enrichment analysis showed that the target genes in this network were mainly involved in IL2-mediated signaling events, measles, herpes simplex infection, ALK2 signaling events and cytokine signaling in immune system. Similarly, target gene-miRNA regulatory network for down-regulated genes had 1791 nodes and 3951 interactions (Fig. 13). SKI regulates 210 miRNAs (ex, hsa-mir-5100), TNFRSF13C regulates 136 miRNAs (ex, hsa-mir-3197), BCL2L11 regulates 122 miRNAs (ex, hsa-mir-8064), ICOSLG regulates 119 miRNAs 


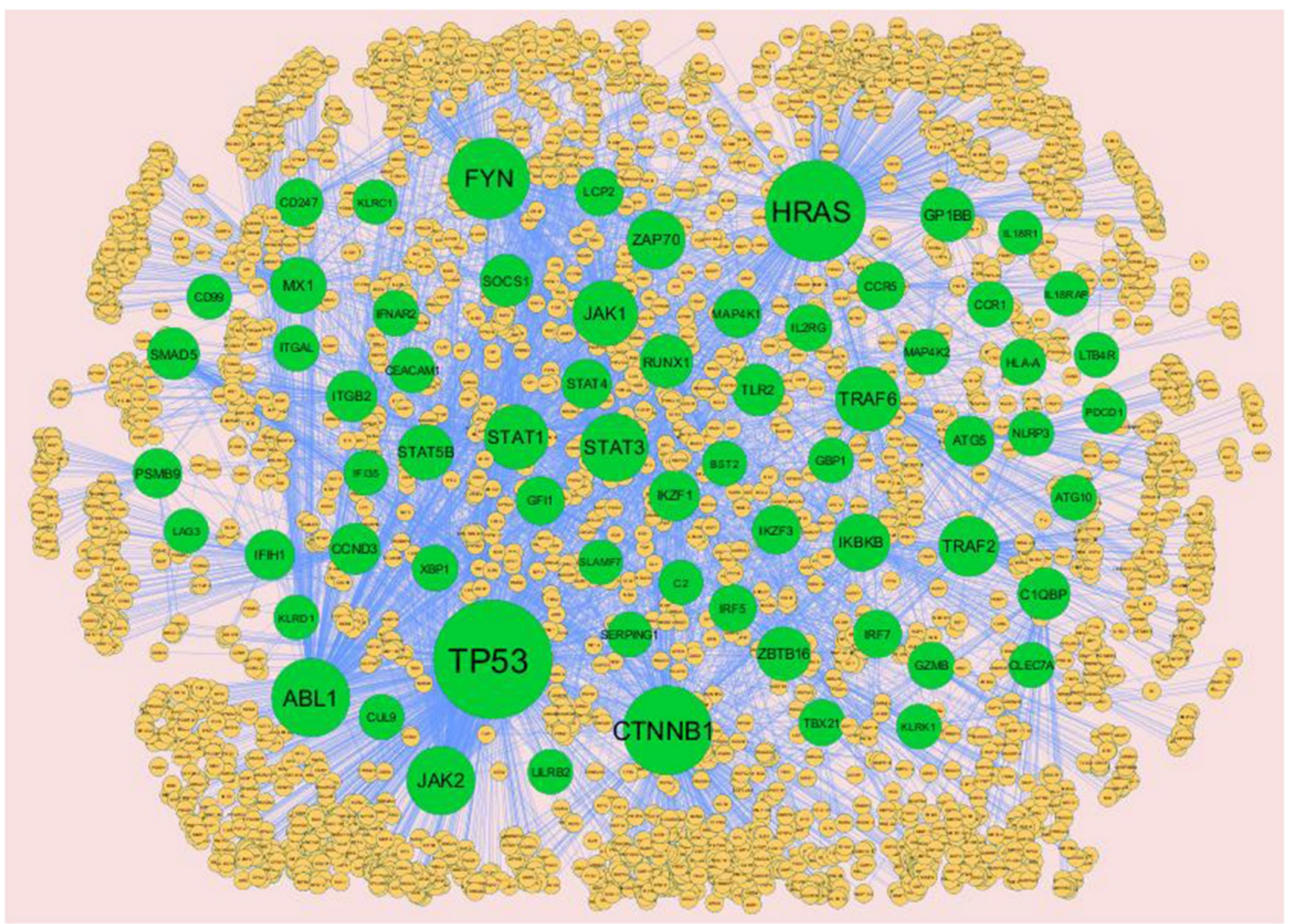

Fig. 6 Protein-protein interaction network of up regulated genes. Green nodes ( -$)$ denotes up regulated genes; Blue lines ( - ) denotes edges (Interactions)

(ex, hsa-mir-3672) and IL6R regulates 94 miRNAs (ex, hsamir-7641) are listed in Table 7. Enrichment analysis showed that the target genes in this network were mainly involved in molecular function regulator, cytokine-cytokine receptor interaction, apoptosis signaling pathway, adaptive immune system and cytokine-cytokine receptor interaction.

\section{Construction of target gene-TF regulatory network}

Using the NetworkAnalyst database, target gene-TF regulatory network for up-regulated genes had 145 nodes and 634 interactions (Fig. 14). The network marked that each target genes have interactions with transcription factors (TFs). JAK1 regulates 46 TFs (ex, FOXC1), TRAF6 regulates $31 \mathrm{TFs}$ (ex, GATA2), CLEC7A regulates 25 TFs (ex, YY1), STAT1 regulates 22 TFs (ex, CREB1) and IKZF1 regulates 22 TFs (ex, TFAP2A) are listed in Table 8. Enrichment analysis showed that the target genes in this network were mainly involved in measles, herpes simplex infection, tuberculosis, osteoclast differentiation and regulation of immune system process. Similarly, target gene-TF regulatory network of down-regulated genes had 1788 nodes and 235 interactions (Fig. 15). KLRF2 regulates 127 TFs (ex, FOXC1), CD1A regulates 102 TFs (ex, GATA2), TNFRSF4 regulates 75 TFs (ex, YY1), MME regulates 63 TFs (ex, FOXL1) and CXCL12 regulates 63 TFs (ex, FOXL1) are listed in Table 7. Enrichment analysis showed that the target genes in this network were mainly involved in cytokine-mediated signaling pathway, hematopoietic cell lineage, cytokine-cytokine receptor interaction, innate immune system and peptide ligandbinding receptors.

\section{Validation of hub genes}

The ROC curve analysis was accomplished to assess the diagnostic values of hub genes. Our finding revealed that CCL5 $(\mathrm{AUC}=0.784)$, IFNAR2 $(\mathrm{AUC}=0.750)$, JAK2 $($ AUC $=0.859)$, MX1 $($ AUC $=0.773)$, STAT 1 
Table 6 Topology table for up and down regulated genes

\begin{tabular}{|c|c|c|c|c|c|c|}
\hline Regulation & Node & Degree & Betweenness & Stress & Closeness & Clustering Coefficient \\
\hline $\mathrm{Up}$ & TP53 & 682 & 0.308256 & 69419762 & 0.44613 & 0.001042 \\
\hline Up & HRAS & 543 & 0.243425 & 36147274 & 0.454702 & 0.003568 \\
\hline Up & CTNNB1 & 454 & 0.17049 & 60805652 & 0.403018 & 0.002042 \\
\hline Up & FYN & 396 & 0.117564 & 12570856 & 0.436236 & 0.010907 \\
\hline Up & ABL1 & 362 & 0.118179 & 7631538 & 0.470883 & 0.015228 \\
\hline Up & JAK2 & 258 & 0.054758 & 7164820 & 0.434802 & 0.021748 \\
\hline Up & STAT3 & 253 & 0.05294 & 16470742 & 0.398767 & 0.02108 \\
\hline Up & JAK1 & 223 & 0.032981 & 6336566 & 0.40235 & 0.019876 \\
\hline Up & TRAF6 & 216 & 0.074546 & 12176648 & 0.395301 & 0.010207 \\
\hline Up & STAT1 & 213 & 0.045072 & 12845532 & 0.387307 & 0.023031 \\
\hline Up & TRAF2 & 171 & 0.047572 & 11238720 & 0.357837 & 0.007981 \\
\hline Up & ZAP70 & 153 & 0.018814 & 3346658 & 0.375226 & 0.028294 \\
\hline Up & IKBKB & 140 & 0.040639 & 5153454 & 0.403521 & 0.017575 \\
\hline Up & MX1 & 125 & 0.057134 & 6475242 & 0.352549 & 0.002194 \\
\hline Up & STAT5B & 118 & 0.012758 & 2934924 & 0.377415 & 0.057801 \\
\hline Up & ZВТВ16 & 105 & 0.029011 & 4339392 & 0.371064 & 0.00696 \\
\hline Up & GP1BB & 104 & 0.024662 & 2308816 & 0.350596 & 0.009335 \\
\hline Up & RUNX1 & 95 & 0.021204 & 3579930 & 0.370309 & 0.010526 \\
\hline Up & C1QBP & 89 & 0.043687 & 7011558 & 0.334675 & 0 \\
\hline Up & TLR2 & 85 & 0.019615 & 2401022 & 0.374839 & 0.034734 \\
\hline Up & SOCS1 & 85 & 0.019595 & 1846156 & 0.398713 & 0.072829 \\
\hline Up & SMAD5 & 82 & 0.024208 & 4462604 & 0.336687 & 0.003011 \\
\hline Up & ITGB2 & 72 & 0.025976 & 3349086 & 0.351061 & 0.008607 \\
\hline Up & IKZF1 & 72 & 0.011714 & 1722502 & 0.373157 & 0.034038 \\
\hline Up & CCND3 & 71 & 0.025956 & 2361424 & 0.357661 & 0.008048 \\
\hline Up & ATG5 & 60 & 0.020131 & 2294688 & 0.333219 & 0.00678 \\
\hline Up & IFIH1 & 59 & 0.023459 & 2059722 & 0.333945 & 0.001753 \\
\hline Up & STAT4 & 58 & 0.002561 & 797810 & 0.366902 & 0.082275 \\
\hline Up & PSMB9 & 55 & 0.0248 & 2740768 & 0.310739 & 0 \\
\hline Up & IKZF3 & 47 & 0.005026 & 756292 & 0.361346 & 0.053654 \\
\hline Up & GFI1 & 46 & 0.007563 & 811180 & 0.357266 & 0.013527 \\
\hline Up & IRF7 & 44 & 0.010411 & 1198488 & 0.338804 & 0.008457 \\
\hline Up & MAP4K1 & 44 & 0.006432 & 851692 & 0.348957 & 0.046512 \\
\hline Up & CD247 & 43 & 0.00724 & 710792 & 0.349292 & 0.058693 \\
\hline Up & LCP2 & 41 & 0.003353 & 442356 & 0.338331 & 0.07439 \\
\hline Up & CCR5 & 39 & 0.010719 & 942222 & 0.343359 & 0.043185 \\
\hline Up & IRF5 & 34 & 0.006222 & 736764 & 0.339277 & 0.012478 \\
\hline Up & GZMB & 29 & 0.01009 & 1297184 & 0.313584 & 0 \\
\hline Up & IL2RG & 27 & 0.005284 & 347620 & 0.3463 & 0.071225 \\
\hline Up & TBX21 & 23 & 0.003781 & 493896 & 0.328704 & 0.023715 \\
\hline $\mathrm{Up}$ & XBP1 & 21 & 0.004344 & 440004 & 0.329933 & 0 \\
\hline Up & HLA-A & 19 & 0.007447 & 822622 & 0.311637 & 0 \\
\hline Up & MAP4K2 & 18 & 0.002038 & 190802 & 0.334636 & 0.045752 \\
\hline Up & CCR1 & 18 & 0.004542 & 722956 & 0.323912 & 0.019608 \\
\hline Up & IFNAR2 & 17 & 0.003686 & 214358 & 0.335872 & 0.169118 \\
\hline Up & ITGAL & 17 & 0.004258 & 302784 & 0.285588 & 0.058824 \\
\hline Up & ATG10 & 16 & 0.00303 & 204222 & 0.278618 & 0.075 \\
\hline Up & CEACAM1 & 15 & 0.003567 & 815206 & 0.308991 & 0 \\
\hline Up & CLEC7A & 13 & 0.006223 & 422114 & 0.302253 & 0 \\
\hline Up & CUL9 & 10 & 0.001917 & 66468 & 0.352806 & 0.111111 \\
\hline $\mathrm{Up}$ & SERPING1 & 10 & 0.004857 & 805696 & 0.203225 & 0 \\
\hline
\end{tabular}


Table 6 (continued)

\begin{tabular}{|c|c|c|c|c|c|c|}
\hline Regulation & Node & Degree & Betweenness & Stress & Closeness & Clustering Coefficient \\
\hline Up & NLRP3 & 10 & 0.002325 & 208526 & 0.304371 & 0 \\
\hline Up & LTB4R & 9 & 0.002812 & 970594 & 0.242059 & 0 \\
\hline Up & GBP1 & 9 & 0.001571 & 66852 & 0.307489 & 0 \\
\hline Up & LILRB2 & 9 & 0.004803 & 191694 & 0.287677 & 0 \\
\hline Up & CD99 & 8 & 0.002792 & 99752 & 0.324129 & 0 \\
\hline Up & KLRK1 & 7 & 0.003445 & 544176 & 0.26256 & 0 \\
\hline Up & BST2 & 7 & $1.93 \mathrm{E}-04$ & 36836 & 0.324273 & 0 \\
\hline Up & KLRD1 & 7 & 0.001389 & 97132 & 0.22517 & 0.047619 \\
\hline Up & KLRC1 & 5 & 0.003221 & 307724 & 0.286234 & 0.1 \\
\hline Up & IL18R1 & 4 & 0.00118 & 348338 & 0.245924 & 0 \\
\hline Up & PDCD1 & 4 & 0.001374 & 55018 & 0.303894 & 0.166667 \\
\hline Up & SLAMF7 & 4 & 0.00206 & 191070 & 0.233272 & 0 \\
\hline Up & $\mathrm{C} 2$ & 4 & 0.001446 & 96444 & 0.245447 & 0 \\
\hline Up & IFI35 & 3 & $6.93 E-04$ & 23710 & 0.323301 & 0 \\
\hline Up & LAG3 & 2 & $4.23 \mathrm{E}-06$ & 1074 & 0.245695 & 0 \\
\hline Up & IL18RAP & 2 & $1.95 \mathrm{E}-04$ & 52194 & 0.233609 & 0 \\
\hline Down & MAPK11 & 421 & 0.179413 & 26118522 & 0.388195 & 0.003744 \\
\hline Down & RELA & 285 & 0.126824 & 20931198 & 0.37804 & 0.004645 \\
\hline Down & LCK & 267 & 0.108492 & 11305268 & 0.405534 & 0.014108 \\
\hline Down & KIT & 221 & 0.051071 & 10158794 & 0.36783 & 0.015014 \\
\hline Down & EGR1 & 195 & 0.060886 & 6334914 & 0.384414 & 0.016178 \\
\hline Down & ILF3 & 179 & 0.093546 & 7805892 & 0.330116 & $3.14 \mathrm{E}-04$ \\
\hline Down & CASP3 & 175 & 0.070326 & 10842834 & 0.355181 & 0.005123 \\
\hline Down & PTK2 & 153 & 0.049652 & 8738870 & 0.356704 & 0.005246 \\
\hline Down & PTPN6 & 150 & 0.044684 & 5323296 & 0.365645 & 0.021477 \\
\hline Down & BCL2 & 150 & 0.062256 & 5772430 & 0.365862 & 0.009128 \\
\hline Down & BTK & 137 & 0.022999 & 3449618 & 0.35359 & 0.019429 \\
\hline Down & STAT5A & 126 & 0.024378 & 5096524 & 0.35745 & 0.027429 \\
\hline Down & TGFBR1 & 123 & 0.048955 & 6050368 & 0.349257 & 0.007464 \\
\hline Down & CSF1R & 117 & 0.01327 & 3411424 & 0.344835 & 0.005894 \\
\hline Down & FN1 & 116 & 0.052141 & 5201654 & 0.335331 & 0.003148 \\
\hline Down & TCF4 & 115 & 0.044569 & 5794596 & 0.333586 & 0.005492 \\
\hline Down & TRAF3 & 113 & 0.038869 & 4088284 & 0.341886 & 0.010588 \\
\hline Down & STAT6 & 102 & 0.026319 & 3212830 & 0.363486 & 0.039216 \\
\hline Down & GATA3 & 97 & 0.03064 & 3006348 & 0.350689 & 0.018471 \\
\hline Down & IRAK4 & 90 & 0.015953 & 2526624 & 0.344064 & 0.010986 \\
\hline Down & TCF7 & 90 & 0.029254 & 4190222 & 0.323796 & $9.99 \mathrm{E}-04$ \\
\hline Down & ATG7 & 88 & 0.032475 & 4557368 & 0.323761 & 0 \\
\hline Down & PSMB10 & 86 & 0.037084 & 3948650 & 0.316536 & 0 \\
\hline Down & EGR2 & 78 & 0.014472 & 2942228 & 0.323966 & 0.002331 \\
\hline Down & CEBPB & 77 & 0.01563 & 2080316 & 0.347208 & 0.028708 \\
\hline Down & TLR9 & 75 & 0.01613 & 2504516 & 0.335623 & 0.017658 \\
\hline Down & ETS1 & 74 & 0.015119 & 2229504 & 0.345493 & 0.023695 \\
\hline Down & MBP & 69 & 0.02034 & 1946892 & 0.33211 & 0.008099 \\
\hline Down & CD40 & 63 & 0.012367 & 1565636 & 0.343144 & 0.030722 \\
\hline Down & PTPN22 & 63 & 0.005103 & 648768 & 0.333009 & 0.039939 \\
\hline Down & CXCL13 & 62 & 0.033409 & 4616616 & 0.255924 & 0.002644 \\
\hline Down & CD44 & 61 & 0.027234 & 2621678 & 0.352941 & 0.016393 \\
\hline Down & DUSP4 & 56 & 0.005964 & 877004 & 0.343106 & 0.046753 \\
\hline Down & TOLLIP & 55 & 0.020841 & 1681614 & 0.32948 & 0.011448 \\
\hline Down & TRAF4 & 54 & 0.017367 & 1322968 & 0.327447 & 0.012579 \\
\hline
\end{tabular}


Table 6 (continued)

\begin{tabular}{|c|c|c|c|c|c|c|}
\hline Regulation & Node & Degree & Betweenness & Stress & Closeness & Clustering Coefficient \\
\hline Down & TFRC & 51 & 0.020865 & 2030850 & 0.323932 & $7.84 \mathrm{E}-04$ \\
\hline Down & TAL1 & 49 & 0.012074 & 1294452 & 0.327273 & 0.02551 \\
\hline Down & BATF3 & 48 & 0.009925 & 1237560 & 0.309471 & 0.011525 \\
\hline Down & SKI & 47 & 0.009912 & 1441494 & 0.325026 & 0.010176 \\
\hline Down & MAP4K4 & 46 & 0.00723 & 923942 & 0.334966 & 0.025121 \\
\hline Down & ARHGDIB & 43 & 0.008007 & 998356 & 0.325267 & 0.00443 \\
\hline Down & BID & 40 & 0.007734 & 665116 & 0.336026 & 0.046154 \\
\hline Down & BCL2L11 & 39 & 0.006742 & 718322 & 0.327935 & 0.032389 \\
\hline Down & NOS2 & 39 & 0.012743 & 915032 & 0.332218 & 0.010796 \\
\hline Down & AICDA & 38 & 0.016444 & 2864544 & 0.288094 & 0 \\
\hline Down & GPI & 37 & 0.009895 & 1400722 & 0.322202 & 0 \\
\hline Down & CSF2RB & 37 & 0.005071 & 681430 & 0.345571 & 0.100601 \\
\hline Down & TIRAP & 37 & 0.009595 & 1070880 & 0.324 & 0.033033 \\
\hline Down & CD9 & 36 & 0.01001 & 838100 & 0.302685 & 0.019048 \\
\hline Down & $\mathrm{CDH} 5$ & 35 & 0.00845 & 737334 & 0.306177 & 0.013445 \\
\hline Down & AIRE & 35 & 0.010893 & 2140272 & 0.292753 & 0 \\
\hline Down & CD82 & 32 & 0.008605 & 911074 & 0.323659 & 0.020161 \\
\hline Down & IFNAR1 & 31 & 0.006943 & 536824 & 0.340902 & 0.062366 \\
\hline Down & CD3E & 30 & 0.006779 & 602042 & 0.338614 & 0.078161 \\
\hline Down & LGALS3 & 30 & 0.009645 & 633882 & 0.302536 & 0 \\
\hline Down & VTN & 30 & 0.009755 & 750034 & 0.299445 & 0.006897 \\
\hline Down & CD8A & 29 & 0.008368 & 709958 & 0.334638 & 0.029557 \\
\hline Down & CSF3R & 28 & 0.008229 & 711320 & 0.334093 & 0.029101 \\
\hline Down & EOMES & 28 & 0.005201 & 513270 & 0.277597 & 0.018519 \\
\hline Down & VCAM1 & 28 & 0.008841 & 718322 & 0.31732 & 0.010582 \\
\hline Down & ZEB1 & 27 & 0.006122 & 1257304 & 0.288851 & 0 \\
\hline Down & MME & 27 & 0.009061 & 1835908 & 0.290624 & 0.014245 \\
\hline Down & MIF & 27 & 0.00826 & 611016 & 0.321596 & 0.005698 \\
\hline Down & IKZF2 & 26 & 0.003358 & 390862 & 0.3129 & 0 \\
\hline Down & MAPKAPK2 & 26 & 0.004801 & 451358 & 0.310909 & 0.024615 \\
\hline Down & PLAU & 25 & 0.008975 & 593824 & 0.317844 & 0.01 \\
\hline Down & DPP4 & 25 & 0.010538 & 1418468 & 0.265231 & 0.006667 \\
\hline Down & RORC & 25 & 0.00722 & 1661398 & 0.278729 & 0.013333 \\
\hline Down & CD55 & 24 & 0.010279 & 705586 & 0.303102 & 0 \\
\hline Down & IL4R & 24 & 0.00238 & 270352 & 0.331181 & 0.083333 \\
\hline Down & CD19 & 23 & 0.001702 & 211728 & 0.304301 & 0.090909 \\
\hline Down & IRF8 & 23 & 0.005195 & 372574 & 0.329904 & 0.019763 \\
\hline Down & IL4 & 23 & 0.004905 & 441962 & 0.296246 & 0.019763 \\
\hline Down & RELB & 23 & 0.003464 & 286340 & 0.326336 & 0.055336 \\
\hline Down & PAX5 & 22 & 0.002896 & 300204 & 0.291726 & 0.021645 \\
\hline Down & CXCR2 & 22 & 0.006344 & 1097670 & 0.26098 & 0.047619 \\
\hline Down & IL16 & 22 & 0.008141 & 455702 & 0.300821 & 0 \\
\hline Down & SPP1 & 21 & 0.005095 & 320668 & 0.307124 & 0.014286 \\
\hline Down & THY1 & 21 & 0.008614 & 468640 & 0.309285 & 0.004762 \\
\hline Down & SLC2A1 & 20 & 0.00547 & 544898 & 0.316244 & 0 \\
\hline Down & EDNRB & 20 & 0.006473 & 488214 & 0.313729 & 0 \\
\hline Down & SELE & 20 & 0.005852 & 411150 & 0.300615 & 0.031579 \\
\hline Down & HLA-DRA & 18 & 0.004735 & 398954 & 0.319992 & 0.052288 \\
\hline Down & CD79A & 18 & 0.00478 & 297512 & 0.338726 & 0.176471 \\
\hline Down & HLA-B & 17 & 0.006176 & 428434 & 0.315111 & 0.022059 \\
\hline Down & BCAP31 & 17 & 0.005172 & 568664 & 0.289476 & 0.058824 \\
\hline
\end{tabular}


Table 6 (continued)

\begin{tabular}{|c|c|c|c|c|c|c|}
\hline Regulation & Node & Degree & Betweenness & Stress & Closeness & Clustering Coefficient \\
\hline Down & IL1RAP & 17 & 0.003059 & 257852 & 0.322101 & 0.088235 \\
\hline Down & PDGFB & 16 & 0.003308 & 533330 & 0.27399 & 0 \\
\hline Down & S1PR1 & 16 & 0.002256 & 404754 & 0.320025 & 0 \\
\hline Down & CD46 & 15 & 0.004006 & 360234 & 0.31814 & 0.047619 \\
\hline Down & RAG1 & 15 & 0.002698 & 229148 & 0.267304 & 0.019048 \\
\hline Down & $\mathrm{CD} 22$ & 15 & 0.003395 & 414458 & 0.289123 & 0.07619 \\
\hline Down & POU2F2 & 14 & 0.00252 & 256428 & 0.313506 & 0 \\
\hline Down & TNFRSF14 & 14 & 0.002863 & 205148 & 0.290131 & 0.054945 \\
\hline Down & FCGR2B & 14 & 0.001419 & 233096 & 0.299854 & 0.087912 \\
\hline Down & CSF2 & 14 & 0.001964 & 189924 & 0.271764 & 0.076923 \\
\hline Down & SELPLG & 14 & 0.004641 & 618406 & 0.266933 & 0.010989 \\
\hline Down & IL10 & 13 & 0.001286 & 131502 & 0.275387 & 0.025641 \\
\hline Down & CCL7 & 13 & 0.002891 & 346838 & 0.240789 & 0 \\
\hline Down & CD79B & 13 & $3.06 \mathrm{E}-04$ & 78608 & 0.337796 & 0.423077 \\
\hline Down & HLA-C & 13 & 0.003625 & 407006 & 0.310407 & 0.025641 \\
\hline Down & IL6R & 13 & 0.004275 & 289876 & 0.288689 & 0.051282 \\
\hline Down & IL17A & 13 & 0.002334 & 388710 & 0.280328 & 0.025641 \\
\hline Down & FCGR2A & 13 & 0.002143 & 252800 & 0.318403 & 0 \\
\hline Down & LTA & 12 & 0.002886 & 476206 & 0.257315 & 0 \\
\hline Down & CXCL12 & 12 & 0.005496 & 372448 & 0.27655 & 0.030303 \\
\hline Down & CTSS & 12 & 0.00407 & 451030 & 0.260472 & 0 \\
\hline Down & CD59 & 12 & 0.004363 & 207460 & 0.290405 & 0.030303 \\
\hline Down & CXCR1 & 12 & $8.07 \mathrm{E}-04$ & 176404 & 0.242095 & 0.166667 \\
\hline Down & IL2 & 12 & 0.002493 & 217616 & 0.290487 & 0.030303 \\
\hline Down & C6 & 12 & $9.26 \mathrm{E}-04$ & 133246 & 0.24911 & 0 \\
\hline Down & CD74 & 12 & 0.003192 & 267306 & 0.315369 & 0.121212 \\
\hline Down & CR2 & 11 & 0.001319 & 143504 & 0.267072 & 0.036364 \\
\hline Down & CCR10 & 11 & 0.003702 & 303034 & 0.222963 & 0.072727 \\
\hline Down & CTSC & 11 & 0.004925 & 257558 & 0.302268 & 0 \\
\hline Down & TGFBI & 10 & $7.77 \mathrm{E}-04$ & 51700 & 0.253167 & 0.155556 \\
\hline Down & CD209 & 10 & $4.47 \mathrm{E}-04$ & 37928 & 0.243628 & 0.044444 \\
\hline Down & NT5E & 10 & 0.003249 & 169846 & 0.314627 & 0.022222 \\
\hline Down & IL10RA & 10 & 0.001722 & 123836 & 0.32116 & 0.155556 \\
\hline Down & MBL2 & 9 & 0.002733 & 247190 & 0.248106 & 0.027778 \\
\hline Down & TNFRSF9 & 9 & 0.001065 & 77604 & 0.309005 & 0.305556 \\
\hline Down & HLA-DQA1 & 9 & $8.50 \mathrm{E}-04$ & 84902 & 0.306147 & 0.194444 \\
\hline Down & NFIL3 & 9 & 0.002148 & 269802 & 0.253063 & 0 \\
\hline Down & MS4A1 & 9 & $6.53 \mathrm{E}-04$ & 54246 & 0.308664 & 0.083333 \\
\hline Down & PTAFR & 9 & 0.001839 & 146258 & 0.315401 & 0.055556 \\
\hline Down & MASP1 & 9 & 0.002137 & 326806 & 0.225528 & 0.083333 \\
\hline Down & PLA2G2A & 8 & 0.002039 & 625578 & 0.228338 & 0 \\
\hline Down & TNFRSF4 & 8 & $7.20 \mathrm{E}-04$ & 87330 & 0.26198 & 0.321429 \\
\hline Down & IL13RA1 & 8 & $6.92 \mathrm{E}-04$ & 54632 & 0.3129 & 0.214286 \\
\hline Down & CD48 & 8 & 0.001004 & 75166 & 0.297881 & 0.142857 \\
\hline Down & CD244 & 8 & 0.001314 & 270208 & 0.265574 & 0.071429 \\
\hline Down & HLA-DRB1 & 8 & 0.001343 & 119046 & 0.273066 & 0.142857 \\
\hline Down & IL12A & 8 & 0.003272 & 296440 & 0.241412 & 0.071429 \\
\hline Down & ICAM3 & 8 & $6.74 \mathrm{E}-04$ & 94610 & 0.255118 & 0.035714 \\
\hline Down & ICAM2 & 7 & $7.74 \mathrm{E}-04$ & 115578 & 0.242687 & 0.047619 \\
\hline Down & CD86 & 7 & 0.00143 & 76566 & 0.302417 & 0.190476 \\
\hline Down & IL6 & 7 & 0.001988 & 115352 & 0.260582 & 0.095238 \\
\hline
\end{tabular}


Table 6 (continued)

\begin{tabular}{|c|c|c|c|c|c|c|}
\hline Regulation & Node & Degree & Betweenness & Stress & Closeness & Clustering Coefficient \\
\hline Down & CD80 & 7 & 0.001335 & 74000 & 0.254317 & 0.190476 \\
\hline Down & CCL13 & 7 & $3.79 \mathrm{E}-04$ & 117758 & 0.239347 & 0 \\
\hline Down & CARD9 & 6 & 0.001358 & 152030 & 0.262583 & 0 \\
\hline Down & MASP2 & 6 & $8.34 \mathrm{E}-04$ & 73116 & 0.210649 & 0.2 \\
\hline Down & HLA-DMA & 6 & 0.001965 & 186844 & 0.252792 & 0.2 \\
\hline Down & FCER1A & 6 & 0.001958 & 462984 & 0.26283 & 0 \\
\hline Down & IL13 & 6 & $9.18 \mathrm{E}-04$ & 81904 & 0.260164 & 0.2 \\
\hline Down & PIGR & 6 & 0.001383 & 46460 & 0.267211 & 0 \\
\hline Down & ICAM4 & 6 & $7.08 \mathrm{E}-04$ & 122662 & 0.244131 & 0 \\
\hline Down & CCL11 & 6 & $4.36 \mathrm{E}-04$ & 106892 & 0.239682 & 0 \\
\hline Down & FCAR & 6 & 0.001325 & 60556 & 0.281404 & 0.066667 \\
\hline Down & PTGER4 & 6 & 0.001594 & 111600 & 0.311981 & 0 \\
\hline Down & RAG2 & 6 & 0.002235 & 108180 & 0.302893 & 0.133333 \\
\hline Down & РPBP & 6 & 0.00149 & 101978 & 0.25057 & 0.066667 \\
\hline Down & IL3 & 6 & $1.42 \mathrm{E}-05$ & 4398 & 0.276848 & 0.133333 \\
\hline Down & HLA-DQB1 & 5 & $1.37 \mathrm{E}-04$ & 32052 & 0.304361 & 0.5 \\
\hline Down & IDO1 & 5 & 0.002598 & 124836 & 0.300263 & 0 \\
\hline Down & TNFRSF10C & 5 & $6.74 \mathrm{E}-04$ & 112660 & 0.252006 & 0 \\
\hline Down & TNFRSF13C & 5 & $6.50 \mathrm{E}-04$ & 58590 & 0.256308 & 0.3 \\
\hline Down & IL17F & 5 & $2.32 \mathrm{E}-04$ & 81308 & 0.256031 & 0.2 \\
\hline Down & CFD & 5 & 0.001535 & 135276 & 0.301765 & 0 \\
\hline Down & CCL19 & 5 & $8.76 \mathrm{E}-05$ & 9268 & 0.219387 & 0.4 \\
\hline Down & CD34 & 5 & 0.001329 & 226272 & 0.243224 & 0 \\
\hline Down & IFNG & 5 & 0.001405 & 110736 & 0.270285 & 0 \\
\hline Down & IL23A & 5 & 0.00132 & 310874 & 0.180846 & 0.1 \\
\hline Down & EBI3 & 5 & 0.001288 & 90012 & 0.222769 & 0.1 \\
\hline Down & $\mathrm{C} 8 \mathrm{~B}$ & 4 & $2.59 \mathrm{E}-04$ & 21386 & 0.208212 & 0.166667 \\
\hline Down & CCL20 & 4 & 0.001697 & 215266 & 0.242974 & 0 \\
\hline Down & C4BPA & 4 & 0.001305 & 202342 & 0.207217 & 0 \\
\hline Down & C8A & 4 & $5.17 \mathrm{E}-04$ & 27442 & 0.225528 & 0.333333 \\
\hline Down & SLAMF6 & 4 & $6.38 \mathrm{E}-06$ & 766 & 0.274209 & 0.333333 \\
\hline Down & CCR6 & 4 & 0.001956 & 273648 & 0.209231 & 0 \\
\hline Down & BTLA & 4 & $6.84 \mathrm{E}-04$ & 61880 & 0.275584 & 0.333333 \\
\hline Down & CCR8 & 4 & $6.64 \mathrm{E}-04$ & 48144 & 0.196954 & 0 \\
\hline Down & CXCL11 & 4 & $8.55 \mathrm{E}-05$ & 11782 & 0.220629 & 0 \\
\hline Down & SIGIRR & 4 & $3.08 \mathrm{E}-06$ & 666 & 0.277147 & 0.333333 \\
\hline Down & ITLN1 & 4 & 0.001313 & 85710 & 0.272679 & 0 \\
\hline Down & ICAM5 & 4 & $3.97 \mathrm{E}-05$ & 5052 & 0.242936 & 0 \\
\hline Down & FCGRT & 4 & 0.001307 & 236680 & 0.210779 & 0 \\
\hline Down & CCL22 & 4 & $5.04 \mathrm{E}-04$ & 43848 & 0.247169 & 0 \\
\hline Down & IL12B & 4 & $1.64 \mathrm{E}-04$ & 39054 & 0.194859 & 0.5 \\
\hline Down & IL27 & 3 & $2.87 \mathrm{E}-04$ & 26194 & 0.215697 & 0.333333 \\
\hline Down & IL22 & 3 & $6.54 \mathrm{E}-04$ & 37474 & 0.199417 & 0 \\
\hline Down & $\mathrm{C} 7$ & 3 & $1.54 \mathrm{E}-04$ & 29126 & 0.226657 & 0 \\
\hline Down & TIGIT & 3 & 0.001299 & 79710 & 0.18742 & 0 \\
\hline Down & DEFB4A & 3 & 0.002265 & 309432 & 0.250917 & 0 \\
\hline Down & IL22RA2 & 3 & $8.49 \mathrm{E}-04$ & 82918 & 0.216806 & 0 \\
\hline Down & IL21 & 3 & $2.06 \mathrm{E}-05$ & 882 & 0.21301 & 0 \\
\hline Down & C9 & 3 & $1.97 \mathrm{E}-05$ & 976 & 0.229359 & 0.333333 \\
\hline Down & LIF & 3 & $1.35 \mathrm{E}-05$ & 994 & 0.252689 & 0 \\
\hline Down & ITGAE & 3 & $6.54 \mathrm{E}-04$ & 122018 & 0.226607 & 0 \\
\hline
\end{tabular}


Table 6 (continued)

\begin{tabular}{llclrll}
\hline Regulation & Node & Degree & Betweenness & Stress & Closeness & Clustering Coefficient \\
\hline Down & IL5 & 3 & $2.60 \mathrm{E}-05$ & 1936 & 0.26283 & 0.333333 \\
Down & HLA-DPB1 & 2 & 0 & 0 & 0.240356 & 1 \\
Down & CFI & 2 & $6.50 \mathrm{E}-04$ & 65960 & 0.196527 & 0 \\
Down & IFNB1 & 2 & 0 & 0 & 0.254275 & 1 \\
Down & IFNA2 & 2 & 0 & 0 & 0.254275 & 1 \\
Down & XCL1 & 2 & 0.001299 & 97076 & 0.208706 & 0 \\
Down & CD1A & 2 & $6.50 \mathrm{E}-04$ & 79654 & 0.20475 & 0 \\
Down & XCR1 & 2 & $6.50 \mathrm{E}-04$ & 48540 & 0.172698 & 0 \\
Down & DEFB103B & 2 & $6.50 \mathrm{E}-04$ & 91060 & 0.173057 & 0 \\
Down & IL7 & 2 & $6.20 \mathrm{E}-07$ & 68 & 0.219497 & 0 \\
Down & KLRB1 & 2 & $6.50 \mathrm{E}-04$ & 58078 & 0.267815 & 0 \\
Down & IL20 & 2 & 0.666667 & 4 & 0.75 & 0 \\
Down & HLA-DMB & 1 & 0 & 0 & 0.201796 & 0 \\
Down & HLA-DOB & 1 & 0 & 0 & 0.16794 & 0 \\
Down & ICOSLG & 1 & 0 & 0 & 0.200469 & 0 \\
Down & CD83 & 1 & 0 & 0 & 0.300029 & 0 \\
Down & GPR183 & 1 & 0 & 0 & 0.300029 & 0 \\
Down & IKBKAP & 1 & 0 & 0 & 0.250938 & 0 \\
Down & DEFB1 & 1 & 0 & 0 & 0.173038 & 0 \\
Down & IL1RL2 & 1 & 0 & 0 & 0.20772 & 0 \\
Down & IL19 & 1 & 0 & 0 & 0.5 & 0 \\
\hline & & & & & & \\
\hline
\end{tabular}

A

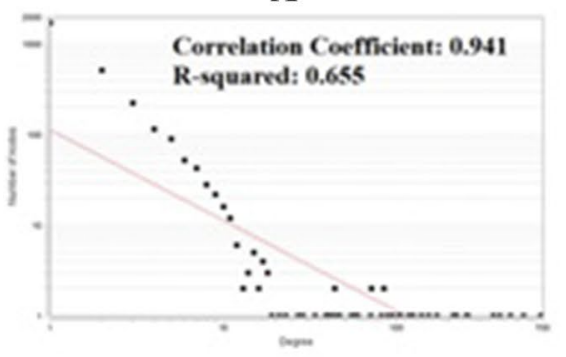

D

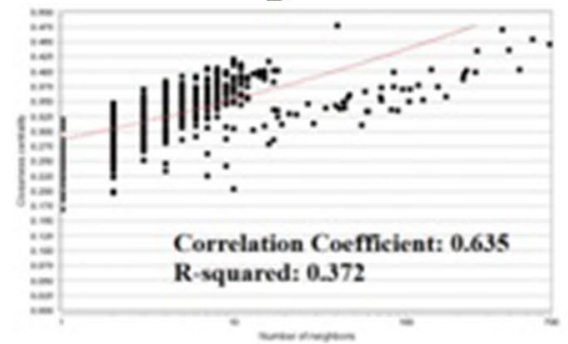

B

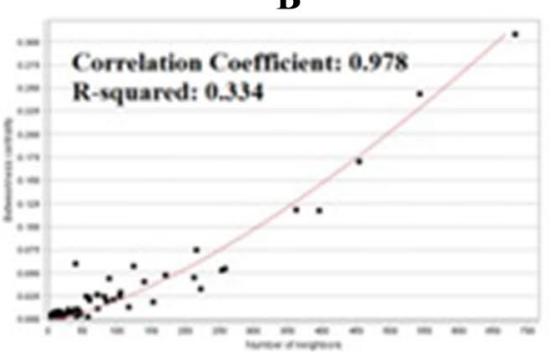

$\mathbf{E}$

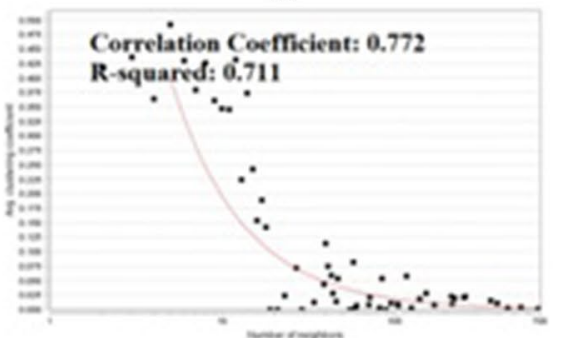

C

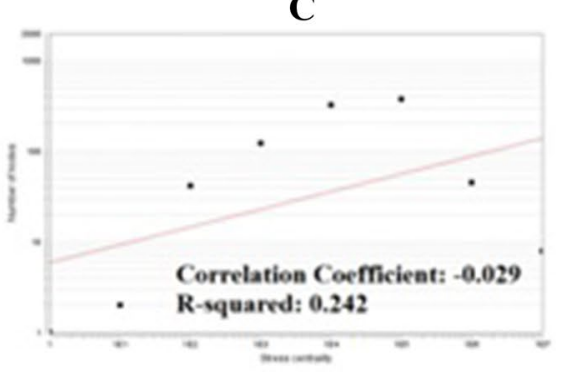

Correlation Coefricient: $-0,029$

Fig. 7 Scatter plot for up regulated genes. (A—Node degree; B-Betweenness centrality; C—Stress centrality; D—Closeness centrality; EClustering coefficient)

$(\mathrm{AUC}=0.873), \mathrm{BID}(\mathrm{AUC}=0.848), \mathrm{CD} 55(\mathrm{AUC}=0.973)$, CD80 (AUC $=0.870)$, HAL-B $(\mathrm{AUC}=0.816)$ and HLADMA $(A U C=0.730)$ had significant diagnostic values for discriminating SARS-CoV-2 samples and normal controls (Fig. 16).

\section{Discussion}

Currently, genetic and genomic-related exploration progress speedily and provide new prospect to illuminate the molecular pathogenesis of SARS-CoV-2 infections. And 


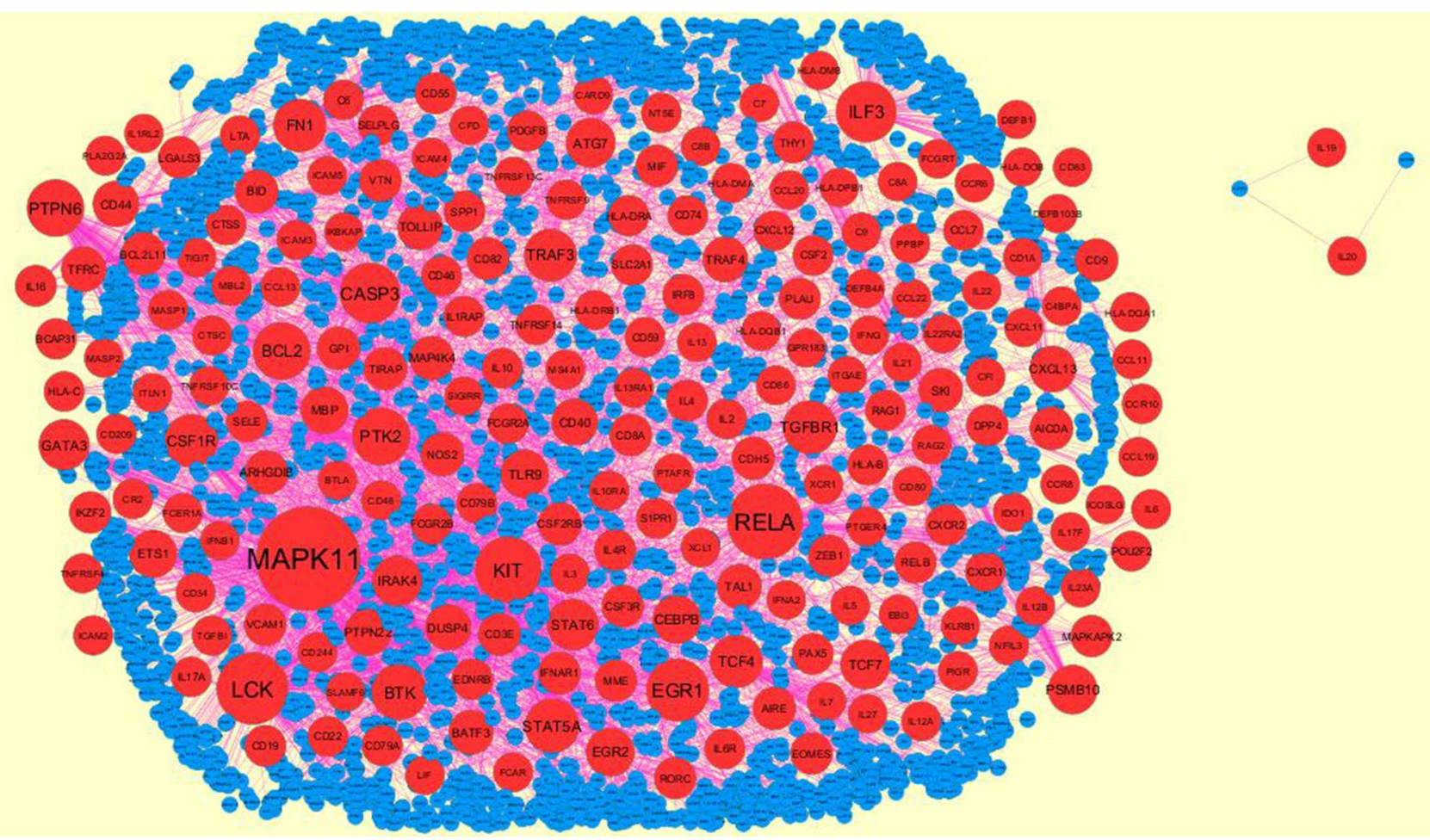

Fig. 8 Protein-protein interaction network of down regulated genes. Red nodes (-) denotes down regulated genes; Pink lines (-) denotes edges (Interactions)

A

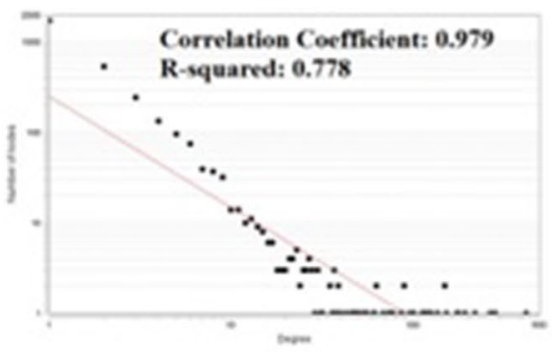

D

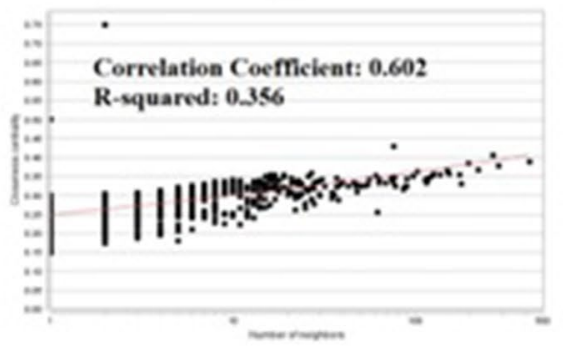

B

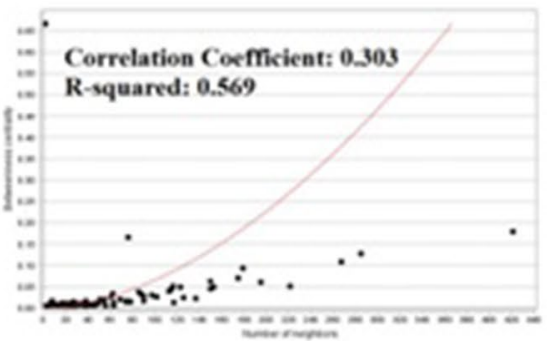

$\mathbf{E}$

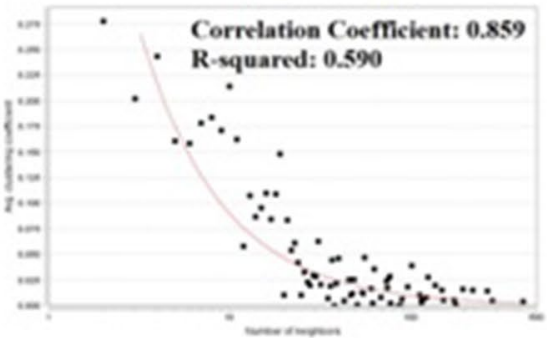

C

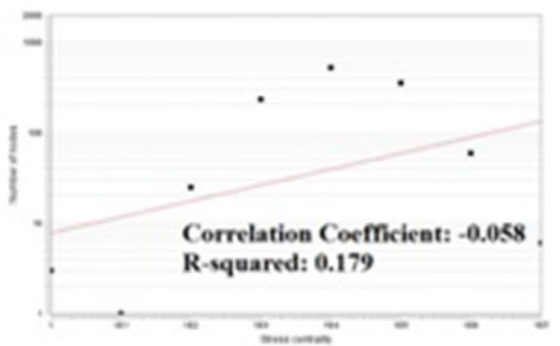

Correlation Coefficient: -0.058 ,

Fig. 9 Scatter plot for down regulated genes. (A-Node degree; B-Betweenness centrality; C-Stress centrality; D—Closeness centrality; EClustering coefficient)

bioinformatics analysis also has developed phenomenally and is committed to search for candidate biomarkers to implement more correct screening, prompt diagnosis for
SARS-CoV-2-infected patients based on enormous genetic and genomics data. 
Module 48

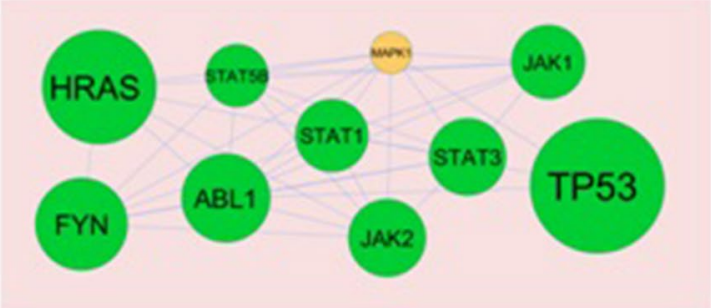

Module 64

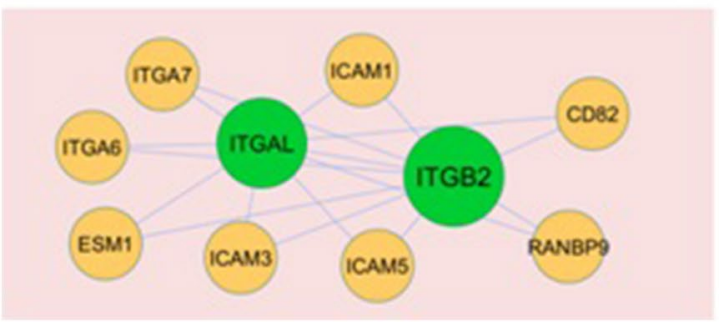

Module 50

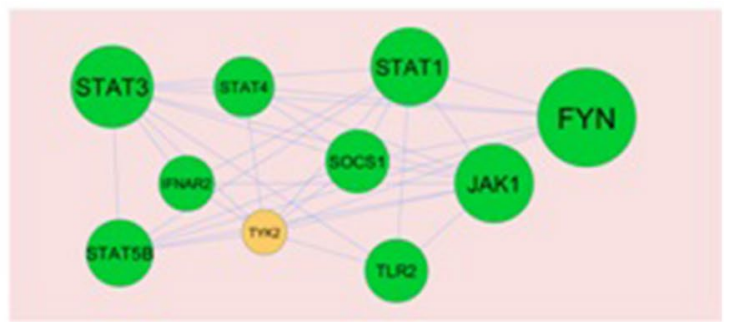

Module 65

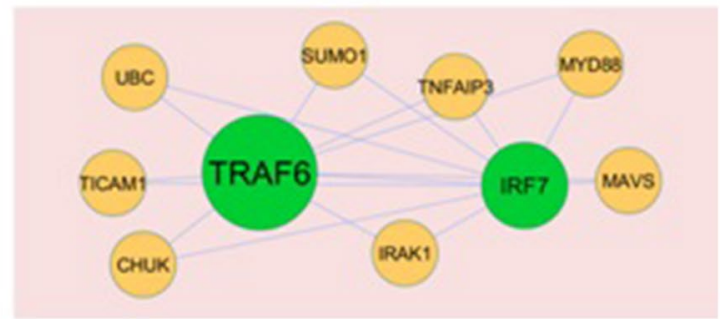

Fig. 10 Modules in PPI network. The green nodes denote the up regulated genes. Green nodes (-) denotes up regulated genes; Blue lines (-) denotes edges (Interactions)

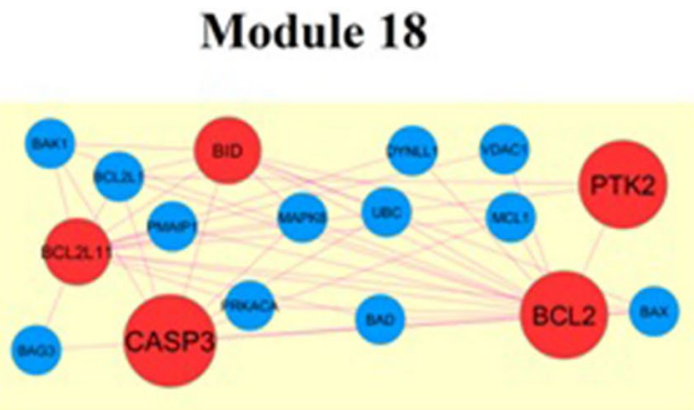

Module 58

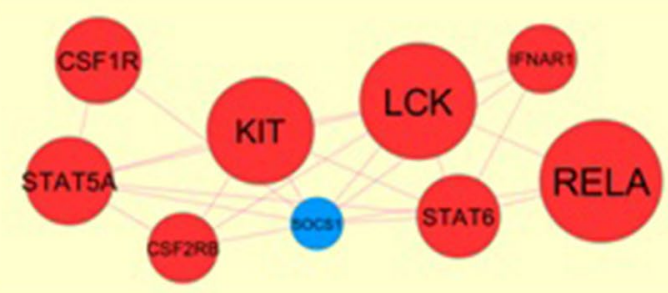

Module 23

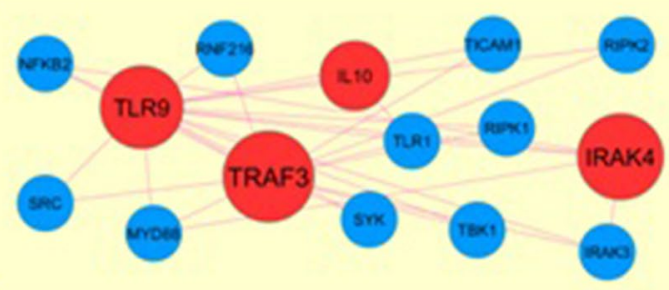

Module 104

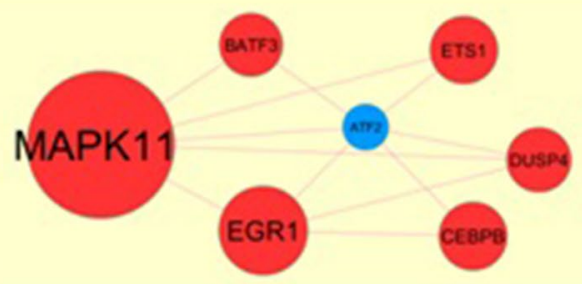

Fig. 11 Modules in PPI network. The red nodes denote the down regulated genes. Red nodes (f) denotes down regulated genes; Pink lines ( $(-)$ denotes edges (Interactions)

In the current investigation, a bioinformatics approach was used to identify candidate biomarker and therapeutic targets of SARS-CoV-2 infection. Following the analysis, 324 DEGs, including 76 up-regulated genes and 248 down-regulated genes were identified. Shi et al. (2007) found that expression of JAK1 was responsible for progression of adenovirus infection, but this gene may be linked with advancement of SARS-CoV-2 infection. Previously reported genes such as ZAP70 (Guntermann et al. 1997), CD22 (Ma et al. 2013) and MAPKAPK2 (Yang 


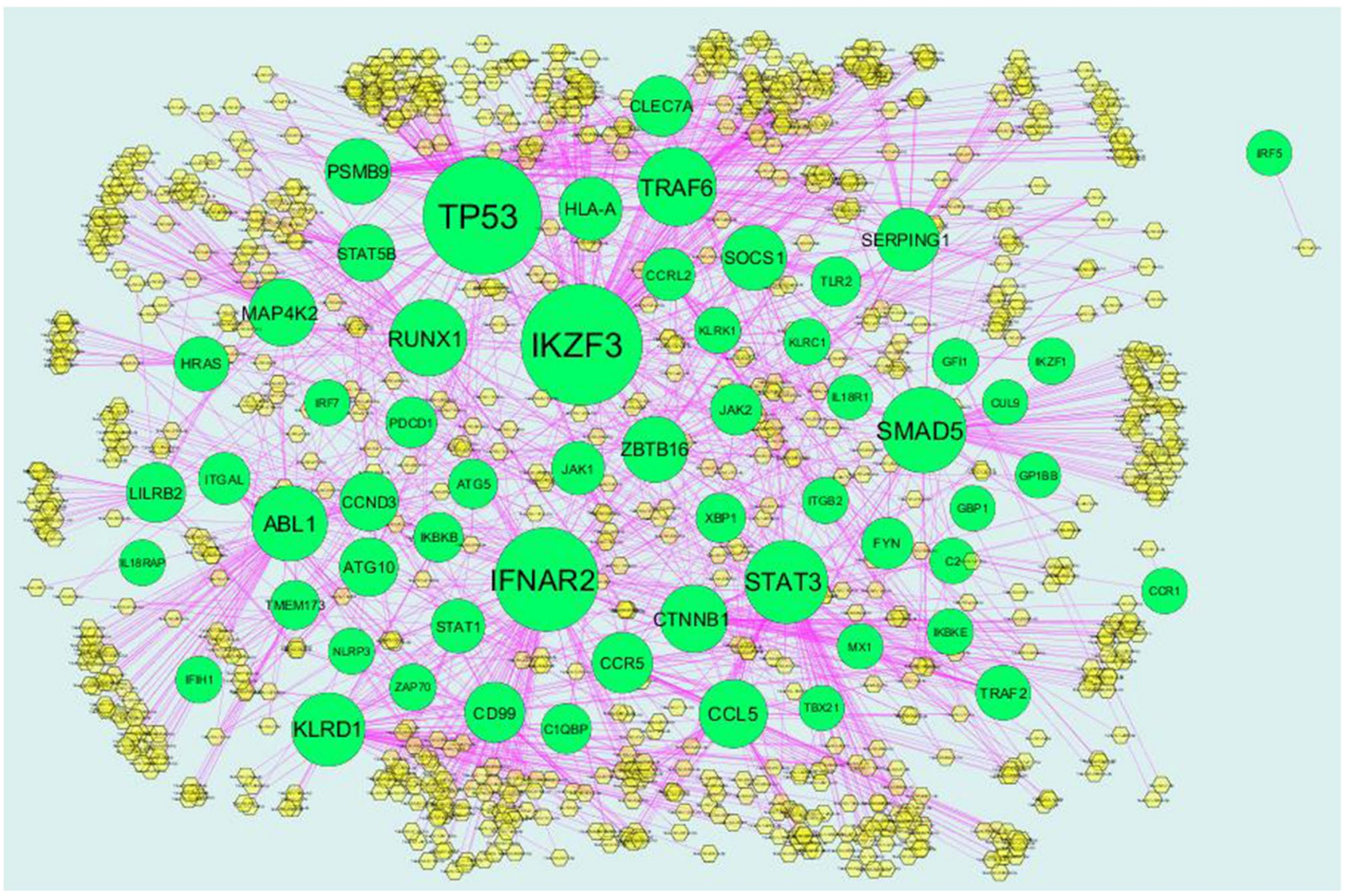

Fig. 12 The network of up regulated genes and their related miRNAs. The green circles nodes ( ) are the up regulated genes; yellow diamond nodes ( $\bigcirc)$ are the miRNAs; Pink lines (_) denotes edges (Interactions)

et al. 2012) are expressed and responsible for progression various viral infections, but our study found that these genes may important for development of SARS-CoV-2 infection. Previously reported genes such as CCR5 (Dawson et al. 2000) and TRAF6 (Tian et al. 2018) were highly expressed and involved in progression of influenza A viral infections, but these genes may be liable for advancement of SARS-CoV-2 infection. Zhivaki et al. (2017) noticed that expression of CX3CR 1 is associated in progression of respiratory syncytial virus infection, but this gene may be linked with development of SARS-CoV-2 infection. Previous studies had reported that expression of CD45RB was key for progression of sendai virus infection (Hou and Doherty 1993), but this gene may liable for advancement of SARS-CoV-2 infection. Corominas et al. (2020) showed the possible involvement of IL6R in the development of SARS-CoV-2 infection. Evidence from Chi et al. (2013) study indicated that the HLA-DQB1 expression level is down-regulated in varicella-zoster virus infection, but low expression of this gene may be associated in progression of SARS-CoV-2 infection.
Pathway enrichment analysis results for up- and downregulated gene might play important roles in the SARS$\mathrm{CoV}-2$ infection. Studies have found that over expression of enriched genes such as CCND3 (Fan et al. 2017), IRF7 (Rosenberger et al. 2017), MX1 (Pillai et al. 2016) and STAT4 (Bot et al. 2003) in influenza viral infection, but these genes may be important for progression of SARS$\mathrm{CoV}-2$ infection. JAK2 is a protein-coding gene which was first reported aberrantly expressed and plays important roles in SARS-CoV-2 infection (Wu and Yang 2020). After that, enriched up-regulated genes such as IFIH1 (Asgari et al. 2017), FYN (FYN proto-oncogene, Src family tyrosine kinase) (Kenney and Meng 2015), STAT1 (Patel et al. 2010), GZMB (granzyme B) (Loebbermann et al. 2012a, b), TRAF2 (Liu et al. 2019) and BST2 (Wang et al. 2019) were found to be involved in development of severe viral respiratory infections. Rice et al. (2016) suggested that TLR2 activity was involved in progression of pneumovirus infection, but this gene may be involved in development of SARS-CoV-2 infection. IL2RG has been shown to have an important role in adeno-associated viral infection (Hiramoto et al. 2018), but this gene may be involved in progression 
Table 7 miRNA - target gene interaction table

\begin{tabular}{|c|c|c|c|c|c|c|c|}
\hline Regulation & Target Genes & Degree & MicroRNA & Regulation & Target Genes & Degree & MicroRNA \\
\hline Up & IKZF3 & 134 & hsa-mir-6860 & Down & SKI & 210 & hsa-mir-5100 \\
\hline Up & TP53 & 130 & hsa-mir-5703 & Down & TNFRSF13C & 136 & hsa-mir-3197 \\
\hline Up & IFNAR2 & 109 & hsa-mir -4510 & Down & BCL2L11 & 122 & hsa-mir-8064 \\
\hline Up & SMAD5 & 83 & hsa-mir-6086 & Down & ICOSLG & 119 & hsa-mir-3672 \\
\hline Up & STAT3 & 80 & hsa-mir -4270 & Down & IL6R & 94 & hsa-mir-7641 \\
\hline Up & TRAF6 & 71 & hsa-mir-6745 & Down & VCAM1 & 86 & hsa-mir -4270 \\
\hline Up & RUNX1 & 67 & hsa-mir -4467 & Down & LILRA2 & 83 & hsa-mir -4780 \\
\hline Up & ABL1 & 64 & hsa-mir -4511 & Down & IRAK4 & 78 & hsa-mir-520e \\
\hline Up & KLRD1 & 60 & hsa-mir-5094 & Down & BTLA & 72 & hsa-mir-3133 \\
\hline Up & CCL5 & 49 & hsa-mir -4775 & Down & CD55 & 71 & hsa-mir-6124 \\
\hline Up & CTNNB1 & 48 & hsa-mir -4255 & Down & CCL22 & 71 & hsa-mir-5190 \\
\hline Up & MAP4K2 & 47 & hsa-mir-6131 & Down & CD44 & 70 & hsa-mir-5696 \\
\hline Up & PSMB9 & 44 & hsa-mir-3658 & Down & PDGFB & 69 & hsa-mir-6132 \\
\hline Up & ZВТВ16 & 43 & hsa-mir -4287 & Down & BCL2 & 68 & hsa-mir-184 \\
\hline Up & SOCS1 & 42 & hsa-mir -4495 & Down & TFRC & 67 & hsa-mir-6070 \\
\hline Up & HLA-A & 39 & hsa-mir-6129 & Down & PIGR & 66 & hsa-mir - 4486 \\
\hline Up & SERPING1 & 38 & hsa-mir-1262 & Down & CCL16 & 63 & hsa-mir-7703 \\
\hline Up & CCR5 & 33 & hsa-mir-3183 & Down & IFNAR1 & 61 & hsa-mir - 4430 \\
\hline Up & CLEC7A & 33 & hsa-mir -4792 & Down & TIRAP & 61 & hsa-mir -4325 \\
\hline Up & CD99 & 32 & hsa-mir-3199 & Down & ETS1 & 58 & hsa-mir-3972 \\
\hline Up & LILRB2 & 30 & hsa-mir-3941 & Down & CD59 & 56 & hsa-mir-3919 \\
\hline Up & ATG10 & 29 & hsa-mir - 4309 & Down & IKZF2 & 55 & hsa-mir-5096 \\
\hline Up & CCND3 & 29 & hsa-mir-1321 & Down & CEBPB & 54 & hsa-mir -4510 \\
\hline Up & STAT5B & 25 & hsa-mir-8485 & Down & CD209 & 54 & hsa-mir-3188 \\
\hline $\mathrm{Up}$ & TRAF2 & 21 & hsa-mir-6165 & Down & HLA-C & 53 & hsa-mir - 4660 \\
\hline Up & HRAS & 21 & hsa-mir-1268a & Down & DUSP4 & 53 & hsa-mir-6089 \\
\hline Up & STAT1 & 19 & hsa-mir-1183 & Down & CCR6 & 52 & hsa-mir - 4539 \\
\hline Up & JAK1 & 17 & hsa-mir-107 & Down & TGFBR1 & 51 & hsa-mir-8083 \\
\hline Up & CCRL2 & 16 & hsa-mir - 4469 & Down & MAPKAPK2 & 50 & hsa-mir - 4468 \\
\hline Up & ITGAL & 14 & hsa-mir-764 & Down & SLC2A1 & 50 & hsa-mir - 4448 \\
\hline Up & FYN & 14 & hsa-mir-3924 & Down & POU2F2 & 49 & hsa-mir - 4307 \\
\hline Up & JAK2 & 14 & hsa-mir-5692a & Down & LIF & 47 & hsa-mir-3655 \\
\hline Up & PDCD1 & 13 & hsa-mir-922 & Down & MBL2 & 47 & hsa-mir-3689c \\
\hline Up & C1QBP & 11 & hsa-mir - 484 & Down & HLA-B & 44 & hsa-mir-5047 \\
\hline $\mathrm{Up}$ & IKBKB & 10 & hsa-mir $-451 \mathrm{a}$ & Down & ZEB1 & 43 & hsa-mir-2113 \\
\hline Up & TLR2 & 10 & hsa-mir-105-5p & Down & RELA & 41 & hsa-mir-7515 \\
\hline Up & XBP1 & 10 & hsa-mir-320c & Down & CD46 & 39 & hsa-mir -4780 \\
\hline Up & TMEM173 & 9 & hsa-mir-5093 & Down & PAX5 & 37 & hsa-mir-6127 \\
\hline Up & ATG5 & 9 & hsa-mir-299-5p & Down & CASP3 & 36 & hsa-mir-4666b \\
\hline Up & ZAP70 & 4 & hsa-mir-631 & Down & STAT5A & 34 & hsa-mir - 4457 \\
\hline Up & IFIH1 & 4 & hsa-mir $-424-5 p$ & Down & PTAFR & 33 & hsa-mir - 4301 \\
\hline Up & KLRC1 & 4 & hsa-mir-9-5p & Down & THY1 & 32 & hsa-mir - 4269 \\
\hline Up & IKZF1 & 4 & hsa-mir-19a-3p & Down & ATG7 & 29 & hsa-mir - 4518 \\
\hline Up & KLRK1 & 3 & hsa-mir-148b-3p & Down & S1PR1 & 29 & hsa-mir-7977 \\
\hline Up & IKBKE & 3 & hsa-mir-296-5p & Down & C8A & 29 & hsa-mir-1299 \\
\hline Up & GFI1 & 3 & hsa-mir-142-3p & Down & CDH5 & 28 & hsa-mir-544a \\
\hline Up & NLRP3 & 3 & hsa-mir-223-3p & Down & GPI & 27 & hsa-mir-760 \\
\hline Up & ITGB2 & 3 & hsa-mir-146a-5p & Down & ILF3 & 27 & hsa-mir - 4314 \\
\hline Up & IL18RAP & 2 & hsa-mir $-4677-3 p$ & Down & KIT & 25 & hsa-mir -4490 \\
\hline Up & IRF7 & 2 & hsa-mir-762 & Down & GPR183 & 24 & hsa-mir-1303 \\
\hline
\end{tabular}


Table 7 (continued)

\begin{tabular}{|c|c|c|c|c|c|c|c|}
\hline Regulation & Target Genes & Degree & MicroRNA & Regulation & Target Genes & Degree & MicroRNA \\
\hline Up & $\mathrm{C} 2$ & 2 & hsa-mir-335-5p & Down & TRAF3 & 24 & hsa-mir-8085 \\
\hline Up & MX1 & 2 & hsa-mir-211-5p & Down & PTPN6 & 24 & hsa-mir -4525 \\
\hline Up & CCR1 & 2 & hsa-mir-181d-3p & Down & SELPLG & 23 & hsa-mir-1470 \\
\hline Up & CUL9 & 1 & hsa-mir-335-5p & Down & RAG1 & 23 & hsa-mir-3666 \\
\hline Up & IL18R1 & 1 & hsa-mir-124-3p & Down & HLA-DRB1 & 23 & hsa-mir-3978 \\
\hline Up & GBP1 & 1 & hsa-mir-124-3p & Down & TAL1 & 23 & hsa-mir - 4719 \\
\hline Up & TBX21 & 1 & hsa-mir-29b-3p & Down & CCL11 & 23 & hsa-mir-6077 \\
\hline Up & GP1BB & 1 & hsa-mir-26b-5p & Down & IL6 & 22 & hsa-mir-451a \\
\hline \multirow[t]{42}{*}{$\mathrm{Up}$} & IRF5 & 1 & hsa-mir-22-3p & Down & TGFBI & 22 & hsa-mir-1322 \\
\hline & & & & Down & CTSS & 22 & hsa-mir-8066 \\
\hline & & & & Down & CXCL12 & 20 & hsa-mir-886-3p \\
\hline & & & & Down & BID & 20 & hsa-mir-623 \\
\hline & & & & Down & TOLLIP & 20 & hsa-mir-6078 \\
\hline & & & & Down & CD86 & 20 & hsa-mir- 8056 \\
\hline & & & & Down & TIGIT & 20 & hsa-mir-3941 \\
\hline & & & & Down & CD3E & 20 & hsa-mir -4510 \\
\hline & & & & Down & TNFRSF9 & 19 & hsa-mir-1305 \\
\hline & & & & Down & CXCR2 & 18 & hsa-mir-588 \\
\hline & & & & Down & C6 & 18 & hsa-mir - 4310 \\
\hline & & & & Down & IL5 & 17 & hsa-mir-604 \\
\hline & & & & Down & EBI3 & 17 & hsa-mir-6069 \\
\hline & & & & Down & TCF4 & 15 & hsa-let-7e-5p \\
\hline & & & & Down & BCAP31 & 15 & hsa-mir -4514 \\
\hline & & & & Down & CD82 & 15 & hsa-mir-1470 \\
\hline & & & & Down & FCAR & 15 & hsa-mir-1976 \\
\hline & & & & Down & SELE & 14 & hsa-mir-630 \\
\hline & & & & Down & HLA-DRA & 14 & hsa-mir-3915 \\
\hline & & & & Down & IFNB1 & 13 & hsa-mir-6080 \\
\hline & & & & Down & CD9 & 13 & hsa-mir-5688 \\
\hline & & & & Down & IL10RA & 12 & hsa-mir-8064 \\
\hline & & & & Down & IL1RL2 & 12 & hsa-mir - 4301 \\
\hline & & & & Down & IL1RAP & 11 & hsa-mir-4635 \\
\hline & & & & Down & IL7 & 11 & hsa-mir-203a-3p \\
\hline & & & & Down & CD19 & 11 & hsa-mir - 466 \\
\hline & & & & Down & IFNG & 10 & hsa-mir-15b-5p \\
\hline & & & & Down & EGR2 & 10 & hsa-mir-100-5p \\
\hline & & & & Down & PTGER4 & 10 & hsa-mir-101-3p \\
\hline & & & & Down & $\mathrm{C} 1 \mathrm{~S}$ & 10 & hsa-mir-548 s \\
\hline & & & & Down & CX3CL1 & 10 & hsa-mir-5093 \\
\hline & & & & Down & CD244 & 10 & hsa-mir-5702 \\
\hline & & & & Down & $\mathrm{C} 7$ & 10 & hsa-mir-1827 \\
\hline & & & & Down & PTK2 & 9 & hsa-mir-543 \\
\hline & & & & Down & MS4A1 & 9 & hsa-mir-644a \\
\hline & & & & Down & AIRE & 9 & hsa-mir -4770 \\
\hline & & & & Down & HLA-DOB & 9 & hsa-mir-1260a \\
\hline & & & & Down & MAP4K4 & 8 & hsa-mir-520e \\
\hline & & & & Down & TRAF4 & 8 & hsa-mir - 4284 \\
\hline & & & & Down & FN1 & 8 & hsa-mir-200b-3p \\
\hline & & & & Down & STAT6 & 8 & hsa-mir-361-5p \\
\hline & & & & Down & CXCL11 & 8 & hsa-mir -4511 \\
\hline
\end{tabular}


Table 7 (continued)

\begin{tabular}{|c|c|c|c|c|c|c|}
\hline Regulation & Target Genes & MicroRNA & Regulation & Target Genes & Degree & MicroRNA \\
\hline & & & Down & EGR1 & 8 & hsa-mir-377-3p \\
\hline & & & Down & $\mathrm{C} 1 \mathrm{R}$ & 8 & hsa-mir-326 \\
\hline & & & Down & IL10 & 7 & hsa-mir-106a-5p \\
\hline & & & Down & IL12B & 7 & hsa-mir-103b \\
\hline & & & Down & PLAU & 7 & hsa-mir-23b-3p \\
\hline & & & Down & GATA3 & 7 & hsa-mir-10b-5p \\
\hline & & & Down & FCGR2A & 7 & hsa-mir -4275 \\
\hline & & & Down & AICDA & 7 & hsa-mir-6873-3p \\
\hline & & & Down & NT5E & 6 & hsa-mir $-422 \mathrm{a}$ \\
\hline & & & Down & CTLA4 & 6 & hsa-mir-3924 \\
\hline & & & Down & SPP1 & 6 & hsa-mir-299-5p \\
\hline & & & Down & BTK & 6 & hsa-mir-1253 \\
\hline & & & Down & MIF & 6 & hsa-mir-320a \\
\hline & & & Down & IL13 & 5 & hsa-let-7i-5p \\
\hline & & & Down & CD34 & 5 & hsa-mir-106b-5p \\
\hline & & & Down & IL4 & 5 & hsa-mir-429 \\
\hline & & & Down & RORC & 5 & hsa-mir-148b-3p \\
\hline & & & Down & CTSC & 5 & hsa-mir-199a-5p \\
\hline & & & Down & CD40 & 5 & hsa-mir-503-5p \\
\hline & & & Down & IL2 & 5 & hsa-mir-181c-5p \\
\hline & & & Down & TCF7 & 4 & hsa-mir-22-3p \\
\hline & & & Down & ICAM5 & 4 & hsa-mir $-4707-5 p$ \\
\hline & & & Down & CD83 & 4 & hsa-mir-122-5p \\
\hline & & & Down & HLA-DQA1 & 4 & hsa-mir -4673 \\
\hline & & & Down & IL4R & 4 & hsa-mir-331-3p \\
\hline & & & Down & MAPK11 & 3 & hsa-let-7a-5p \\
\hline & & & Down & TNFSF12 & 3 & hsa-mir-17-5p \\
\hline & & & Down & CCL7 & 3 & hsa-mir-135b-3p \\
\hline & & & Down & IL12A & 3 & hsa-mir-10a-5p \\
\hline & & & Down & CSF1R & 3 & hsa-mir-155-5p \\
\hline & & & Down & CFI & 3 & hsa-mir-181a-5p \\
\hline & & & Down & IL3 & 3 & hsa-mir $-452-5 p$ \\
\hline & & & Down & NFIL3 & 3 & hsa-mir-183-5p \\
\hline & & & Down & ICAM3 & 3 & hsa-mir-3943 \\
\hline & & & Down & EOMES & 3 & hsa-mir-7855-5p \\
\hline & & & Down & PTPN22 & 3 & hsa-mir-624-3p \\
\hline & & & Down & LGALS3 & 3 & hsa-mir-744-5p \\
\hline & & & Down & CCL19 & 3 & hsa-mir-148b-3p \\
\hline & & & Down & IL17A & 3 & hsa-mir-16-1-3p \\
\hline & & & Down & $\mathrm{CD} 22$ & 2 & hsa-mir-19a-3p \\
\hline & & & Down & CCL20 & 2 & hsa-mir-21-5p \\
\hline & & & Down & CCL26 & 2 & hsa-mir-25-3p \\
\hline & & & Down & DEFB4A & 2 & hsa-mir-26b-5p \\
\hline & & & Down & CXCR1 & 2 & hsa-mir-335-5p \\
\hline & & & Down & MME & 2 & hsa-mir-1-3p \\
\hline & & & Down & VTN & 2 & hsa-mir-26b-5p \\
\hline & & & Down & LILRB5 & 2 & hsa-mir-128-3p \\
\hline & & & Down & IL13RA1 & 2 & hsa-mir-143-3p \\
\hline & & & Down & SIGIRR & 2 & hsa-mir-149-5p \\
\hline & & & Down & C14orf166 & 2 & hsa-mir-331-3p \\
\hline
\end{tabular}


Table 7 (continued)

\begin{tabular}{|c|c|c|c|c|c|c|c|}
\hline Regulation & Target Genes & Degree & MicroRNA & Regulation & Target Genes & Degree & MicroRNA \\
\hline & & & & Down & CD8A & 2 & hsa-mir-196b-5p \\
\hline & & & & Down & IRF8 & 2 & hsa-mir-646 \\
\hline & & & & Down & FCGR2B & 1 & hsa-mir-18a-5p \\
\hline & & & & Down & NOS2 & 1 & hsa-mir-26a-5p \\
\hline & & & & Down & RELB & 1 & hsa-mir-26b-5p \\
\hline & & & & Down & CXCL13 & 1 & hsa-mir-26b-5p \\
\hline & & & & Down & BATF3 & 1 & hsa-mir-26b-5p \\
\hline & & & & Down & ICAM4 & 1 & hsa-mir-93-5p \\
\hline & & & & Down & IL23A & 1 & hsa-mir-10a-5p \\
\hline & & & & Down & ARHGDIB & 1 & hsa-mir-34a-5p \\
\hline & & & & Down & LTA & 1 & hsa-mir-34a-5p \\
\hline & & & & Down & ICAM2 & 1 & hsa-mir-125b-5p \\
\hline & & & & Down & CR2 & 1 & hsa-mir-132-3p \\
\hline & & & & Down & B3GAT1 & 1 & hsa-mir-132-3p \\
\hline & & & & Down & IDO1 & 1 & hsa-mir-153-3p \\
\hline & & & & Down & MASP1 & 1 & hsa-mir-153-3p \\
\hline & & & & Down & CD80 & 1 & hsa-mir-146a-5p \\
\hline & & & & Down & HLA-DPA1 & 1 & hsa-mir-155-5p \\
\hline & & & & Down & TAGAP & 1 & hsa-mir-374a-5p \\
\hline & & & & Down & CD79A & 1 & hsa-mir-335-5p \\
\hline & & & & Down & FCGRT & 1 & hsa-mir-335-5p \\
\hline & & & & Down & HLA-DMA & 1 & hsa-mir-335-5p \\
\hline & & & & Down & KLRB1 & 1 & hsa-mir-335-5p \\
\hline & & & & Down & LCK & 1 & hsa-mir-335-5p \\
\hline & & & & Down & PPBP & 1 & hsa-mir-335-5p \\
\hline & & & & Down & CCL15 & 1 & hsa-mir-335-5p \\
\hline & & & & Down & CCL24 & 1 & hsa-mir-335-5p \\
\hline & & & & Down & LILRA1 & 1 & hsa-mir-335-5p \\
\hline & & & & Down & LILRA3 & 1 & hsa-mir-335-5p \\
\hline & & & & Down & PLA2G2E & 1 & hsa-mir-335-5p \\
\hline & & & & Down & IL22 & 1 & hsa-mir-335-5p \\
\hline & & & & Down & IL27 & 1 & hsa-mir-335-5p \\
\hline & & & & Down & MBP & 1 & hsa-mir-127-5p \\
\hline & & & & Down & IL20 & 1 & hsa-mir-624-3p \\
\hline
\end{tabular}

Degree - No of miRNA interact with target gene. We taken any one miRNA in table

of SARS-CoV-2 infection. Reported enriched up-regulated genes such as STAT3 (Mizutani et al. 2004) and HLA-A (Ohno et al. 2009) contributes to the progression of SARS coronavirus infection, but this gene may be involved in SARS-CoV-2 infection. Several studies have reported that enriched genes such as STAT5B (Mukherjee et al. 2014), SOCS1 (Zheng et al. 2015), CCR1 (Miller et al. 2006) and CCL5 (Sali mi et al. 2017) were highly expressed in respiratory syncytial virus infection, but elevated expression these genes may be involved in development of SARS-CoV-2 infection. Increasing evidence shows that the enriched genes such as IFNAR2 (Romporn et al. 2013), TBX21 (Zhu et al. 2015), GBP1 (Anderson et al. 1999), IRF5 (Vandenbroeck et al. 2011) and IFI35 (Estrabaud et al. 2015) were over expressed in various viral infections, but high expression of these genes may be involved in infection of SARS-CoV-2 infection. Novel biomarkers such as IKBKE (inhibitor of nuclear factor kappa B kinase subunit epsilon), TP53, CD247, IL18RAP, IL18R1, HRAS (HRas proto-oncogene, GTPase), PSMB9, IKBKB (inhibitor of nuclear factor kappa B kinase subunit beta), ITGB2 and LTB4R were highly expressed and might be involved in progression of SARSCoV-2 infection. Sanders et al. (2001) revealed that NOS2 was down-regulated in rhinovirus infection, but this gene may be involved in development of SARS-CoV-2 infection. The enriched down-regulated genes found in this study 


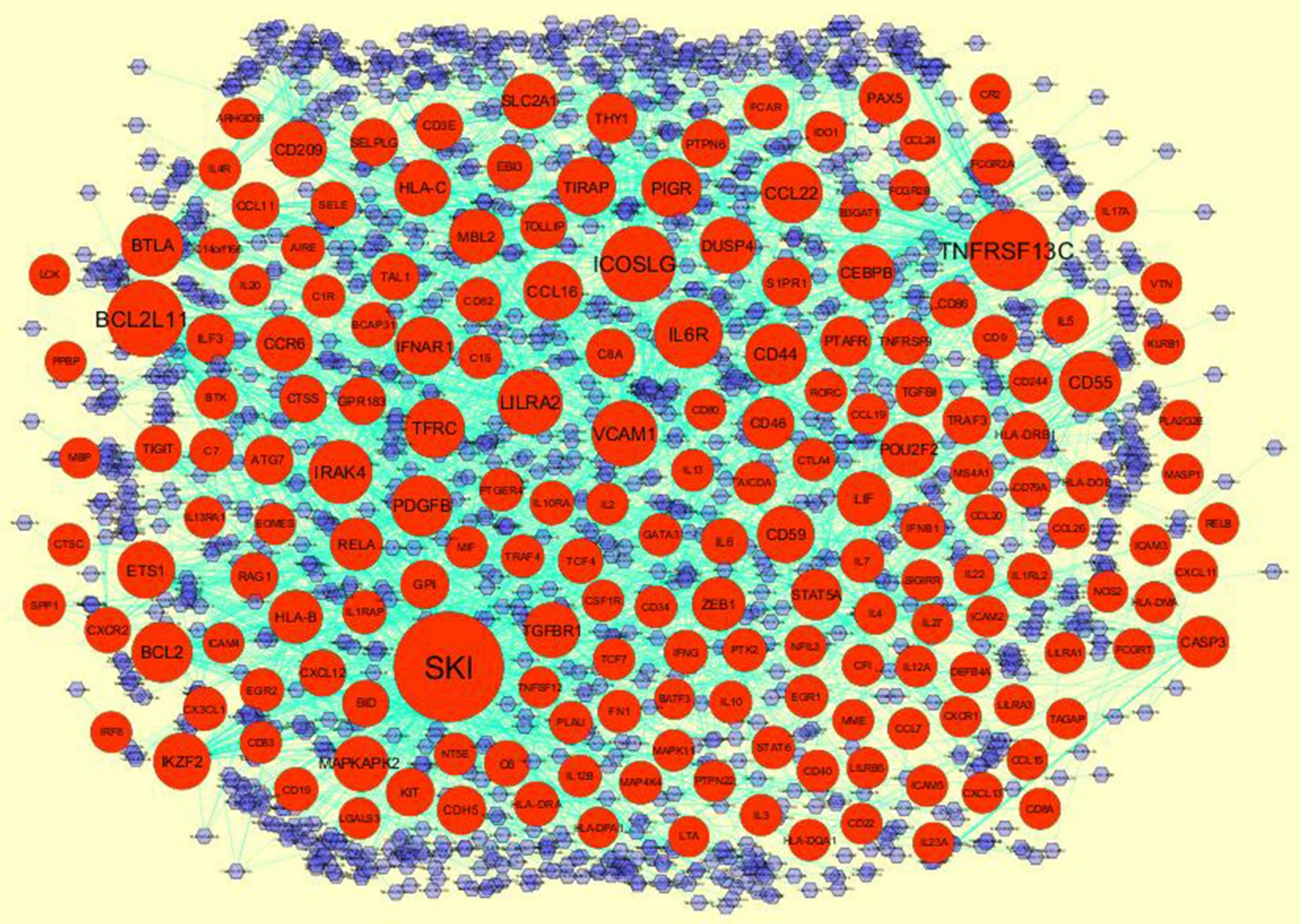

Fig. 13 The network of down regulated genes and their related miRNAs. The red circles nodes (3) are the down regulated genes; blue diamond nodes (O) are the miRNAs; Sku blue lines (-) denotes edges (Interactions)

include IL10 (Loebbermann et al. 2012a, b), IL13 (Castilow et al. 2008), IL21 (Antunes et al. 2019), CCR6 (Shi et al. 2017), CXCL13 (Alturaiki et al. 2018), CCL20 (Shi et al. 2017), IL19 (Ermers et al. 2011), IL20 (Ermers et al. 2011), CD40 (Harcourt et al. 2003a, b), IL2 (Noma et al. 1996), IL3 (Bertrand et al. 2015), IL4 (Puthothu et al. 2006), IL9 (Dodd et al. 2009) and STAT6 (Srinivasa et al. 2016) were responsible for progression of respiratory syncytial virus infection, but these genes may be linked with progression of SARS-CoV-2 infection. Many previous studies have confirmed the roles of enriched down-regulated genes such as IL12B (Mueller et al. 2004), TNFRSF9 (Rodriguez et al. 2019), TNFRSF14 (Soroosh et al. 2014), IL17F (Wang et al. 2016), CCR8 (Calado et al. 2010), CCL18 (Malhotra et al. 2019), CCL22 (Yang et al. 2012), CXCL11 (Pineda-Tenor et al. 2014), CX3CL1 (Bertin et al. 2014), CXCL12 (Durrant et al. 2014), CCR10 (Nakayama et al. 2002), IFNA2 (Chen et al. 2017), IFNB1 (Gagné et al. 2017), IL7 (GoldenMason et al. 2006), IL26 (Miot et al. 2015), CXCR1 (Xu et al. 2016), CEBPB (CCAAT enhancer binding protein beta) (Liu et al. 2009), ETS1 (Posada et al. 2000), STAT5A (Warby et al. 2003), THY1 (Lu et al. 2011), IL16 (Caufour et al. 2001), HLA-B (Martin et al. 2007), HLA-C (Apps et al. 2013), HLA-DPA1 (Wasityastuti et al. 2016), HLADPB1 (Lambert et al. 2015), HLA-DQA1 (Tibbs et al. 1996), HLA-DRB1 (Chi et al. 2013), PSMB10 (Deng et al. 2019), BCL2 (Zuckerman et al. 2001), TOLLIP (toll interacting protein) ( $\mathrm{Li}$ et al. 2016a, b), VCAM1 (Koraka et al. 2004), RAG1 (Winkler et al. 2017), IRF8 (Terry et al. 2015), EBI3 (Gehlert et al. 2004), EGR1 (Baer et al. 2016), IL27 (Swaminathan et al. 2013) and BID (BH3 interacting domain death agonist) (Hsu et al. 2003) were linked with development of various viral infections, but these genes may be associated with advancement of SARS-CoV-2 infection. Previous investigation demonstrated that enriched downregulated genes such as IL17A (Wang et al. 2016), CCL11 (Suryadevara et al. 2013), CCL19 (Fleming-Canepa et al. 2011), XCR1 (Fossum et al. 2015), IFNAR1 (Lin et al. 2014), IL22 (Kumar et al. 2013), LTA (lymphotoxin alpha) (Morales-García et al. 2012), IL5 (Gorski et al. 2013), EGR2 
Table $8 \mathrm{TF}$ - target gene interaction table

\begin{tabular}{|c|c|c|c|c|c|c|c|}
\hline Regulation & $\mathrm{TF}$ & Degree & Target Gene & Regulation & $\mathrm{TF}$ & Degree & Target Gene \\
\hline Up & FOXC1 & 46 & JAK1 & Down & FOXC1 & 127 & KLRF2 \\
\hline Up & GATA2 & 31 & TRAF6 & Down & GATA2 & 102 & CD1A \\
\hline Up & YY1 & 25 & CLEC7A & Down & YY1 & 75 & TNFRSF4 \\
\hline Up & CREB1 & 22 & STAT1 & Down & FOXL1 & 63 & MME \\
\hline Up & TFAP2A & 21 & IKZF1 & Down & NFKB1 & 61 & CXCL12 \\
\hline Up & PPARG & 20 & IRF7 & Down & USF2 & 48 & DEFB1 \\
\hline Up & NFKB 1 & 20 & TRAF2 & Down & SRF & 48 & STAT5A \\
\hline Up & E2F1 & 19 & PSMB9 & Down & CREB1 & 46 & TNFRSF10C \\
\hline Up & RELA & 18 & IFI35 & Down & TP53 & 45 & $\mathrm{C} 1 \mathrm{~S}$ \\
\hline Up & USF2 & 17 & LTB4R & Down & STAT3 & 45 & IL26 \\
\hline Up & SREBF1 & 16 & CCND3 & Down & PPARG & 43 & ICAM4 \\
\hline Up & HINFP & 15 & GP1BB & Down & JUN & 42 & FCGRT \\
\hline Up & JUN & 15 & TLR2 & Down & $\mathrm{E} 2 \mathrm{~F} 1$ & 42 & GATA3 \\
\hline Up & FOXL1 & 12 & LCP2 & Down & HINFP & 40 & BCL2L11 \\
\hline Up & BRCA1 & 11 & CD99 & Down & TFAP2A & 38 & EOMES \\
\hline Up & NFIC & 11 & FYN & Down & NFIC & 38 & IFNA2 \\
\hline Up & SRF & 11 & IL18RAP & Down & MEF2A & 31 & CTSS \\
\hline Up & GATA3 & 9 & CX3CR1 & Down & NFYA & 29 & GPI \\
\hline Up & PRDM1 & 9 & GNLY & Down & TEAD1 & 28 & LTA \\
\hline Up & POU2F2 & 9 & SLAMF7 & Down & HOXA5 & 28 & PPBP \\
\hline Up & USF1 & 8 & ATG5 & Down & MAX & 27 & IL12B \\
\hline Up & FOS & 8 & CCL5 & Down & SREBF2 & 26 & CD80 \\
\hline Up & TEAD1 & 8 & CCR1 & Down & SREBF1 & 26 & DEFB4A \\
\hline Up & TFAP2C & 8 & CEACAM1 & Down & JUND & 26 & HLA-DPB1 \\
\hline Up & ELK4 & 8 & GBP1 & Down & TFAP2C & 26 & HLA-DQA1 \\
\hline Up & HOXA5 & 8 & GZMB & Down & RUNX2 & 25 & $\mathrm{C} 1 \mathrm{R}$ \\
\hline Up & E2F6 & 8 & HRAS & Down & ARID3A & 24 & SKI \\
\hline Up & HNF4A & 8 & IFNAR2 & Down & HNF4A & 23 & CR2 \\
\hline Up & RUNX2 & 8 & KLRK1 & Down & ZNF354C & 22 & $\mathrm{C} 8 \mathrm{~A}$ \\
\hline Up & NFYA & 7 & ATG10 & Down & NR3C1 & 22 & FCGR2B \\
\hline Up & SREBF2 & 7 & C1QBP & Down & IRF2 & 22 & XCR1 \\
\hline Up & MAX & 7 & CCRL2 & Down & PRDM1 & 21 & IL13 \\
\hline Up & CEBPB & 7 & CD247 & Down & FOS & 20 & CCL15 \\
\hline Up & PRRX2 & 7 & CTNNB1 & Down & USF1 & 20 & IRAK4 \\
\hline Up & ZNF354C & 7 & GFI1 & Down & FOXA1 & 19 & ITLN1 \\
\hline Up & MEF2A & 7 & IL18R1 & Down & PRRX2 & 19 & TLR9 \\
\hline Up & ARID3A & 6 & ABL1 & Down & STAT1 & 18 & LCK \\
\hline Up & ESR1 & 6 & $\mathrm{C} 2$ & Down & SP1 & 16 & CD82 \\
\hline Up & PDX1 & 6 & HLA-A & Down & ELK4 & 16 & PAX5 \\
\hline Up & NR3C1 & 6 & ITGAL & Down & NKX3-2 & 16 & PLA2G2E \\
\hline Up & KLF5 & 6 & ITGB2 & Down & KLF5 & 15 & ICOSLG \\
\hline Up & ELK1 & 6 & JAK2 & Down & ELK1 & 15 & IDO1 \\
\hline Up & SP1 & 6 & MAP4K2 & Down & BRCA1 & 15 & LGALS3 \\
\hline Up & EGR1 & 6 & MX1 & Down & ESR1 & 15 & MASP2 \\
\hline Up & EN1 & 6 & SMAD5 & Down & TP63 & 12 & CD19 \\
\hline Up & IRF2 & 5 & RUNX1 & Down & PAX2 & 12 & CD48 \\
\hline Up & PAX2 & 5 & STAT5B & Down & NR2F1 & 12 & CEBPB \\
\hline Up & NR2F1 & 4 & CUL9 & Down & E2F6 & 11 & CD8A \\
\hline Up & FOXF2 & 4 & IKBKE & Down & EN1 & 11 & IL22 \\
\hline Up & TP63 & 4 & IKZF3 & Down & SPIB & 11 & PTAFR \\
\hline Up & FOXA1 & 4 & IL2RG & Down & FOXF2 & 10 & SELE \\
\hline
\end{tabular}


Table 8 (continued)

\begin{tabular}{llllllll}
\hline Regulation & TF & Degree & Target Gene & Regulation & TF & Degree & Target Gene \\
\hline Up & NRF1 & 4 & MAP4K1 & Down & SOX10 & 9 & POU2F2 \\
Up & JUND & 4 & NLRP3 & Down & SRY & 8 & EDNRB \\
Up & SPIB & 4 & ZBTB16 & Down & NRF1 & 8 & IFNAR1 \\
Up & REL & 3 & IKZF3 & Down & SOX17 & 7 & TNFRSF14 \\
Up & NKX3-1 & 3 & IRF5 & Down & SOX5 & 6 & BTK \\
Up & SOX5 & 3 & KLRD1 & Down & NKX2-5 & 6 & PIGR \\
Up & SOX10 & 3 & STAT3 & Down & ELF5 & 5 & BATF3 \\
Up & MYB & 3 & JAK2 & Down & NFATC2 & 5 & IL4 \\
Up & SRY & 2 & KLRK1 & Down & PDX1 & 5 & ITGAE \\
Up & ELF5 & 2 & TLR2 & Down & NFYB & 3 & PTPN6 \\
Up & NFIL3 & 1 & CX3CR1 & Down & ZFX & 3 & THY1 \\
Up & NR4A2 & 1 & C2 & Down & NR4A2 & 2 & CSF2 \\
Up & FEV & 1 & ATG5 & Down & FEV & 2 & IL23A \\
Up & FOXI1 & 1 & CCL5 & Down & MYB & 2 & MIF \\
Up & NKX3-2 & 1 & GZMB & Down & HNF1B & 2 & SPP1 \\
Up & E2F4 & 1 & IKZF1 & Down & REL & 2 & STAT6 \\
Up & TFCP2L1 & 1 & LCP2 & Down & ESR2 & 1 & CCL22 \\
Up & NR2E3 & 1 & HLA-A & Down & FOXI1 & 1 & CD209 \\
Up & GATA1 & 1 & HLA-A & Down & FOXD1 & 1 & HLA-DOB \\
Up & HNF1B & 1 & IL18R1 & Down & ESRRB & 1 & ICAM2 \\
Up & SOX17 & 1 & LILRA6 & Down & NR2E3 & 1 & IL2 \\
& & & & Down & E2F4 & 1 & S1PR1 \\
\hline & & & & Down & GATA1 & 1 & PDGFB \\
\hline
\end{tabular}

Degree-No of TF interact with target gene. We taken any one TF in table

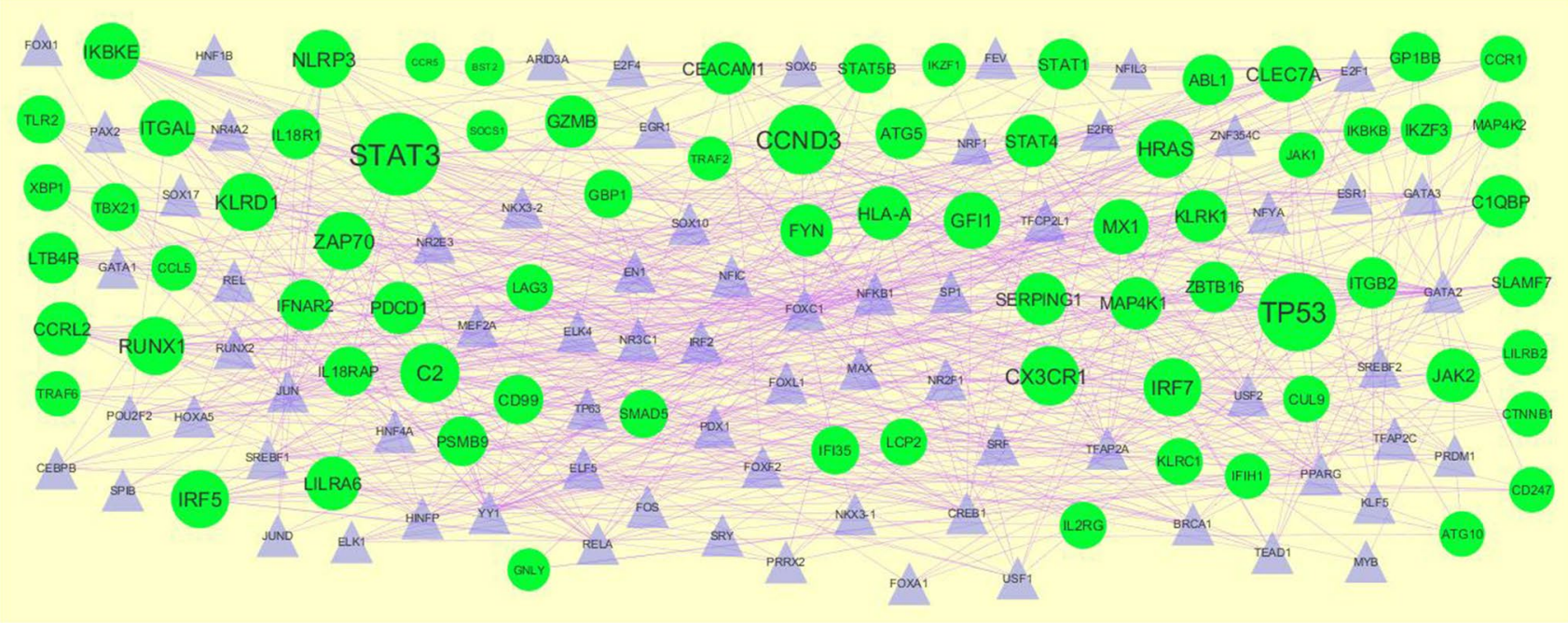

Fig. 14 The network of up regulated genes and their related TFs. The green circles nodes ( $\mathrm{O}$ ) are the up regulated genes; Blue triangle nodes ( ) are the TFs; Purple line (-) denotes edges (Interactions)

(Du et al. 2014), RAG2 (Wu et al. 2010), CASP3 (Takahashi et al. 2013), S1PR1 (Zhao et al. 2019), CD80 (Lumsden et al. 2000), CD86 (Lumsden et al. 2000) and CD44 (Liu et al. 2014) were key for advancement of influenza virus infection, but these genes may be involved in progression of SARS-CoV-2 infection. Enriched down-regulated genes such as CCL7 (Girkin et al. 2015) and CXCR2 (Nagarkar et al. 2009) have been reported to be associated with 


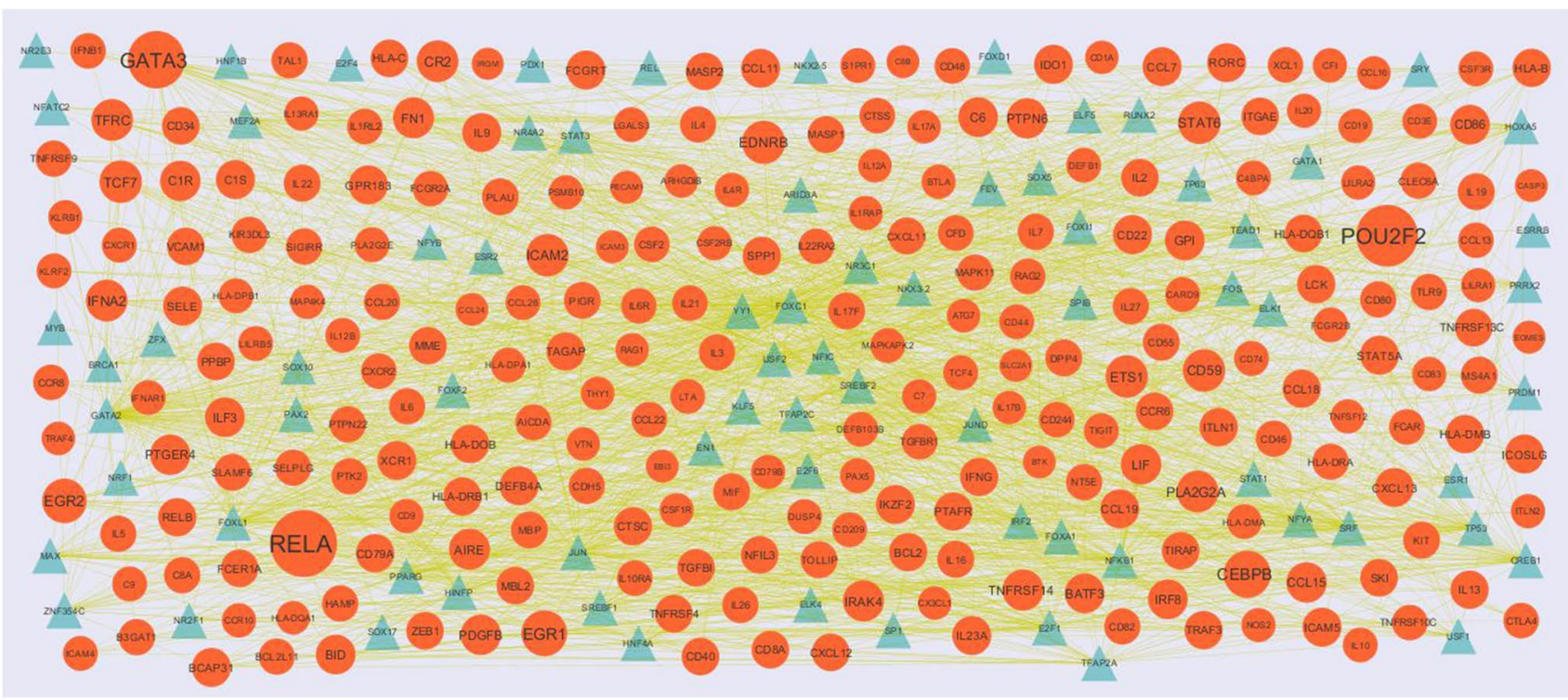

Fig. 15 The network of down regulated genes and their related TFs. The Red circles nodes ( 0 ) are the down regulated genes; Blue triangle nodes $(\hat{A})$ are the TFs; Yelow line $(-)$ denotes edges (Interactions)
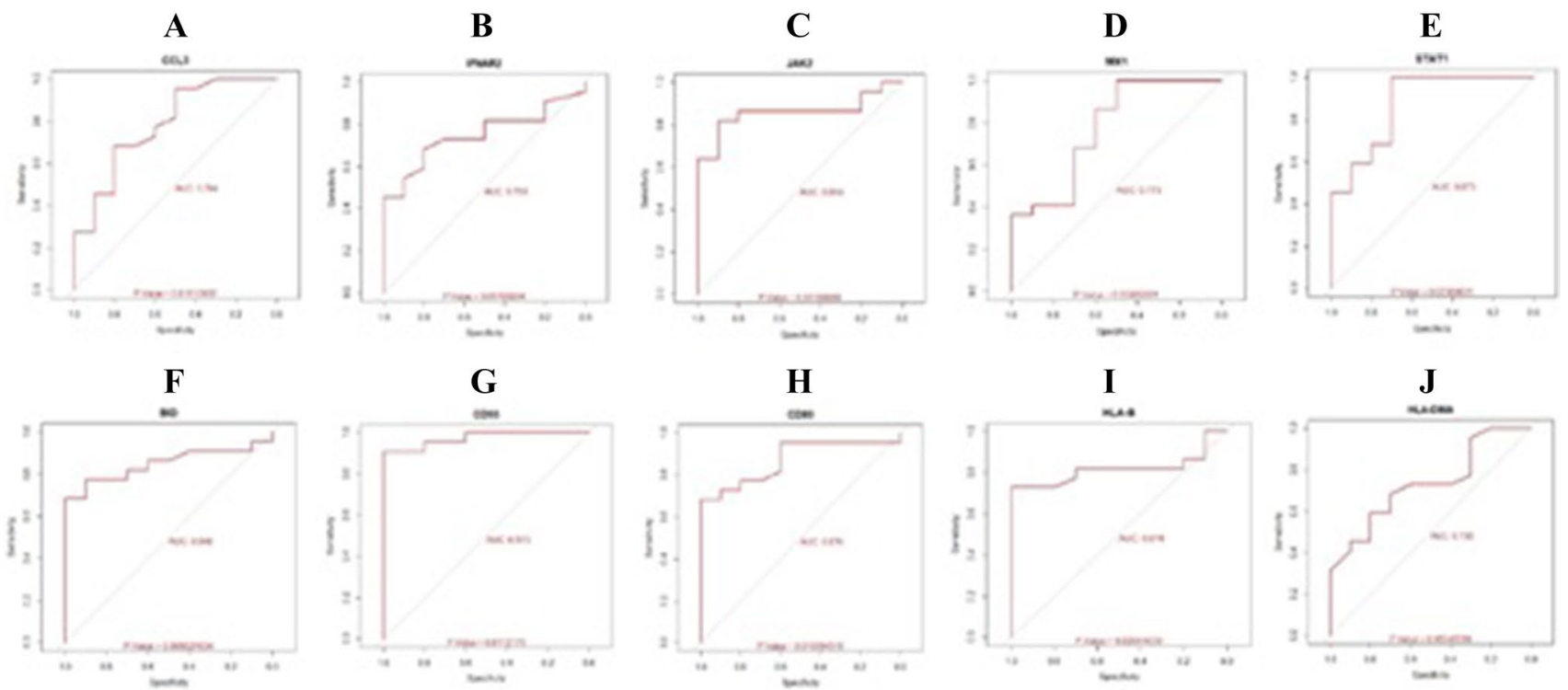

Fig. 16 ROC curve validated the sensitivity, specificity of hub genes as a predictive biomarker for SARS-CoV-2 diagnosis. a CCL5 b IFNAR2 c JAK2 d MX1 e STAT1 f BID g CD55 h CD80 i HAL-B j HLA-DMA

rhinovirus $1 \mathrm{~B}$ infection, but these genes may be responsible for infection of SARS-CoV-2. Accumulating evidence shows that enriched genes such as IFNG (interferon gamma) (Sainz et al. 2004) and TRAF3 (Siu et al. 2009) were low expressed in SARS-CoV, but decreased expression of these genes may be key for progression of SARS-CoV-2 infection. Conti et al. (2020) showed that IL6 was liable for progression of SARS-CoV-2 infection. Novel biomarkers such as IL10RA, IL12A, IL13RA1, PDGFB (platelet-derived growth factor subunit B), TNFSF12, IL17B, TNFRSF10C, CCL26, TNFRSF4, IL22RA2, CCL15, CCL16, CCL24, XCL1, KIT (KIT proto-oncogene, receptor tyrosine kinase), CCL13, PPBP (pro-platelet basic protein), IL23A, TGFBR1, LIF (LIF interleukin 6 family cytokine), CSF1R, CSF2, CSF2RB, CSF3R, TNFRSF13C, IL1RAP, IL4R, AICDA (activation-induced cytidinedeaminase), PTPN6, PIGR (polymeric immunoglobulin receptor), GATA3, PTAFR (platelet activating factor receptor), IL1RL2, PTK2, FN1, 
DUSP4, RELA (RELA proto-oncogene, NF-kA subunit), RELB (RELB proto-oncogene, NF-kB subunit), LCK (LCK proto-oncogene, Src family tyrosine kinase), IRAK4, RORC (RAR-related orphan receptor C), BCL2L11 and PLA2G2A were low expressed and might be involved in progression of SARS-CoV-2 infection.

$\mathrm{GO}$ enrichment analysis results for up- and down-regulated gene might play important roles in the SARS-CoV-2 infection. Enriched up-regulated genes such as ATG5 (Guévin et al. 2010), PDCD1 (Nasi et al. 2013), ABL1 (García et al. 2012), CD99 (Tochikura et al. 2003), LILRB2 (Alaoui et al. 2018), LAG3 (Tian et al. 2015), SERPING1 (Sanfilippo et al. 2017), XBP1 (Sharma et al. 2017), CTNNB1 (Tucci et al. 2013), RUNX1 (Zhao et al. 2016), SLAMF7 (O'Connell et al. 2019), ITGAL (integrin subunit alpha L) (Xu et al. 2018) and CEACAM1 (Hirai et al. 2010) appeared to be related in various types of viral infections, but these genes may be responsible for progression of SARS-CoV-2 infection. Hu et al. (2017) observed that high expression of C1QBP was liable for progression of respiratory syncytial viral infection, but elevated expression this gene may be associated with advancement of SARS-CoV-2 infection. Evidence demonstrated that high expression of enriched genes such as KLRD1 (Bongen et al. 2018) and NLRP3 (Pothlichet et al. 2013) were important for progression of influenza virus infection, but increased expression of these genes may be involved in advancement of SARSCoV-2 infection. Novel biomarkers such as KLRK1, IKZF3, ZBTB16, CLEC7A, C2 (complement C2), IKZF1, LCP2, KLRC1, GFI1, CCRL2 and MAP4K2 were highly expressed and might be involved in progression of SARS-CoV-2 infection. Studies have reported that low expression of enriched genes such as IRGM (immunity-related GTPase M) (Hansen et al. 2017), MASP1 (El Saadany et al. 2011), CD244 (Raziorrouh et al. 2010), MBL2 (Spector et al. 2010), CD46 (Gaggar et al. 2003), C4A (Imakiire et al. 2012), C9 (Kim et al. 2013), ZEB1 (Lacher et al. 2011), ICAM2 (Wang et al. 2009), BTLA (B and T lymphocyte associated) (Cai et al. 2013), CD1A (Sacchi et al. 2007), CD19 (Zehender et al. 1997), ICAM5 (Wei et al. 2016), CD34 (Fahrbach et al. 2007), CD48 (Ezinne et al. 2014), CD59 (Amet et al. 2012), CD74 (Le Noury et al. 2015) and DEFB1 (Estrada-Aguirre et al. 2014) were linked with development of various viral infections, but low expression of these genes may be liable for progression of SARS-CoV-2 infection. Recent studies reported that enriched genes such as IDO1 (Fox et al. 2015), CD55 (Li et al. 2016), PTPN22 (Crabtree et al. 2016), FCGR2A (Maestri et al. 2016), CARD9 (Uematsu et al. 2015), MIF (macrophage migration inhibitory factor) (Arndt et al. 2002) and PLAU (plasminogen activator, urokinase) (Sidenius et al. 2000) were low expressed in influenza virus infection, but decrease expression of these genes may be key for progression of SARS-CoV-2 infection. Low expression of genes such as PECAM1 (Wang et al. 1998), TLR9 (Shafique et al. 2012) and CTLA4 (Ayukawa et al. 2004) were observed in respiratory syncytial virus infection, but decrease expression these genes may be associated with progression of SARS-CoV-2 infection. Chen et al. (2017) demonstrated CD83 was important for progression of respiratory syndrome virus, but decrease expression of this gene may be linked with advancement of SARS-CoV-2 infection. Many studies have reported the enriched down-regulated gene such as CD209 (Chan et al. 2010), DPP4 (Letko et al. 2018), ICAM3 (Chan et al. 2007), CD9 (Earnest et al. 2017) and MASP2 (Wang et al. 2009) were liable for advancement of SARS-CoV, but these genes may be linked with progression of SARS-CoV-2 infection. Treon et al. (2020) indicated that low expression of BTK (Bruton tyrosine kinase) was key for progression of SARS-CoV-2 infection. Novel biomarkers such as HLA-DMA, HLA-DMB, HLA-DOB, HLA-DRA, CTSC (cathepsin C), PTGER4, CFD (complement factor D), SLAMF6, FCER1A, FCGR2B, C1R, C1S, C4BPA, C6, C7, C8A, C8B, TAL1, KLRB1, SELE (selectin E), GPI (glucose-6-phosphate isomerase), ICOSLG (inducible T cell costimulator ligand), LILRA1, LILRA2, VTN (vitronectin), CLEC6A, ATG7, ICAM4, AIRE (autoimmune regulator), GPR183, CFI, CR2, LGALS3, TFRC (transferrin receptor), CD3E, CD8A, TIGIT (T cell immunoreceptor with Ig and ITIM domains), MS4A1, TIRAP (TIR domain containing adaptor protein), CD79A, CD79B, PAX5, HAMP (hepcidin antimicrobial peptide), MAPK11, CTSS (cathepsin S), MBP (myelin basic protein), ITGAE (integrin subunit alpha E), FCGRT (Fc fragment of IgG receptor and transporter), MME (membrane metalloendopeptidase), NT5E, CDH5, DEFB103B, DEFB4A and TRAF4 were low expressed and might be involved in progression of SARS-CoV-2 infection.

Construction of PPI network of up- and down-regulated genes might be helpful for understanding the relationship of developmental SARS-CoV-2 infection. Desai et al. (2018) showed that BATF3 was involved in progression of respiratory poxvirus infection, but this gene may be key for development of SARS-CoV-2 infection. Novel biomarker ILF3 was low expressed and might be involved in progression of SARS-CoV-2 infection.

A target gene-miRNA regulatory and target gene-TF regulatory network for up- and down-regulated genes were generated to determine the key target genes and provide valuable information for the analysis of cellular functions and biological processes in SARS-CoV-2 infection. SMAD5 was highly expressed in SARS-CoV-2 infection and might be consider as novel biomarker. Novel biomarkers such as SKI (SKI proto-oncogene) and KLRF2 were low expressed and might be involved in progression of SARS-CoV-2 infection. 


\section{Conclusion}

It in earnestly hoped that this research will help in enhancing attempts to further understand the molecular characteristics of SARS-CoV-2 infection progression. CCL5, IFNAR2, JAK2, MX1, STAT1, BID, CD55, CD80, HAL-B and HLADMA may be used as biomarkers and therapeutic targets in patients with SARS-CoV-2 infection. This research, it is hoped promote ultimate molecularly targeted therapies for SARS-CoV-2 infection and provide acceptable local control and survival.

Acknowledgements We thank Eugenia Ong, Experimental Therapeutics Centre, Agency for Science, Technology and Research, Singapore, Singapore, very much, the author who deposited their microarray dataset, E-MTAB-8871, into the public ArrayExpress database.

Author contributions Basavaraj Vastrad participated in writing original draft and investigation, Chanabasayya Vastrad performed software, supervision, formal analysis and validation. Anandkumar Tengli performed editing and reviewing the manuscript.

Availability of data and materials The datasets supporting the conclusions of this article are available in the ArrayExpress (https://www. ebi.ac.uk/arrayexpress) repository. [(E-MTAB-8871) (https://www.ebi. ac.uk/arrayexpress/experiments/E-MTAB-8871/)].

\section{Compliance with ethical standards}

Conflict of interest The authors declare that they have no conflict of interest.

Ethical approval This article does not contain any studies with human participants or animals performed by any of the authors.

Informed consent No informed consent, because this study does not contain human or animals participants.

Consent for publication Not applicable.

\section{References}

Alaoui L, Palomino G, Zurawski S, Zurawski G, Coindre S, Dereuddre-Bosquet N, Lecuroux C, Goujard C, Vaslin B, Bourgeois C et al (2018) Early SIV and HIV infection promotes the LILRB2/ MHC-I inhibitory axis in cDCs. Cell Mol Life Sci 75(10):18711887. https://doi.org/10.1007/s00018-017-2712-9

Alturaiki W, McFarlane AJ, Rose K, Corkhill R, McNamara PS, Schwarze J, Flanagan BF (2018) Expression of the B cell differentiation factor BAFF and chemokine CXCL13 in a murine model of respiratory syncytial virus infection. Cytokine 110:267-271. https://doi.org/10.1016/j.cyto.2018.01.014

Amet T, Ghabril M, Chalasani N, Byrd D, Hu N, Grantham A, Liu Z, Qin X, He JJ, Yu Q (2012) CD59 incorporation protects hepatitis $\mathrm{C}$ virus against complement-mediated destruction. Hepatology 55(2):354-363. https://doi.org/10.1002/hep.24686

Anderson SL, Carton JM, Lou J, Xing L, Rubin BY (1999) Interferon-induced guanylate binding protein-1 (GBP-1) mediates an antiviral effect against vesicular stomatitis virus and encephalomyocarditis virus. Virology 256(1):8-14. https://doi. org/10.1006/viro.1999.9614

Antunes KH, Becker A, Franceschina C, de Freitas DD, Lape I, da Cunha MD, Leitão L, Rigo MM, Pinto LA, Stein RT et al (2019) Respiratory syncytial virus reduces STAT3 phosphorylation in human memory CD8 T cells stimulated with IL-21. Sci Rep 9(1):17766. https://doi.org/10.1038/s41598-019-54240-9

Apps R, Qi Y, Carlson JM, Chen H, Gao X, Thomas R, Yuki Y, Del Prete GQ, Goulder P, Brumme ZL et al (2013) Influence of HLA-C expression level on HIV control. Science 340(6128):8791. https://doi.org/10.1126/science.1232685

Arndt U, Wennemuth G, Barth P, Nain M, Al-Abed Y, Meinhardt A, Gemsa D, Bacher M (2002) Release of macrophage migration inhibitory factor and CXCL8/interleukin-8 from lung epithelial cells rendered necrotic by influenza A virus infection. J Virol 76(18):9298-9306. https://doi.org/10.1128/ jvi.76.18.9298-9306.2002

Asgari S, Schlapbach LJ, Anchisi S, Hammer C, Bartha I, Junier T, Mottet-Osman G, Posfay-Barbe KM, Longchamp D, Stocker $M$ et al (2017) Severe viral respiratory infections in children with IFIH1 loss-of-function mutations. Proc Natl Acad Sci USA 114(31):8342-8347. https://doi.org/10.1073/pnas.1704259114

Ayukawa H, Matsubara T, Kaneko M, Hasegawa M, Ichiyama T, Furukawa S (2004) Expression of CTLA - 4 (CD152) in peripheral blood T cells of children with influenza virus infection including encephalopathy in comparison with respiratory syncytial virus infection. Clin Exp Immunol 137(1):151-155. https://doi.org/1 0.1111/j.1365-2249.2004.02502.x

Baer A, Lundberg L, Swales D, Waybright N, Pinkham C, Dinman JD, Jacobs JL, Kehn-Hall K (2016) Venezuelan equine encephalitis virus induces apoptosis through the unfolded protein response activation of EGR1. J Virol 90(7):3558-3572. https://doi. org/10.1128/jvi.02827-15

Bertin J, Jalaguier P, Barat C, Roy MA, Tremblay MJ (2014) Exposure of human astrocytes to leukotriene $\mathrm{C} 4$ promotes a CX3CL1/ fractalkine-mediated transmigration of HIV-1-infected CD4+ $\mathrm{T}$ cells across an in vitro blood-brain barrier model. Virology 454-455:128-138. https://doi.org/10.1016/j.virol.2014.02.007

Bertrand P, Lay MK, Piedimonte G, Brockmann PE, Palavecino CE, Hernández J, León MA, Kalergis AM, Bueno SM (2015) Elevated IL-3 and IL-12p40 levels in the lower airway of infants with RSV-induced bronchiolitis correlate with recurrent wheezing. Cytokine 76(2):417-423. https://doi.org/10.1016/j. cyto.2015.07.017

Bongen E, Vallania F, Utz PJ, Khatri P (2018) KLRD1-expressing natural killer cells predict influenza susceptibility. Genome Med 10(1):45. https://doi.org/10.1186/s13073-018-0554-1

Bot A, Rodrigo E, Wolfe T, Bot S, Von Herrath MG (2003) Infection-triggered regulatory mechanisms override the role of STAT 4 in control of the immune response to influenza virus antigens. J Virol 77(10):5794-5800. https://doi.org/10.1128/ jvi.77.10.5794-5800.2003

Cai G, Nie X, Li L, Hu L, Wu B, Lin J, Jiang C, Wang H, Wang X, Shen Q (2013) B and T lymphocyte attenuator is highly expressed on intrahepatic T cells during chronic HBV infection and regulates their function. J Gastroenterol 48(12):1362-1372. https://doi.org/10.1007/s00535-013-0762-9

Calado M, Matoso P, Santos-Costa Q, Espirito-Santo M, Machado J, Rosado L, Antunes F, Mansinho K, Lopes MM, Maltez F et al (2010) Coreceptor usage by HIV-1 and HIV-2 primary isolates: the relevance of CCR8 chemokine receptor as an alternative coreceptor. Virology 408(2):174-182. https://doi.org/10.1016/j. virol.2010.09.020

Caspi R, Billington R, Ferrer L, Foerster H, Fulcher CA, Keseler IM, Kothari A, Krummenacker M, Latendresse M, Mueller LA et al (2016) The MetaCyc database of metabolic pathways 
and enzymes and the BioCyc collection of pathway/genome databases. Nucleic Acids Res 44(D1):D471-D480. https://doi. org/10.1093/nar/gkv1164

Castilow EM, Meyerholz DK, Varga SM (2008) IL-13 is required for eosinophil entry into the lung during respiratory syncytial virus vaccine-enhanced disease. J Immunol 180(4):2376-2384. https ://doi.org/10.4049/jimmunol.180.4.2376

Caufour P, Le Grand R, Chéret A, Neildez O, Thiébot H, Théodoro F, Boson B, Vaslin B, Venet A, Dormont D (2001) Longitudinal analysis of CD8(+) T-cell phenotype and IL-7, IL-15 and IL-16 mRNA expression in different tissues during primary simian immunodeficiency virus infection. Microbes Infect 3(3):181191. https://doi.org/10.1016/s1286-4579(01)01370-3

Chan KY, Ching JC, Xu MS, Cheung AN, Yip SP, Yam LY, Lai ST, Chu CM, Wong AT, Song YQ et al (2007) Association of ICAM3 genetic variant with severe acute respiratory syndrome. J Infect Dis 196(2):271-280. https://doi.org/10.1086/518892

Chan KY, Xu MS, Ching JC, So TM, Lai ST, Chu CM, Yam LY, Wong AT, Chung PH, Chan VS et al (2010) CD209 (DC-SIGN) $-336 \mathrm{~A}>\mathrm{G}$ promoter polymorphism and severe acute respiratory syndrome in Hong Kong Chinese. Hum Immunol 71(7):702-707. https://doi.org/10.1016/j.humimm.2010.03.006

Chen J, Bardes EE, Aronow BJ, Jegga AG (2009) ToppGene Suite for gene list enrichment analysis and candidate gene prioritization. Nucleic Acids Res 37(Web Server issue):W305-W311. https:// doi.org/10.1093/nar/gkp427

Chen C, Zhu X, Xu W, Yang F, Zhang G, Wu L, Zheng Y, Gao Z, Xie C, Peng L (2017a) IFNA2 p.Ala120Thr impairs the inhibitory activity of Interferon- $\alpha 2$ against the hepatitis B virus through altering its binding to the receptor. Antiviral Res 147:11-18. https://doi.org/10.1016/j.antiviral.2017.09.015

Chen X, Zhang Q, Bai J, Zhao Y, Wang X, Wang H, Jiang P (2017b) The nucleocapsid protein and nonstructural protein 10 of highly pathogenic porcine reproductive and respiratory syndrome virus enhance CD83 production via NF- $\kappa \mathrm{B}$ and Sp1 signaling pathways. J Virol 91(18):e00986. https://doi.org/10.1128/jvi.00986 $-17$

Chi CY, Chu CC, Liu JP, Lin CH, Ho MW, Lo WJ, Lin PC, Chen HJ, Chou CH, Feng JY et al (2013) Anti-IFN- $\gamma$ autoantibodies in adults with disseminated nontuberculous mycobacterial infections are associated with HLA-DRB $1 * 16: 02$ and HLADQB $1 * 05: 02$ and the reactivation of latent varicella-zoster virus infection. Blood 121(8):1357-1366. https://doi.org/10.1182/ blood-2012-08-452482

Chou CH, Shrestha S, Yang CD, Chang NW, Lin YL, Liao KW, Huang WC, Sun TH, Tu SJ, Lee WH et al (2018) miRTarBase update 2018: a resource for experimentally validated microRNA-target interactions. Nucleic Acids Res 46(D1):D296-D302. https://doi. org/10.1093/nar/gkx1067

Conti P, Ronconi G, Caraffa A, Gallenga CE, Ross R, Frydas I, Kritas SK (2020) Induction of pro-inflammatory cytokines (IL-1 and IL-6) and lung inflammation by Coronavirus-19 (COVI-19 or SARS-CoV-2): anti-inflammatory strategies. J Biol Regul Homeost Agents 34(2): 1

Corominas H, Castellví I, Domingo P, Casademont J (2020) Facing the SARS-CoV-2 (COVID-19) outbreak with IL-6R antagonists. Eur J Rheumatol. https://doi.org/10.5152/eurjrheum.2020.20061

Crabtree JN, He W, Guan W, Flage M, Miller MS, Peterson EJ (2016) Autoimmune Variant PTPN22 C1858T Is Associated With Impaired Responses to Influenza Vaccination. J Infect Dis 214(2):248-257. https://doi.org/10.1093/infdis/jiw126

Dahlquist KD, Salomonis N, Vranizan K, Lawlor SC, Conklin BR (2002) GenMAPP, a new tool for viewing and analyzing microarray data on biological pathways. Nat Genet 31(1):19-20. https ://doi.org/10.1038/ng0502-19
Dai E, Yu X, Zhang Y, Meng F, Wang S, Liu X, Liu D, Wang J, Li X, Jiang W (2014) EpimiR: a database of curated mutual regulation between miRNAs and epigenetic modifications. Database (Oxford). 2014:bau023. https://doi.org/10.1093/database/bau023

Dawson TC, Beck MA, Kuziel WA, Henderson F, Maeda N (2000) Contrasting effects of CCR5 and CCR2 deficiency in the pulmonary inflammatory response to influenza A virus. Am J Pathol 156(6):1951-1959. https://doi.org/10.1016/s0002 -9440(10)65068-7

Deng S, Yang C, Nie K, Fan S, Zhu M, Zhu J, Chen Y, Yuan J, Zhang J, Xu H et al (2019) Host cell protein PSMB10 interacts with viral NS3 protein and inhibits the growth of classical swine fever virus. Virology 537:74-83. https://doi.org/10.1016/j.virol 2019.05.017

Desai P, Tahiliani V, Abboud G, Stanfield J, Salek-Ardakani S (2018) Batf3-dependent dendritic cells promote optimal CD8 $\mathrm{T}$ cell responses against respiratory poxvirus infection. J Virol 92(16):e00495. https://doi.org/10.1128/jvi.00495-18

Dodd JS, Lum E, Goulding J, Muir R, Van Snick J, Openshaw PJ (2009) IL-9 regulates pathology during primary and memory responses to respiratory syncytial virus infection. J Immunol 183(11):7006-7013. https://doi.org/10.4049/jimmunol.0900085

Du N, Kwon H, Li P, West EE, Oh J, Liao W, Yu Z, Ren M, Leonard WJ (2014) EGR2 is critical for peripheral naïve T-cell differentiation and the T-cell response to influenza. Proc Natl Acad Sci USA 111(46):16484-16489. https://doi.org/10.1073/pnas.14172 15111

Durrant DM, Daniels BP, Klein RS (2014) IL-1R1 signaling regulates CXCL12-mediated $\mathrm{T}$ cell localization and fate within the central nervous system during West Nile Virus encephalitis. J Immunol 193(8):4095-4106. https://doi.org/10.4049/jimmunol.1401192

Earnest JT, Hantak MP, Li K, McCray PB Jr, Perlman S, Gallagher T (2017) The tetraspanin CD9 facilitates MERS-coronavirus entry by scaffolding host cell receptors and proteases. PLoS Pathog 13(7):e1006546. https://doi.org/10.1371/journal.ppat.1006546

El Saadany SA, Ziada DH, Farrag W, Hazaa S (2011) Fibrosis severity and mannan-binding lectin (MBL)/MBL-associated serine protease 1 (MASP-1) complex in $\mathrm{HCV}$-infected patients. Arab J Gastroenterol 12(2):68-73. https://doi.org/10.1016/j. ajg.2011.04.005

Ermers MJ, Janssen R, Onland-Moret NC, Hodemaekers HM, Rovers MM, Houben ML, Kimpen JL, Bont LJ (2011) IL10 family member genes IL19 and IL20 are associated with recurrent wheeze after respiratory syncytial virus bronchiolitis. Pediatr Res 70(5):518-523. https://doi.org/10.1203/pdr.0b013e31822f5863

Estrabaud E, Appourchaux K, Bieche I, Carrat F, Lapalus M, Lada O, Martinot-Peignoux M, Boyer N, Marcellin P, Vidaud M et al (2015) IFI35, mir-99a and HCV genotype to predict sustained virological response to pegylated-interferon plus ribavirin in chronic hepatitis C. PLoS ONE 10(4):e0121395. https://doi. org/10.1371/journal.pone.0121395

Estrada-Aguirre JA, Osuna-Ramírez I, Montes Prado, de Oca E, OchoaRamirez LA, Ramirez M, Magallon-Zazueta LG, Gonzalez-Beltran MS, Cazarez-Salazar SG, Rangel-Villalobos H, Velarde-Felix JS (2014) DEFB1 5'UTR polymorphisms modulate the risk of HIV-1 infection in Mexican women. Curr HIV Res 12(3):220 226. https://doi.org/10.2174/1570162x12666140708102722

Ezinne CC, Yoshimitsu M, White Y, Arima N (2014) HTLV-1 specific $\mathrm{CD} 8+\mathrm{T}$ cell function augmented by blockade of $2 \mathrm{~B} 4 / \mathrm{CD} 48$ interaction in HTLV-1 infection. PLoS ONE 9(2):e87631. https ://doi.org/10.1371/journal.pone.0087631

Fabregat A, Jupe S, Matthews L, Sidiropoulos K, Gillespie M, Garapati P, Haw R, Jassal B, Korninger F, May B et al (2018) The reactome pathway knowledgebase. Nucleic Acids Res 46(D1):D649D655. https://doi.org/10.1093/nar/gkx1132

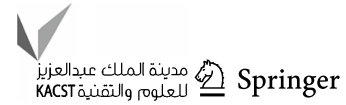


Fahrbach KM, Barry SM, Ayehunie S, Lamore S, Klausner M, Hope TJ (2007) Activated CD34-derived Langerhans cells mediate transinfection with human immunodeficiency virus. J Virol 81(13):6858-6868. https://doi.org/10.1128/jvi.02472-06

Fan Y, Xia J (2018) miRNet-functional analysis and visual exploration of mirna-target interactions in a network context. Methods Mol Biol 1819:215-233. https://doi. org/10.1007/978-1-4939-8618-7_10

Fan Y, Mok CK, Chan MC, Zhang Y, Nal B, Kien F, Bruzzone R, Sanyal S (2017) Cell cycle-independent role of cyclin D3 in host restriction of influenza virus infection. J Biol Chem 292(12):5070-5088. https://doi.org/10.1074/jbc.m117.776112

Fleming-Canepa X, Brusnyk C, Aldridge JR, Ross KL, Moon D, Wang D, Xia J, Barber MR, Webster RG, Magor KE (2011) Expression of duck CCL19 and CCL21 and CCR7 receptor in lymphoid and influenza-infected tissues. Mol Immunol 48(15-16):1950-1957. https://doi.org/10.1016/j.molimm.2011.05.025

Fossum E, Grødeland G, Terhorst D, Tveita AA, Vikse E, Mjaaland S, Henri S, Malissen B, Bogen B (2015) Vaccine molecules targeting Xcr1 on cross-presenting DCs induce protective CD8 + T-cell responses against influenza virus. Eur J Immunol 45(2):624-635. https://doi.org/10.1002/eji.201445080

Fox JM, Crabtree JM, Sage LK, Tompkins SM, Tripp RA (2015) Interferon lambda upregulates IDO1 expression in respiratory epithelial cells after influenza virus infection. J Interferon Cytokine Res 35(7):554-562. https://doi.org/10.1089/jir.2014.0052

Fu Y, Cheng Y, Wu Y (2020) Understanding SARS-CoV-2-mediated inflammatory responses: from mechanisms to potential therapeutic tools. Virol Sin. https://doi.org/10.1007/s12250-020-00207-4

Gaggar A, Shayakhmetov DM, Lieber A (2003) CD46 is a cellular receptor for group B adenoviruses. Nat Med 9(11):1408-1412. https://doi.org/10.1038/nm952

Gagné B, Tremblay N, Park AY, Baril M, Lamarre D (2017) Importin $\beta 1$ targeting by hepatitis $\mathrm{C}$ virus NS3/4A protein restricts IRF3 and NF- $\mathrm{kB}$ signaling of IFNB1 antiviral response. Traffic 18(6):362-377. https://doi.org/10.1111/tra.12480

García M, Cooper A, Shi W, Bornmann W, Carrion R, Kalman D, Nabel GJ (2012) Productive replication of Ebola virus is regulated by the c-Abl1 tyrosine kinase. Sci Transl Med 4(123):123ra24. https://doi.org/10.1126/scitranslmed.3003500

Gehlert T, Devergne O, Niedobitek G (2004) Epstein-Barr virus (EBV) infection and expression of the interleukin-12 family member EBV-induced gene 3 (EBI3) in chronic inflammatory bowel disease. J Med Virol 73(3):432-438. https://doi.org/10.1002/ jmv.20109

Girkin J, Hatchwell L, Foster P, Johnston SL, Bartlett N, Collison A, Mattes J (2015) CCL7 and IRF-7 Mediate Hallmark Inflammatory and IFN Responses following Rhinovirus 1B Infection. J Immunol 194(10):4924-4930. https://doi.org/10.4049/jimmu nol.1401362

Golden-Mason L, Burton JR Jr, Castelblanco N, Klarquist J, Benlloch S, Wang C, Rosen HR (2006) Loss of IL-7 receptor alphachain (CD127) expression in acute HCV infection associated with viral persistence. Hepatology 44(5):1098-1109. https://doi. org/10.1002/hep. 21365

Gorski SA, Hahn YS, Braciale TJ (2013) Group 2 innate lymphoid cell production of IL-5 is regulated by NKT cells during influenza virus infection. PLoS Pathog 9(9):e1003615. https://doi. org/10.1371/journal.ppat.1003615

Guévin C, Manna D, Bélanger C, Konan KV, Mak P, Labonté P (2010) Autophagy protein ATG5 interacts transiently with the hepatitis $\mathrm{C}$ virus RNA polymerase (NS5B) early during infection. Virology 405(1):1-7. https://doi.org/10.1016/j.virol.2010.05.032

Guntermann C, Dye J, Nye KE (1997) Human immunodeficiency virus infection abolishes CD4-dependent activation of ZAP-70 by inhibition of p561ck. J Acquir Immune Defic Syndr Hum Retrovirol 14(3):204-212. https://doi.org/10.1097/00042560-19970 3010-00002

Hansen MD, Johnsen IB, Stiberg KA, Sherstova T, Wakita T, Richard GM, Kandasamy RK, Meurs EF, Anthonsen MW (2017) Hepatitis $\mathrm{C}$ virus triggers Golgi fragmentation and autophagy through the immunity-related GTPase M. Proc Natl Acad Sci USA 114(17):E3462-E3471. https://doi.org/10.1073/pnas.16166 83114

Harcourt JL, Brown MP, Anderson LJ, Tripp RA (2003) CD40 ligand (CD154) improves the durability of respiratory syncytial virus DNA vaccination in BALB/c mice. Vaccine 21(21-22):29642979. https://doi.org/10.1016/s0264-410x(03)00119-1

Hirai A, Ohtsuka N, Ikeda T, Taniguchi R, Blau D, Nakagaki K, Miura HS, Ami Y, Yamada YK, Itohara S et al (2010) Role of mouse hepatitis virus (MHV) receptor murine CEACAM1 in the resistance of mice to MHV infection: studies of mice with chimeric mCEACAM1a and mCEACAM1b. J Virol 84(13):6654-6666. https://doi.org/10.1128/jvi.02680-09

Hiramoto T, Li LB, Funk SE, Hirata RK, Russell DW (2018) Nuclease-free adeno-associated virus-mediated $\mathrm{I} 12 \mathrm{rg}$ gene editing in X-SCID mice. Mol Ther 26(5):1255-1265. https://doi. org/10.1016/j.ymthe.2018.02.028

Hoffmann M, Kleine-Weber H, Schroeder S, Krüger N, Herrler T, Erichsen S, Schiergens TS, Herrler G, Wu NH, Nitsche A et al (2020) SARS-CoV-2 cell entry depends on ACE2 and TMPRSS2 and is blocked by a clinically proven protease inhibitor. Cell 181(2):271-280. https://doi.org/10.1016/j.cell.2020.02.052 e8

Hou S, Doherty PC (1993) Partitioning of responder CD8 + T cells in lymph node and lung of mice with Sendai virus pneumonia by LECAM-1 and CD45RB phenotype. J Immunol 150(12):5494-5500

Hsu EC, Hsi B, Hirota-Tsuchihara M, Ruland J, Iorio C, Sarangi F, Diao J, Migliaccio G, Tyrrell DL, Kneteman N et al (2003) Modified apoptotic molecule (BID) reduces hepatitis $\mathrm{C}$ virus infection in mice with chimeric human livers. Nat Biotechnol 21(5):519-525. https://doi.org/10.1038/nbt817

Hu M, Li HM, Bogoyevitch MA, Jans DA (2017) Mitochondrial protein $\mathrm{p} 32 / \mathrm{HAPB} 1 / \mathrm{gC} 1 \mathrm{qR} / \mathrm{C} 1 \mathrm{qbp}$ is required for efficient respiratory syncytial virus production. Biochem Biophys Res Commun 489(4):460-465. https://doi.org/10.1016/j.bbrc.2017.05.171

Huang Z, Shi J, Gao Y, Cui C, Zhang S, Li J, Zhou Y, Cui Q (2019) HMDD v.30: a database for experimentally supported human microRNA-disease associations. Nucleic Acids Res 47(D1):D1013-D1017. https://doi.org/10.1093/nar/gky1010z

Imakiire K, Uto H, Sato Y, Sasaki F, Mawatari S, Ido A, Shimoda K, Hayashi K, Stuver SO, Ito Y et al (2012) Difference in serum complement component $\mathrm{C} 4 \mathrm{a}$ levels between hepatitis $\mathrm{C}$ virus carriers with persistently normal alanine aminotransferase levels or chronic hepatitis C. Mol Med Rep 6(2):259-264. https://doi. org/10.3892/mmr.2012.924

Jewison T, Su Y, Disfany FM, Liang Y, Knox C, Maciejewski A, Poelzer J, Huynh J, Zhou Y, Arndt D et al (2014) SMPDB 2.0: big improvements to the Small Molecule Pathway Database. Nucleic Acids Res 42(Database issue):D478-D484. https://doi. org/10.1093/nar/gkt1067

Jiang Q, Wang Y, Hao Y, Juan L, Teng M, Zhang X, Li M, Wang G, Liu $Y$ (2009) miR2Disease: a manually curated database for microRNA deregulation in human disease. Nucleic Acids Res 37(Database issue):D98-D104. https://doi.org/10.1093/nar/gkn714

Kanehisa M, Sato Y, Furumichi M, Morishima K, Tanabe M (2019) New approach for understanding genome variations in KEGG. Nucleic Acids Res 47(D1):D590-D595. https://doi.org/10.1093/ nar/gky962 
Kenney SP, Meng XJ (2015) An SH3 binding motif within the nucleocapsid protein of porcine reproductive and respiratory syndrome virus interacts with the host cellular signaling proteins STAMI, TXK, Fyn, Hck, and cortactin. Virus Res 204:31-39. https://doi. org/10.1016/j.virusres.2015.04.004

Khan A, Fornes O, Stigliani A, Gheorghe M, Castro-Mondragon JA, van der Lee R, Bessy A, Chèneby J, Kulkarni SR, Tan G et al (2018) JASPAR 2018: update of the open-access database of transcription factor binding profiles and its web framework. Nucleic Acids Res 46(D1):D260-D266. https://doi.org/10.1093/ nar/gkx 1126

Kim H, Meyer K, Di Bisceglie AM, Ray R (2013) Hepatitis C virus suppresses $\mathrm{C} 9$ complement synthesis and impairs membrane attack complex function. J Virol 87(10):5858-5867. https://doi. org/10.1128/jvi.00174-13

Kolesnikov N, Hastings E, Keays M, Melnichuk O, Tang YA, Williams E, Dylag M, Kurbatova N, Brandizi M, Burdett T, Megy K (2015) Arrayexpress update-simplifying data submissions. Nucleic Acids Res 43(Database issue):D1113-D1116. https:// doi.org/10.1093/nar/gku1057

Koraka P, Murgue B, Deparis X, van Gorp EC, Setiati TE, Osterhaus AD, Groen J (2004) Elevation of soluble VCAM-1 plasma levels in children with acute dengue virus infection of varying severity. J Med Virol 72(3):445-450. https://doi.org/10.1002/jmv.20007

Kumar P, Thakar MS, Ouyang W, Malarkannan S (2013) IL-22 from conventional NK cells is epithelial regenerative and inflammation protective during influenza infection. Mucosal Immunol 6(1):69-82. https://doi.org/10.1038/mi.2012.49

Lacher MD, Shiina M, Chang P, Keller D, Tiirikainen MI, Korn WM (2011) ZEB1 limits adenoviral infectability by transcriptionally repressing the coxsackie virus and adenovirus receptor. Mol Cancer 10:91. https://doi.org/10.1186/1476-4598-10-91

Lambert ND, Haralambieva IH, Kennedy RB, Ovsyannikova IG, Pankratz VS, Poland GA (2015) Polymorphisms in HLA-DPB1 are associated with differences in rubella virus-specific humoral immunity after vaccination. J Infect Dis 211(6):898-905. https ://doi.org/10.1093/infdis/jiu553

Le Noury DA, Mosebi S, Papathanasopoulos MA, Hewer R (2015) Functional roles of HIV-1 Vpu and CD74: details and implications of the Vpu-CD74 interaction. Cell Immunol 298(1-2):2532. https://doi.org/10.1016/j.cellimm.2015.08.005

Letko M, Miazgowicz K, McMinn R, Seifert SN, Sola I, Enjuanes L, Carmody A, Van Doremalen N, Munster V (2018) Adaptive evolution of MERS-CoV to Species Variation in DPP4. Cell Rep 24(7):1730-1737. https://doi.org/10.1016/j.celrep.2018.07.045

Lewis SE (2017) The vision and challenges of the gene ontology. Methods Mol Biol 1446:291-302. https://doi. org/10.1007/978-1-4939-3743-1_21

Li JH, Liu S, Zhou H, Qu LH, Yang JH (2014) starBase v20: decoding miRNA-ceRNA, miRNA-ncRNA and protein-RNA interaction networks from large-scale CLIP-Seq data. Nucleic Acids Res 42(Databse Issue):D92-D97. https://doi.org/10.1093/nar/gkt12 48

Li C, Kuang WD, Qu D, Wang JH (2016a) Toll-interacting protein inhibits HIV-1 infection and regulates viral latency. Biochem Biophys Res Commun 475(2):161-168. https://doi.org/10.1016/j. bbrc.2016.05.065

Li Y, Johnson JB, Parks GD (2016b) Parainfluenza virus 5 upregulates CD55 expression to produce virions with enhanced resistance to complement-mediated neutralization. Virology 497:305-313. https://doi.org/10.1016/j.virol.2016.07.030

Li YK, Peng S, Li LQ, Wang Q, Ping W, Zhang N, Fu XN (2020) Clinical and transmission characteristics of Covid-19-a retrospective study of 25 cases from a single thoracic surgery department. Curr Med Sci 40(2):295-300. https://doi.org/10.1007/s1159 6-020-2176-2
Lin SJ, Lo M, Kuo RL, Shih SR, Ojcius DM, Lu J, Lee CK, Chen HC, Lin MY, Leu CM et al (2014) The pathological effects of CCR2 + inflammatory monocytes are amplified by an IFNAR1triggered chemokine feedback loop in highly pathogenic influenza infection. J Biomed Sci 21(1):99. https://doi.org/10.1186/ s12929-014-0099-6

Liu Y, Nonnemacher MR, Wigdahl B (2009) CCAAT/enhancer-binding proteins and the pathogenesis of retrovirus infection. Future Microbiol 4(3):299-321. https://doi.org/10.2217/fmb.09.4

Liu X, Wang S, Meng F, Wang J, Zhang Y, Dai E, Yu X, Li X, Jiang W (2013) SM2miR: a database of the experimentally validated small molecules' effects on microRNA expression. Bioinformatics 29(3):409-411. https://doi.org/10.1093/bioinformatics/bts698

Liu B, Zhang X, Deng W, Liu J, Li H, Wen M, Bao L, Qu J, Liu Y, Li $F$ et al (2014) Severe influenza A(H1N1)pdm09 infection induces thymic atrophy through activating innate CD8(+)CD44(hi) T cells by upregulating IFN- $\gamma$. Cell Death Dis 5(10):e1440. https ://doi.org/10.1038/cddis.2014.323

Liu X, Bi J, Zhao Q, Li M, Zuo Q, Wang X, Lan R, Li X, Yang G, Liu J et al (2019) Overexpression of RACK1 enhanced the replication of porcine reproductive and respiratory syndrome virus in Marc145 cells and promoted the NF- $\mathrm{KB}$ activation via upregulating the expression and phosphorylation of TRAF2. Gene 709:75-83. https://doi.org/10.1016/j.gene.2019.05.046

Loebbermann J, Schnoeller C, Thornton H, Durant L, Sweeney NP, Schuijs M, O'Garra A, Johansson C, Openshaw PJ (2012a) IL-10 regulates viral lung immunopathology during acute respiratory syncytial virus infection in mice. PLoS ONE 7(2):e32371. https ://doi.org/10.1371/journal.pone.0032371

Loebbermann J, Thornton H, Durant L, Sparwasser T, Webster KE, Sprent J, Culley FJ, Johansson C, Openshaw PJ (2012b) Regulatory $\mathrm{T}$ cells expressing granzyme B play a critical role in controlling lung inflammation during acute viral infection. Mucosal Immunol 5(2):161-172. https://doi.org/10.1038/mi.2011.62

Lu JW, Chang JG, Yeh KT, Chen RM, Tsai JJ, Hu RM (2011) Overexpression of Thy1/CD90 in human hepatocellular carcinoma is associated with HBV infection and poor prognosis. Acta Histochem 113(8):833-838. https://doi.org/10.1016/j.acthi s.2011.01.001

Lumsden JM, Roberts JM, Harris NL, Peach RJ, Ronchese F (2000) Differential requirement for CD80 and CD80/CD86-dependent costimulation in the lung immune response to an influenza virus infection. J Immunol 164(1):79-85. https://doi.org/10.4049/ jimmunol.164.1.79

Ma DY, Suthar MS, Kasahara S, Gale M Jr, Clark EA (2013) CD22 is required for protection against West Nile virus Infection. J Virol 87(6):3361-3375. https://doi.org/10.1128/jvi.02368-12

Madurai Elavarasan R, Pugazhendhi R (2020) Restructured society and environment: a review on potential technological strategies to control the COVID-19 pandemic. Sci Total Environ 725:138858. https://doi.org/10.1016/j.scitotenv.2020.138858

Maestri A, Sortica VA, Ferreira DL, de Almeida Ferreira J, Amador MA, de Mello WA, Santos SE, Sousa RC (2016) The His131Arg substitution in the FCGR2A gene (rs1801274) is not associated with the severity of influenza A(H1N1)pdm09 infection. BMC Res Notes 9:296. https://doi.org/10.1186/ s13104-016-2096-1

Malhotra P, Haslett P, Sherry B, Shepp DH, Barber P, Abshier J, Roy U, Schmidtmayerova H (2019) Increased plasma levels of the TH2 chemokine CCL18 associated with low CD4 + T cell counts in HIV-1-infected patients with a suppressed viral load. Sci Rep 9(1):5963. https://doi.org/10.1038/s41598-01941588-1

Martin MP, Qi Y, Gao X, Yamada E, Martin JN, Pereyra F, Colombo S, Brown EE, Shupert WL, Phair J et al (2007) Innate partnership

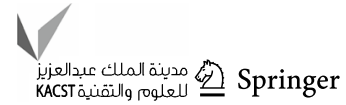


of HLA-B and KIR3DL1 subtypes against HIV-1. Nat Genet 39(6):733-740. https://doi.org/10.1038/ng2035

Mi H, Huang X, Muruganujan A, Tang H, Mills C, Kang D, Thomas PD (2017) PANTHER version 11: expanded annotation data from Gene Ontology and Reactome pathways, and data analysis tool enhancements. Nucleic Acids Res 45(D1):D183-D189. https ://doi.org/10.1093/nar/gkw1138

Miller AL, Gerard C, Schaller M, Gruber AD, Humbles AA, Lukacs NW (2006) Deletion of CCR1 attenuates pathophysiologic responses during respiratory syncytial virus infection. J Immunol 176(4):2562-2567. https://doi.org/10.4049/jimmunol.176.4.2562

Miot C, Beaumont E, Duluc D, Le Guillou-Guillemette H, Preisser L, Garo E, Blanchard S, Fouchard IH, Créminon C, Lamourette P et al (2015) IL-26 is overexpressed in chronically HCV-infected patients and enhances TRAIL-mediated cytotoxicity and interferon production by human NK cells. Gut 64(9):1466-1475. https ://doi.org/10.1136/gutjnl-2013-306604

Mizutani T, Fukushi S, Murakami M, Hirano T, Saijo M, Kurane I, Morikawa S (2004) Tyrosine dephosphorylation of STAT3 in SARS coronavirus-infected Vero E6 cells. FEBS Lett 577(12):187-192. https://doi.org/10.1016/j.febslet.2004.10.005

Morales-García G, Falfán-Valencia R, García-Ramírez RA, Camarena Á, Ramirez-Venegas A, Castillejos-López M, Pérez-Rodríguez M, González-Bonilla C, Grajales-Muñíz C, Borja-Aburto V et al (2012) Pandemic influenza A/H1N1 virus infection and TNF, LTA, IL1B, IL6, IL8, and CCL polymorphisms in Mexican population: a case-control study. BMC Infect Dis 12:299. https://doi. org/10.1186/1471-2334-12-299

Mueller T, Mas-Marques A, Sarrazin C, Wiese M, Halangk J, Witt H, Ahlenstiel G, Spengler U, Goebel U, Wiedenmann B et al (2004) Influence of interleukin 12B (IL12B) polymorphisms on spontaneous and treatment-induced recovery from hepatitis $\mathrm{C}$ virus infection. J Hepatol 41(4):652-658. https://doi.org/10.1016/j. jhep.2004.06.021

Mukherjee S, Rasky AJ, Lundy PA, Kittan NA, Kunkel SL, Maillard IP, Kowalski PE, Kousis PC, Guidos CJ, Lukacs NW (2014) STAT5-induced lunatic fringe during Th2 development alters delta-like 4-mediated Th2 cytokine production in respiratory syncytial virus-exacerbated airway allergic disease. J Immunol 192(3):996-1003. https://doi.org/10.4049/jimmunol.1301991

Nagarkar DR, Wang Q, Shim J, Zhao Y, Tsai WC, Lukacs NW, Sajjan U, Hershenson MB (2009) CXCR2 is required for neutrophilic airway inflammation and hyperresponsiveness in a mouse model of human rhinovirus infection. J Immunol 183(10):6698-6707. https://doi.org/10.4049/jimmunol.0900298

Nakayama T, Fujisawa R, Izawa D, Hieshima K, Takada K, Yoshie O (2002) Human B cells immortalized with Epstein-Barr virus upregulate CCR6 and CCR10 and downregulate CXCR4 and CXCR5. J Virol 76(6):3072-3077. https://doi.org/10.1128/ jvi.76.6.3072-3077.2002

Nasi M, Riva A, Borghi V, D’Amico R, Del Giovane C, Casoli C, Galli M, Vicenzi E, Gibellini L, De Biasi S et al (2013) Novel genetic association of TNF- $\alpha-238$ and PDCD1-7209 polymorphisms with long-term non-progressive HIV-1 infection. Int J Infect Dis 17(10):e845-e850. https://doi.org/10.1016/j.ijid.2013.01.003

Nguyen TP, Liu WC, Jordán F (2011) Inferring pleiotropy by network analysis: linked diseases in the human PPI network. BMC Syst Biol 5:179. https://doi.org/10.1186/1752-0509-5-179

Noma T, Mori A, Yoshizawa I (1996) Induction of allergen-specific IL-2 responsiveness of lymphocytes after respiratory syncytial virus infection and prediction of onset of recurrent wheezing and bronchial asthma. J Allergy Clin Immunol 98(4):816-826. https://doi.org/10.1016/s0091-6749(96)70131-8

O’Connell P, Pepelyayeva Y, Blake MK, Hyslop S, Crawford RB, Rizzo MD, Pereira-Hicks C, Godbehere S, Dale L, Gulick P et al (2019) SLAMF7 is a critical negative regulator of IFN$\alpha$-mediated CXCL10 production in chronic HIV infection. J Immunol 202(1):228-238. https://doi.org/10.4049/jimmu nol.1800847

Ohno S, Kohyama S, Taneichi M, Moriya O, Hayashi H, Oda H, Mori M, Kobayashi A, Akatsuka T, Uchida T et al (2009) Synthetic peptides coupled to the surface of liposomes effectively induce SARS coronavirus-specific cytotoxic T lymphocytes and viral clearance in HLA-A*0201 transgenic mice. Vaccine 27(29):3912-3920. https://doi.org/10.1016/j.vacci ne.2009.04.001

Patel D, Nan Y, Shen M, Ritthipichai K, Zhu X, Zhang YJ (2010) Porcine reproductive and respiratory syndrome virus inhibits type I interferon signaling by blocking STAT1/STAT2 nuclear translocation. J Virol 84(21):11045-11055. https://doi.org/10.1128/ jvi.00655-10

Petri V, Jayaraman P, Tutaj M, Hayman GT, Smith JR, De Pons J, Laulederkind SJ, Lowry TF, Nigam R, Wang SJ (2014) The pathway ontology - updates and applications. J Biomed Semant 5(1):7. https://doi.org/10.1186/2041-1480-5-7

Pillai PS, Molony RD, Martinod K, Dong H, Pang IK, Tal MC, Solis AG, Bielecki P, Mohanty S, Trentalange M et al (2016) $\mathrm{Mx} 1$ reveals innate pathways to antiviral resistance and lethal influenza disease. Science 352(6284):463-466. https://doi. org/10.1126/science.aaf3926

Pineda-Tenor D, Berenguer J, Jiménez-Sousa MA, Guzmán-Fulgencio M, Aldámiz-Echevarria T, Carrero A, García-Álvarez M, Diez C, Tejerina F, Briz V et al (2014) CXCL9, CXCL10 and CXCL11 polymorphisms are associated with sustained virologic response in HIV/HCV-coinfected patients. J Clin Virol 61(3):423-429. https://doi.org/10.1016/j.jcv.2014.08.020

Posada R, Pettoello-Mantovani M, Sieweke M, Graf T, Goldstein H (2000) Suppression of HIV type 1 replication by a dominant-negative Ets-1 mutant. AIDS Res Hum Retroviruses 16(18):19811989. https://doi.org/10.1089/088922200750054710

Pothlichet J, Meunier I, Davis BK, Ting JP, Skamene E, von Messling V, Vidal SM (2013) Type I IFN triggers RIG-I/TLR3/NLRP3dependent inflammasome activation in influenza A virus infected cells. PLoS Pathog 9(4):e1003256. https://doi.org/10.1371/journ al.ppat.1003256

Przulj N, Wigle DA, Jurisica I (2004) Functional topology in a network of protein interactions. Bioinformatics 20(3):340-348. https:// doi.org/10.1093/bioinformatics/btg415

Puthothu B, Krueger M, Forster J, Heinzmann A (2006) Association between severe respiratory syncytial virus infection and IL13/IL4 haplotypes. J Infect Dis 193(3):438-441. https://doi. org/10.1086/499316

Raziorrouh B, Schraut W, Gerlach T, Nowack D, Grüner NH, Ulsenheimer A, Zachoval R, Wächtler M, Spannagl M, Haas J et al (2010) The immunoregulatory role of CD244 in chronic hepatitis $\mathrm{B}$ infection and its inhibitory potential on virus-specific CD8 + T-cell function. Hepatology 52(6):1934-1947. https://doi. org/10.1002/hep.23936

Rice TA, Brenner TA, Percopo CM, Ma M, Keicher JD, Domachowske JB, Rosenberg HF (2016) Signaling via pattern recognition receptors NOD2 and TLR2 contributes to immunomodulatory control of lethal pneumovirus infection. Antiviral Res 132:131140. https://doi.org/10.1016/j.antiviral.2016.06.002

Ritchie ME, Phipson B, Wu DI, Hu Y, Law CW, Shi W, Smyth GK (2015) limma powers differential expression analyses for RNAsequencing and microarray studies. Nucleic Acids Res 43(7):e47. https://doi.org/10.1093/nar/gkv007

Robin X, Turck N, Hainard A, Tiberti N, Lisacek F, Sanchez JC, Müller M (2011) pROC: an open-source package for R and S + to 
analyze and compare ROC curves. BMC Bioinformatics 12:77. https://doi.org/10.1186/1471-2105-12-77

Rodriguez R, Fournier B, Cordeiro DJ, Winter S, Izawa K, Martin E, Boutboul D, Lenoir C, Fraitag S, Kracker S et al (2019) Concomitant PIK3CD and TNFRSF9 deficiencies cause chronic active Epstein-Barr virus infection of T cells. J Exp Med 216(12):28002818. https://doi.org/10.1084/jem.20190678

Romporn S, Hirankarn N, Tangkijvanich P, Kimkong I (2013) Association of IFNAR2 and IL10RB genes in chronic hepatitis B virus infection. Tissue Antigens 82(1):21-25. https://doi.org/10.1111/ $\tan .12133$

Rosenberger CM, Podyminogin RL, Diercks AH, Treuting PM, Peschon JJ, Rodriguez D, Gundapuneni M, Weiss MJ, Aderem A (2017) miR-144 attenuates the host response to influenza virus by targeting the TRAF6-IRF7 signaling axis. PLoS Pathog 13(4):e1006305. https://doi.org/10.1371/journal.ppat.1006305

Ruepp A, Kowarsch A, Schmidl D, Buggenthin F, Brauner B, Dunger I, Fobo G, Frishman G, Montrone C, Theis FJ (2010) PhenomiR: a knowledgebase for microRNA expression in diseases and biological processes. Genome Biol 11(1):R6. https://doi.org/10.1186/ gb-2010-11-1-r6

Rukov JL, Wilentzik R, Jaffe I, Vinther J, Shomron N (2014) Pharmaco-miR: linking microRNAs and drug effects. Brief Bioinform 15(4):648-659. https://doi.org/10.1093/bib/bbs082

Sacchi A, Cappelli G, Cairo C, Martino A, Sanarico N, D'Offizi G, Pupillo LP, Chenal H, De Libero G, Colizzi V et al (2007) Differentiation of monocytes into CD1a- dendritic cells correlates with disease progression in HIV-infected patients. J Acquir Immune Defic Syndr 46(5):519-528. https://doi.org/10.1097/qai.0b013 e31815b1278

Sainz B Jr, Mossel EC, Peters CJ, Garry RF (2004) Interferon-beta and interferon-gamma synergistically inhibit the replication of severe acute respiratory syndrome-associated coronavirus (SARS-CoV). Virology 329(1):11-17. https://doi.org/10.1016/j. virol.2004.08.011

Salimi V, Ramezani A, Mirzaei H, Tahamtan A, Faghihloo E, Rezaei F, Naseri M, Bont L, Mokhtari-Azad T, Tavakoli-Yaraki M (2017) Evaluation of the expression level of 12/15 lipoxygenase and the related inflammatory factors (CCL5, CCL3) in respiratory syncytial virus infection in mice model. Microb Pathog 109:209-213. https://doi.org/10.1016/j.micpath.2017.05.045

Sanders SP, Siekierski ES, Richards SM, Porter JD, Imani F, Proud D (2001) Rhinovirus infection induces expression of type 2 nitric oxide synthase in human respiratory epithelial cells in vitro and in vivo. J Allergy Clin Immunol 107(2):235-243. https://doi. org/10.1067/mai.2001.112028

Sanfilippo C, Cambria D, Longo A, Palumbo M, Avola R, Pinzone M, Nunnari G, Condorelli F, Musumeci G, Imbesi R (2017) SERPING1 mRNA overexpression in monocytes from HIV + patients. Inflamm Res 66(12):1107-1116. https://doi.org/10.1007/s0001 1-017-1091-x

Schaefer CF, Anthony K, Krupa S, Buchoff J, Day M, Hannay T, Buetow KH (2009) PID: the pathway interaction database. Nucleic Acids Res 37(1):D674-D679. https://doi.org/10.1093/nar/gkn65 3

Shafique M, Wilschut J, de Haan A (2012) Induction of mucosal and systemic immunity against respiratory syncytial virus by inactivated virus supplemented with TLR9 and NOD2 ligands. Vaccine 30(3):597-606. https://doi.org/10.1016/j.vaccine.2011.11.054

Shannon P, Markiel A, Ozier O, Baliga NS, Wang JT, Ramage D, Amin N, Schwikowski B, Ideker T (2003) Cytoscape: a software environment for integrated models of biomolecular interaction networks. Genome Res 13(11):2498-2504. https://doi.org/10.1101/ gr.1239303
Sharma M, Bhattacharyya S, Sharma KB, Chauhan S, Asthana S, Abdin MZ, Vrati S, Kalia M (2017) Japanese encephalitis virus activates autophagy through XBP1 and ATF6 ER stress sensors in neuronal cells. J Gen Virol 98(5):1027-1039. https://doi. org/10.1099/jgv.0.000792

Shi Z, Zhang B (2011) Fast network centrality analysis using GPUs. BMC Bioinformatics 12:149. https://doi. org/10.1186/1471-2105-12-149

Shi L, Ramaswamy M, Manzel LJ, Look DC (2007) Inhibition of Jak1dependent signal transduction in airway epithelial cells infected with adenovirus. Am J Respir Cell Mol Biol 37(6):720-728. https://doi.org/10.1165/rcmb.2007-0158oc

Shi T, He Y, Sun W, Wu Y, Li L, Jie Z, Su X (2017) Respiratory Syncytial virus infection compromises asthma tolerance by recruiting interleukin-17A-producing cells via CCR6-CCL20 signaling. Mol Immunol 88:45-57. https://doi.org/10.1016/j.molim m.2017.05.017

Sidenius N, Sier CF, Ullum H, Pedersen BK, Lepri AC, Blasi F, EugenOlsen J (2000) Serum level of soluble urokinase-type plasminogen activator receptor is a strong and independent predictor of survival in human immunodeficiency virus infection. Blood 96(13):4091-4095

Siu KL, Kok KH, Ng MH, Poon VK, Yuen KY, Zheng BJ, Jin DY (2009) Severe acute respiratory syndrome coronavirus M protein inhibits type I interferon production by impeding the formation of TRAF3.TANK.TBK1/IKKepsilon complex. J Biol Chem 284(24):16202-16209. https://doi.org/10.1074/jbc.m109.008227

Soroosh P, Doherty TA, So T, Mehta AK, Khorram N, Norris PS, Scheu S, Pfeffer K, Ware C, Croft M (2014) Interactions between herpesvirus entry mediator (TNFRSF14) and latency-associated transcript during herpes simplex virus 1 latency. J Virol 88(4):1961-1971. https://doi.org/10.1128/jvi.02467-13

Spector SA, Singh KK, Gupta S, Cystique LA, Jin H, Letendre S, Schrier R, Wu Z, Hong KX, Yu X et al (2010) APOE epsilon4 and MBL-2 O/O genotypes are associated with neurocognitive impairment in HIV-infected plasma donors. AIDS 24(10):14711479. https://doi.org/10.1097/qad.0b013e328339e25c

Srinivasa BT, Restori KH, Shan J, Cyr L, Xing L, Lee S, Ward BJ, Fixman ED (2017) STAT6 inhibitory peptide given during RSV infection of neonatal mice reduces exacerbated airway responses upon adult reinfection. J Leukoc Biol 101(2):519-529. https:// doi.org/10.1189/jlb.4a0215-062rr

Subramanian A, Tamayo P, Mootha VK, Mukherjee S, Ebert BL, Gillette MA, Paulovich A, Pomeroy SL, Golub TR, Lander ES (2005) Gene set enrichment analysis: a knowledge-based approach for interpreting genome-wide expression profiles. Proc Natl Acad Sci USA 102(43):15545-15550. https://doi. org/10.1073/pnas.0506580102

Sungnak W, Huang N, Bécavin C, Berg M, Queen R, Litvinukova M, Talavera-López C, Maatz H, Reichart D, Sampaziotis F et al (2020) SARS-CoV-2 entry factors are highly expressed in nasal epithelial cells together with innate immune genes. Nat Med. https://doi.org/10.1038/s41591-020-0868-6

Suryadevara M, Bonville CA, Rosenberg HF, Domachowske JB (2013) Local production of CCL3, CCL11, and IFN- $\gamma$ correlates with disease severity in murine parainfluenza virus infection. Virol J 10:357. https://doi.org/10.1186/1743-422x-10-357

Swaminathan S, Dai L, Lane HC, Imamichi T (2013) Evaluating the potential of IL-27 as a novel therapeutic agent in HIV-1 infection. Cytokine Growth Factor Rev 24(6):571-577. https://doi. org/10.1016/j.cytogfr.2013.07.001

Szklarczyk D, Gable AL, Lyon D, Junge A, Wyder S, HuertaCepas J, Simonovic M, Doncheva NT, Morris JH, Bork P et al (2019) STRING v11: protein-protein association networks with increased coverage, supporting functional discovery

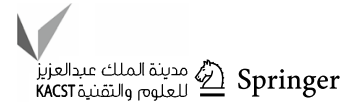


in genome-wide experimental datasets. Nucleic Acids Res 47(D1):D607-D613. https://doi.org/10.1093/nar/gky1131

Takahashi T, Takaguchi M, Kawakami T, Suzuki T (2013) Sulfatide regulates caspase-3-independent apoptosis of influenza A virus through viral PB1-F2 protein. PLoS ONE 8(4):e61092. https:// doi.org/10.1371/journal.pone.0061092

Terry RL, Deffrasnes C, Getts DR, Minten C, Van Vreden C, Ashhurst TM, Getts MT, Xie RD, Campbell IL, King NJ (2015) Defective inflammatory monocyte development in IRF8-deficient mice abrogates migration to the West Nile virus-infected brain. J Innate Immun 7(1):102-112. https://doi.org/10.1159/000365972

Tian X, Zhang A, Qiu C, Wang W, Yang Y, Qiu C, Liu A, Zhu L, Yuan S, Hu H et al (2015) The upregulation of LAG-3 on T cells defines a subpopulation with functional exhaustion and correlates with disease progression in HIV-infected subjects. J Immunol 194(8):3873-3882. https://doi.org/10.4049/jimmunol.1402176

Tian J, Jiao X, Wang X, Geng J, Wang R, Liu N, Gao X, Griffin N, Shan $F$ (2018) Novel effect of methionine enkephalin against influenza A virus infection through inhibiting TLR7-MyD88TRAF6-NF- $\mathrm{B}$ p65 signaling pathway. Int Immunopharmacol 55:38-48. https://doi.org/10.1016/j.intimp.2017.12.001

Tibbs C, Donaldson P, Underhill J, Thomson L, Manabe K, Williams R (1996) Evidence that the HLA DQA $1 * 03$ allele confers protection from chronic HCV-infection in Northern European Caucasoids. Hepatology 24(6):1342-1345. https://doi.org/10.1053/ jhep.1996.v24.pm0008938158

Tochikura TS, Xiao S, Ego T, Sagara J, Kawai A (2003) Further characterization of a CD99-related 21-kDa transmembrane protein (VAP21) expressed in Syrian hamster cells and its possible involvement in vesicular stomatitis virus production. Microbiol Immunol 47(10):745-757. https://doi. org/10.1111/j.1348-0421.2003.tb03444.x

Treon SP, Castillo J, Skarbnik AP, Soumerai JD, Ghobrial IM, Guerrera ML, Meid KE, Yang G (2020) The BTK-inhibitor ibrutinib may protect against pulmonary injury in COVID-19 infected patients. Blood. https://doi.org/10.1182/blood.2020006288

Tucci FA, Broering R, Johansson P, Schlaak JF, Küppers R (2013) B cells in chronically hepatitis $\mathrm{C}$ virus-infected individuals lack a virus-induced mutation signature in the TP53, CTNNB1, and BCL6 genes. J Virol 87(5):2956-2962. https://doi.org/10.1128/ jvi.03081-12

Uematsu T, Iizasa E, Kobayashi N, Yoshida H, Hara H (2015) Loss of CARD9-mediated innate activation attenuates severe influenza pneumonia without compromising host viral immunity. Sci Rep 5:17577. https://doi.org/10.1038/srep17577

Vandenbroeck K, Alloza I, Swaminathan B, Antigüedad A, Otaegui D, Olascoaga J, Barcina MG, De Las Heras V, Bartolomé M, Fernández-Arquero M et al (2011) Validation of IRF5 as multiple sclerosis risk gene: putative role in interferon beta therapy and human herpes virus-6 infection. Genes Immun 12(1):40-45. https://doi.org/10.1038/gene.2010.46

Vlachos IS, Paraskevopoulou MD, Karagkouni D, Georgakilas G, Vergoulis T, Kanellos I, Anastasopoulos IL, Maniou S, Karathanou K, Kalfakakou D et al (2015) DIANA-TarBase v7.0: indexing more than half a million experimentally supported miRNA:mRNA interactions. Nucleic Acids Res 43(1):D153D159. https://doi.org/10.1093/nar/gku1215

Wang SZ, Smith PK, Lovejoy M, Bowden JJ, Alpers JH, Forsyth KD (1998) Shedding of L-selectin and PECAM-1 and upregulation of Mac-1 and ICAM-1 on neutrophils in RSV bronchiolitis. Am J Physiol 275(5):L983-L989. https://doi.org/10.1152/ajplu ng.1998.275.5.1983

Wang JH, Kwas C, Wu L (2009a) Intercellular adhesion molecule 1 (ICAM-1), but not ICAM-2 and -3, is important for dendritic cell-mediated human immunodeficiency virus type 1 transmission. J Virol 83(9):4195-4204. https://doi.org/10.1128/ jvi.00006-09

Wang Y, Yan J, Shi Y, Li P, Liu C, Ma Q, Yang R, Wang X, Yang X, Cao C (2009b) Lack of association between polymorphisms of MASP2 and susceptibility to SARS coronavirus infection. BMC Infect Dis 9:51. https://doi.org/10.1186/1471-2334-9-51

Wang J, Li M, Wang H, Pan Y (2012) Identification of essential proteins based on edge clustering coefficient. IEEE/ACM Trans Comput Biol Bioinform 9(4):1070-1080. https://doi. org/10.1109/tcbb.2011.147

Wang J, Liu Y, Xie L, Li S, Qin X (2016a) Association of IL-17A and IL-17F gene polymorphisms with chronic hepatitis B and hepatitis B virus-related liver cirrhosis in a Chinese population: a casecontrol study. Clin Res Hepatol Gastroenterol 40(3):288-296. https://doi.org/10.1016/j.clinre.2015.10.004

Wang X, Ma K, Chen M, Ko KH, Zheng BJ, Lu L (2016b) IL-17A promotes pulmonary B-1a cell differentiation via induction of blimp-1 expression during influenza virus infection. PLoS Pathog 12(1):e1005367. https://doi.org/10.1371/journal.ppat.10053 67

Wang SM, Huang KJ, Wang CT (2019) Severe acute respiratory syndrome coronavirus spike protein counteracts BST2-mediated restriction of virus-like particle release. J Med Virol 91(10):1743-1750. https://doi.org/10.1002/jmv.25518

Warby TJ, Crowe SM, Jaworowski A (2003) Human immunodeficiency virus type 1 infection inhibits granulocyte-macrophage colonystimulating factor-induced activation of STAT5A in human monocyte-derived macrophages. J Virol 77(23):12630-12638. https://doi.org/10.1128/jvi.77.23.12630-12638.2003

Wasityastuti W, Yano Y, Ratnasari N, Triyono T, Triwikatmani C, Indrarti F, Heriyanto DS, Yamani LN, Liang Y, Utsumi T et al (2016) Protective effects of HLA-DPA1/DPB1 variants against Hepatitis B virus infection in an Indonesian population. Infect Genet Evol 41:177-184. https://doi.org/10.1016/j.meegi d.2016.03.034

Wei W, Guo H, Chang J, Yu Y, Liu G, Zhang N, Willard SH, Zheng S, Yu XF (2016) ICAM-5/telencephalin is a functional entry receptor for enterovirus D68. Cell Host Microbe 20(5):631-641. https ://doi.org/10.1016/j.chom.2016.09.013

Winkler CW, Woods TA, Rosenke R, Scott DP, Best SM, Peterson KE (2017) Sexual and Vertical Transmission of Zika Virus in anti-interferon receptor-treated Rag1-deficient mice. Sci Rep. 7(1):7176. https://doi.org/10.1038/s41598-017-07099-7

Wu D, Yang XO (2020) TH17 responses in cytokine storm of COVID19: an emerging target of JAK2 inhibitor Fedratinib. J Microbiol Immunol Infect S1684-1182(20):30065-30067. https://doi. org/10.1016/j.jmii.2020.03.005

Wu H, Haist V, Baumgärtner W, Schughart K (2010) Sustained viral load and late death in Rag2-/- mice after influenza A virus infection. Virol J. 7:172. https://doi.org/10.1186/1743-422x-7-172

Xiao F, Zuo Z, Cai G, Kang S, Gao X, Li T (2009) miRecords: an integrated resource for microRNA-target interactions. Nucleic Acids Res 37(Database Issue):D105-D110. https://doi.org/10.1093/nar/ gkn851

Xu R, Bao C, Huang H, Lin F, Yuan Y, Wang S, Jin L, Yang T, Shi $\mathrm{M}$, Zhang $\mathrm{Z}$ et al (2016) Low expression of CXCR1/2 on neutrophils predicts poor survival in patients with hepatitis B virusrelated acute-on-chronic liver failure. Sci Rep 6:38714. https:// doi.org/10.1038/srep38714

Xu X, Li Y, Liang Y, Yin M, Zhang Y, Huang L, Yu Z, Ni J (2018) Low responsiveness to a hepatitis $\mathrm{B}$ virus vaccine in a Chinese population lacks association with ITGAL, CD58, TNFSF15, CCL15, TGFB3, and BCL6 gene variants. Infect Genet Evol 64:126-130. https://doi.org/10.1016/j.meegid.2018.06.010 
Yang P, Li QJ, Feng Y, Zhang Y, Markowitz GJ, Ning S, Deng Y, Zhao J, Jiang S, Yuan Y et al (2012) TGF- $\beta$-miR-34a-CCL22 signaling-induced Treg cell recruitment promotes venous metastases of HBV-positive hepatocellular carcinoma. Cancer Cell 22(3):291-303. https://doi.org/10.1016/j.ccr.2012.07.023

Zaki N, Efimov D, Berengueres J (2013) Protein complex detection using interaction reliability assessment and weighted clustering coefficient. BMC Bioinformatics 14:163. https://doi. org/10.1186/1471-2105-14-163

Zehender G, Meroni L, De Maddalena C, Varchetta S, Monti G, Galli M (1997) Detection of hepatitis C virus RNA in CD19 peripheral blood mononuclear cells of chronically infected patients. J Infect Dis 176(5):1209-1214. https://doi.org/10.1086/514114

Zhang H, Penninger JM, Li Y, Zhong N, Slutsky AS (2020) Angiotensin-converting enzyme 2 (ACE2) as a SARS-CoV-2 receptor: molecular mechanisms and potential therapeutic target. Intensive Care Med 46(4):586-590. https://doi.org/10.1007/s00134-02005985-9

Zhao X, Song X, Bai X, Fei N, Huang Y, Zhao Z, Du Q, Zhang H, Zhang L, Tong D (2016) miR-27b attenuates apoptosis induced by transmissible gastroenteritis virus (TGEV) infection via targeting runt-related transcription factor 1 (RUNX1). PeerJ 4:e1635. https://doi.org/10.7717/peerj.1635

Zhao J, Zhu M, Jiang H, Shen S, Su X, Shi Y (2019) Combination of sphingosinE - 1-phosphate receptor 1 (S1PR1) agonist and antiviral drug: a potential therapy against pathogenic influenza virus. Sci Rep 9(1):5272. https://doi.org/10.1038/s41598-019-41760-7

Zheng J, Yang P, Tang Y, Zhao D (2015) A respiratory syncytial virus persistent-infected cell line system reveals the involvement of
SOCS1 in the innate antiviral response. Virol Sin 30(3):190-199. https://doi.org/10.1007/s12250-015-3597-0

Zhivaki D, Lemoine S, Lim A, Morva A, Vidalain PO, Schandene L, Casartelli N, Rameix-Welti MA, Hervé PL, Dériaud E et al (2017) Respiratory syncytial virus infects regulatory B cells in human neonates via chemokine receptor CX3CR1 and promotes lung disease severity. Immunity 46(2):301-314. https:// doi.org/10.1016/j.immuni.2017.01.010

Zhou G, Soufan O, Ewald J, Hancock REW, Basu N, Xia J (2019) NetworkAnalyst 3.0: a visual analytics platform for comprehensive gene expression profiling and meta-analysis. Nucleic Acids Res. https://doi.org/10.1093/nar/gkz240

Zhu DY, Jiang LF, Deng XZ, Xiao W, Pei JP, Li BJ, Wang CJ, Zhang JH, Zhang Q, Zhou ZX et al (2015) TBX21 polymorphisms are associated with virus persistence in hepatitis $C$ virus infection patients from a high-risk Chinese population. Eur J Clin Microbiol Infect Dis 34(7):1309-1318. https://doi.org/10.1007/s1009 6-015-2337-6

Zou L, Ruan F, Huang M, Liang L, Huang H, Hong Z, Yu J, Kang M, Song Y, Xia J et al (2020) SARS-CoV-2 Viral Load in Upper Respiratory Specimens of Infected Patients. N Engl J Med 382(12):1177-1179. https://doi.org/10.1056/nejmc2001737

Zuckerman E, Zuckerman T, Sahar D, Streichman S, Attias D, Sabo E, Yeshurun D, Rowe JM (2001) bcl-2 and immunoglobulin gene rearrangement in patients with hepatitis $\mathrm{C}$ virus infection. Br J Haematol 112(2):364-369. https://doi.org/10.104 6/j.1365-2141.2001.02573.x 